OPEN ACCESS

Edited by:

Kathleen S. Rockland

Boston University, United States

Reviewed by:

Henry Evrard,

Center for Integrative Neuroscience \&

Max Planck Institute, Germany

Song-Lin Ding,

Allen Institute for Brain Science,

United States

*Correspondence:

Martin Parent

martin.parent@fmed.ulaval.ca

Received: 09 November 2020

Accepted: 24 May 2021

Published: 13 August 2021

Citation:

Coulombe V, Saikali S, Goetz L, Takech MA, Philippe É, Parent A and Parent M (2021) A Topographic Atlas

of the Human Brainstem in the Ponto-Mesencephalic Junction Plane.

Front. Neuroanat. 15:627656.

doi: 10.3389/fnana.2021.627656

\section{A Topographic Atlas of the Human Brainstem in the Ponto-Mesencephalic Junction Plane}

\author{
Vincent Coulombe ${ }^{1}$, Stephan Saikali ${ }^{2}$, Laurent Goetz ${ }^{3}$, Mohamad A. Takech ${ }^{4}$, \\ Éric Philippe ${ }^{4}$, André Parent ${ }^{1}$ and Martin Parent ${ }^{1 *}$
}

${ }^{1}$ CERVO Brain Research Center, Quebec City, QC, Canada, ${ }^{2}$ Hôpital De L'Enfant-Jésus, CHU de Québec-Université Laval, Quebec City, QC, Canada, ${ }^{3}$ Hôpital Fondation Rothschild, Neurochirurgie pédiatrique - Unité Parkinson, Paris, France,

${ }^{4}$ Laboratoire d'Anatomie, Université Laval, Quebec City, QC, Canada

The human brainstem harbors neuronal aggregates that ensure the maintenance of several vital functions. It also acts as a major relay structure for the neuronal information that travels between the cerebral cortex, the cerebellum and the spinal cord. As such, this relatively small portion of the human brain houses a multitude of ascending and descending fibers that course among numerous nuclei whose exact boundaries are still uncertain. Such a large number of nuclei and fiber tracts confined to a relatively small and compact brain region imposes upon the brainstem a highly complex cytoarchitectonic organization that still needs to be deciphered. The present work provides a topographic atlas of the human brainstem composed of 45 anatomical plates, each containing a pair of adjacent sections stained with Cresyl Violet and Luxol Fast Blue to help delineating brainstem nuclei and fiber tracts, respectively. The plates, which cover the entire midbrain, pons and medulla oblongata, are composed of equally-spaced sections referenced and aligned parallel to the ponto-mesencephalic junction rather than the fastigium or the obex. This topographic landmark is particularly suitable for neurosurgical interventions aiming at specific nuclei of the mesencephalic tegmentum. In complement, we provide 8 anatomical plates containing adjacent sections stained for choline acetyltransferase and Luxol Fast Blue, taken through the midbrain and the pons. This open access atlas of the human brainstem is intended to assist neuroanatomists, neurosurgeons and neuropathologists in their work.

Keywords: midbrain, pons, medulla oblongata, cytoarchitecture, reticular formation, neurosurgery, neuropathology

\section{INTRODUCTION}

The human brainstem plays a crucial role in the maintenance of vital functions, such as respiratory and cardiovascular activities. Furthermore, it acts as a major relay station between the cerebral cortex, the cerebellum and the spinal cord, as first suggested by the English neurologist Thomas Willis more than 350 years ago (Willis, 1664). Yet, despite its prime importance in the coordination of several basic central nervous system activities, the brainstem is one of the least understood parts of the human brain. 
The organizational complexity of the brainstem relies, at least in part, from the fact that its houses a multitude of ascending and descending fiber tracts that course among a large number of nuclei whose exact boundaries are still a matter of controversy. Such a large number of nuclei and fiber tracts restricted to a relatively small brain region [the brainstem occupies $<3 \%$ of the total brain volume (Erbagci et al., 2012)] imposes upon the brainstem a highly complex cytoarchitectonic organization that still poses problems to neuroanatomists, neuropathologists, neurosurgeons, and imaging specialists.

Based on its external appearance along the rostrocaudal axis, the brainstem has traditionally been divided into the midbrain, the pons and the medulla oblongata. However, since the recent discovery of rhomobomeric segmentation based on developmental gene expression, the pons and medulla oblongata are now often referred together as the hindbrain, with the isthmus as its first segment (Watson et al., 2019; Paxinos et al., 2020). Along the dorsoventral axis, the brainstem can be divided into three distinctive parts: (1) a roof plate dorsal to the ventricular system known as the tectum, (2) a central core of cells and fibers beneath the ventricular system known as the tegmentum, and (3) a massive collection of ventrally located fibers derived from the cerebral cortex. The roof plate of the midbrain is represented by the tectum or quadrigeminal plate, consisting of the superior and inferior colliculi. At hindbrain levels, the roof plate is more elaborated and comprises the cerebellum and the tela choroidea, which will not be considered in detail here. The tegmentum of the midbrain and hindbrain contains the brainstem reticular formation or reticular core: a large collection of diffusely distributed cells closely intermingled with fibers that subserve multiple functions, and several more precisely delineated nuclei. The cortically derived ventral fiber system forms the crus cerebri at midbrain levels, one of the principal constituents of the ventral or basilar region at pontine levels, and the pyramids at medullary levels (Parent, 1996).

Some nuclei of the brainstem tegmentum are enriched in dopaminergic, cholinergic, serotoninergic or noradrenergic neurons known to be involved in the control of motor behavior, sleep and waking cycles, as well as various autonomic functions (Parent, 1996). Over the years, many efforts have been made to delineate brainstem nuclei and fiber tracts (Kolliker, 1896; Ziehen, 1903; Jacobsohn, 1909; Riley, 1943). Olszewski and Baxter were the first to provide a comprehensive and detailed description of the cytoarchitecture of the human brainstem illustrated with high-quality photomicrographs and outlined drawings of histological sections taken transversely to the longitudinal axis of the brainstem (Olszewski and Baxter, 1954). Despite the high quality of this work, the fact that no stereotaxic coordinates were provided has led many neurosurgeons to rely on the stereotaxic atlas of the human brain published by Schaltenbrand and Wahren (1977) for their interventions, with the inherent constraint of histological sections being oriented perpendicular to the anterior (ac) and posterior commissural (pc) plane. This coordinate system originally proposed by Talairach (Talairach et al., 1957; Talairach and Szikla, 1967; Talairach and Tournoux, 1988) is not ideally suited for brainstem stereotaxy because landmarks used for stereotaxic coordinates are too distant from regions of interest (Zrinzo et al., 2008; Goetz et al., 2019). The Allen Human Brain Atlas, which uses this sectioning plane, presents an overall view of brainstem structures in relation with the entire human brain by means of Nissl cell stain combined with parvalbumin and SMI-32 immunohistochemical markers (Ding et al., 2016). Efforts have also been deployed to provide brainstem stereotaxic atlases referenced on brainstem landmarks. Afshar et al. (1978), among others, have presented stereotaxic references for the human brainstem and cerebellar nuclei based on the floor of the fourth ventricle, the midline and a plane passing perpendicular to the floor of the fourth ventricle at the level of the fastigium. The stereotaxic atlas of Paxinos and Huang (1995) contains 64 histological sections perpendicular to the long axis of the brainstem, stained for Cresyl Violet and acetylcholine esterase as well as associated diagrams with delineated nuclei and fiber tracts. In their revised version (Paxinos et al., 2020), 159 plates are presented, 31 of which being from a different brain and used to delineate fiber tracts. In this atlas, sections of the brainstem were numbered based on their distance from the obex. More recently, numerous human brainstem descriptions from MRI have been published (Naidich et al., 2007; Deistung et al., 2013; Bianciardi et al., 2015; Tang et al., 2018; Rushmore et al., 2020). Among these, the Duvernoy's non-stereotaxic atlas of the human brainstem correlates transverse histological brainstem sections with corresponding clinical 3T and 9.4T MRI (Naidich et al., 2007) and is still widely used. The recent work of Rushmore and collaborators offers a detailed map of the human brainstem based on MRI dataset composed of 50-micron isotropic voxels from a post-mortem human brain (Rushmore et al., 2020).

In more recent studies (Ferraye et al., 2010; Goetz et al., 2019), the use of a new coordinate system referenced upon the ponto-mesencephalic junction (PMJ, a line that connects the inferior aspect of the quadrigeminal plate posteriorly with the foramen caecum of the interpeduncular fossa anteriorly), the floor of the fourth ventricle and the lateral mesencephalic sulci has been suggested to be more suitable for brainstem stereotaxy. This coordinate system, which refers to mesencephalic landmarks rather than the fastigium or the obex, has been adopted by some research groups (Thevathasan et al., 2011, 2012; Insola et al., 2012). It appears to be particularly suitable for neurosurgical interventions in the mesencephalic reticular formation, mainly because the references used are closer to neurosurgical areas of interest and easy to identify from MRI (Zrinzo et al., 2008). Readers looking for a color atlas of brainstem surgery should refer to the work of Spetzler and collaborators (Spetzler et al., 2017).

Our long-term interest in the basic aspects of the pathogenesis of various neurodegenerative diseases for which patients often present brainstem anomalies or are candidate for brainstem surgical interventions in the case of Parkinson's disease, has led us to produce a topographic atlas of the human brainstem composed of 45 anatomical plates, each containing a pair of adjacent sections stained with Cresyl Violet and Luxol Fast Blue used to delineate brainstem nuclei and fiber tracts, respectively. The plates cover the midbrain, the pons and the medulla oblongata and are composed of transverse sections taken parallel 
to the PMJ. In complement, we provide eight anatomical plates containing adjacent sections stained for choline acetyltransferase (ChAT) and Luxol Fast Blue taken through the midbrain and the pons. We hope that this open access atlas of the human brainstem will complement the work of our predecessors while providing an additional tool for fundamental and clinical research.

\section{METHOD}

\section{Specimen Preparation}

The post-mortem material was gathered from the brain of a 61-year-old woman who died from a pluri-metastatic colorectal cancer with no signs of neurological, neurodegenerative or psychiatric diseases. After a post-mortem delay of $5.5 \mathrm{~h}$, the brain was perfused through the internal carotids and the vertebral arteries with $4 \mathrm{~L}$ of cold saline solution ( $\mathrm{NaCL} 0.9 \%$ to which 20 units/mL of heparin was added), followed by $6 \mathrm{~L}$ of a cold fixative solution containing $4 \%$ paraformaldehyde (PFA) diluted in phosphate buffer $(\mathrm{PB}, 0.1 \mathrm{M}, \mathrm{pH} 7.4)$ and $4 \mathrm{~L}$ of $4 \%$ PFA to which $0.3 \%$ of glutaraldehyde was added. We then performed a MRI scan of the head with a 3T Philips Achieva (TE: $17 \mathrm{~ms}$; TR:4000 ms; slice thickness: $500 \mu \mathrm{m}$; duration $8 \mathrm{~h}$, see Supplementary Material) before brain extraction. The extracted brain weighed 1,328 g (Figures 1A-F). The brainstem was dissected out and post-fixed by immersion in $4 \%$ PFA for 4 days. Tissue samples were obtained from the Human Anatomy Laboratory at Université Laval and kept in the Human Brain Bank of the CERVO research Center. Both Institutions required informed consent before donation of tissues. The Ethics Committee at Université Laval approved the brain collecting procedures, as well as the storage and handling of post-mortem human brain tissues.

The brainstem was dissected and cut along a sagittal plane at $1 \mathrm{~mm}$ from the midline (Figure 1G). From the right hemibrainstem that contained the midline, four different blocks were cut along the ponto-mesencephalic junction plane (PMJ), a line that connects the inferior aspect of the quadrigeminal plate (qpc) posteriorly with the foramen caecum $(\mathrm{Fc})$ of the interpeduncular fossa (ipf) anteriorly (Figure 1H). The PMJ is depicted by a red line on the different plates. Its exact position can be found on Figure $1 \mathbf{H}$ and on plate $10(0.00 \mathrm{~mm})$. We estimate that there exists a difference of 10 degrees between the PMJ plane and the ac-pc plane (Figure 1H) and 20 degrees between the PMJ plane and the one used in the atlas of Paxinos (Paxinos et al., 2020). Each of the blocks were then cut at $4^{\circ} \mathrm{C}$ into $50 \mu \mathrm{m}$-thick sections using a vibratome (Leica VT1200S). The sections were all collected serially in phosphate buffer saline (PBS, 0.1 M, pH 7.4). Pairs of adjacent sections were selected out of 22 sections and then processed for Cresyl Violet and Luxol Fast Blue, respectively, providing a mean interval of $1.1 \mathrm{~mm}$ between each plate. Because some sections were damaged during the cutting process, in some cases, we decided to choose the next undamaged section instead of the adjacent one, for a maximum of $400 \mu \mathrm{m}$ interval between Cresyl Violet and Luxol Fast Blue stained sections. Because losses of tissue inevitably occurred between each block, in addition to the known section thickness and intervals, we referred to the MRI

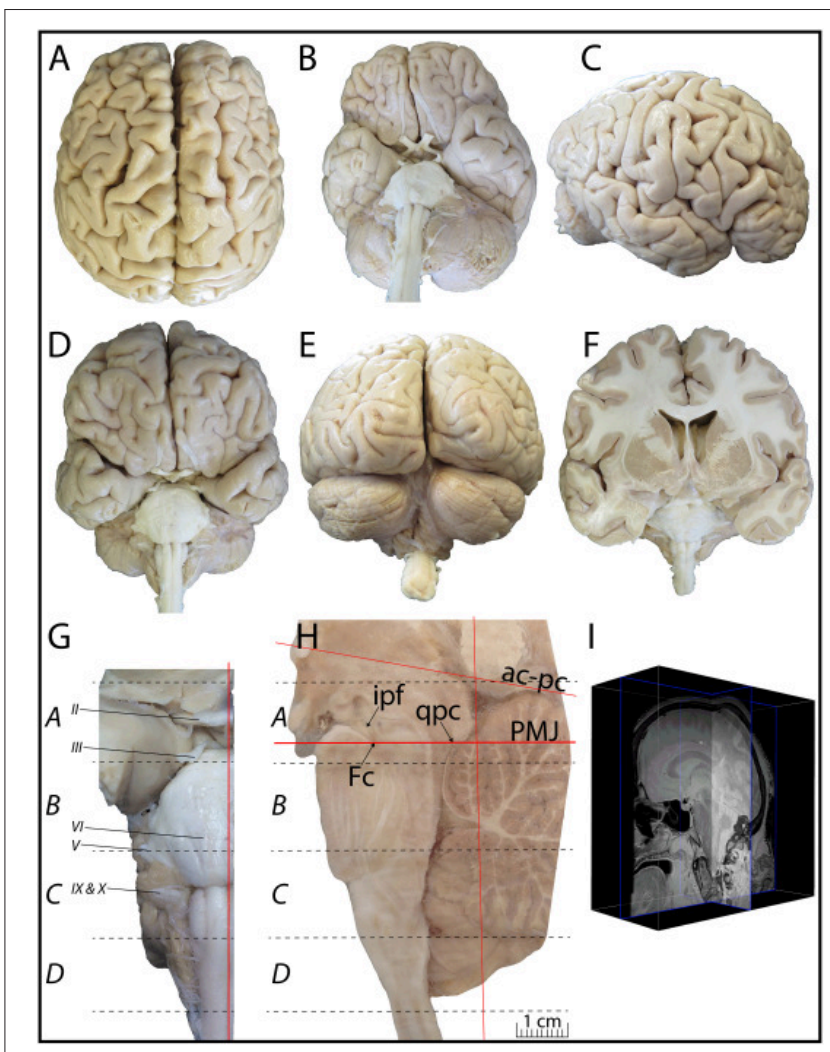

FIGURE 1 | (A-E) Superior (A), inferior (B), lateral (C), anterior (D), and posterior (E) views of the whole brain used. This brain comes from a generous donation of a 61-year-old woman who died from a pluri-metastatic colorectal cancer with no signs of neurological, neurodegenerative or psychiatric diseases. The perfused and extracted brain weighed 1,328 g. (F) Coronal view of the sectioned brain. (G) Picture of the dissected brainstem cut through its longitudinal axis, along a sagittal plane, at $1 \mathrm{~mm}$ from the midline. $\mathbf{( H )}$ From the right hemi-brainstem containing the midline, four blocks were cut along the ponto-mesencephalic junction plane (PMJ in $\mathbf{H}$ ). Block A extends from 11.19 to $-0.98 \mathrm{~mm}$ (plates $1-11$ ), block B from -3.87 to $-21.33 \mathrm{~mm}$ (plates $12-24$ ), block C from -24.09 to $-36.87 \mathrm{~mm}$ (plates 25-38), and block D from -39.70 to $-51.70 \mathrm{~mm}$ (plates $39-45$ ), relative to the PMJ. (I) Brain MRI is available as Supplementary Material.

in order to confirm the exact position of each section, relative to the PMJ (Figure 1I).

\section{Cresyl Violet Staining}

Sections intended for Cresyl Violet staining were first mounted on gelatin-coated microscope slides, air dried at room temperature and incubated overnight in 95\% ethanol at $56^{\circ} \mathrm{C}$. The slides were then rinsed 15 times in distilled water and immersed for $3 \mathrm{~min}$ at room temperature in a pre-heated and filtered solution of Cresyl Violet acetate (catalog no. C5042, Sigma, St-Louis, MO, USA) dissolved at $0.1 \%$ in distilled water. Sections were then rinsed in distilled water followed by 10 times in ethanol 95\%, two times in a solution of ethanol/acid acetic $0.5 \%$, five times in ethanol $95 \%$. The rinses in ethanol $95 \%$ and ethanol/acid acetic were repeated until the desired staining intensity was obtained. Sections were dehydrated in ethanol and 
coverslipped with Permount (Permount Mounting Medium, catalog no. SP15, Thermo Fisher Scientific, Waltman, MA, USA).

\section{Luxol Fast Blue Staining}

Sections intended for Luxol Fast Blue staining were first mounted on gelatin-coated microscope slides, air dried at room temperature and rinsed 10 times in ethanol $95 \%$. They were then incubated overnight at $56^{\circ} \mathrm{C}$ in Luxol Fast Blue (Solvent Blue 38, catalog no. S3382, Sigma, St-Louis, MO, USA) dissolved at $0.1 \%$ in ethanol. They were rinsed once in ethanol 95\%, 10 times in lithium carbonate $(0.01 \%)$ and 10 times in ethanol $70 \%$. When needed, rinses in lithium carbonate $(0.01-0.1 \%)$ and ethanol were repeated until the desired staining intensity was obtained. Sections were dehydrated in ethanol and coverslipped with Permount. To increase contrast, five sections stained for Luxol Fast Blue had to be also stained with Cresyl Violet (Plates 9, 10, $26,31,37)$.

\section{Choline Acetyltransferase Immunostaining}

Eight additional equally-spaced sections taken trough the midbrain and the pons (between $+4.88 \mathrm{~mm}$ and $-10.59 \mathrm{~mm}$ ) were stained for choline acetyltransferase (ChAT), the enzyme responsible for the synthesis of acetylcholine (plates A-H). After three rinses in PBS, the sections were placed for $20 \mathrm{~min}$ at room temperature in hydrogen peroxide $\left(3 \% \mathrm{H}_{2} \mathrm{O}_{2}\right.$ diluted in ethanol) to eliminate endogenous peroxidase activity. The free-floating sections were then placed in sodium borohydride $(0.5 \%$ diluted in PBS) for $30 \mathrm{~min}$. After three rinses in PBS, the sections were preincubated for $1 \mathrm{~h}$ at room temperature in a PBS solution containing $2 \%$ normal rabbit serum and $0.5 \%$ Triton $\mathrm{X}-100$, and incubated $48 \mathrm{~h}$ at $4{ }^{\circ} \mathrm{C}$ in the same solution to which the goat anti-ChAT antibody was added (catalog no. AB144P, EMD Milipore Corporation, Billerica, MA, 1:20). The sections were then rinsed, reincubated for $1 \mathrm{~h}$ at room temperature with a rabbit anti-goat biotinylated IgG (catalog \# BA-5000; Vector Labs, Burlingame, CA, USA; 1:200). After three rinses in PBS, the sections were reincubated for $1 \mathrm{~h}$ at room temperature in $2 \%$ avidin-biotin-peroxidase complex (catalog \# PK-4000; Vector Labs). The bound peroxidase was revealed by placing the sections in a medium containing 0.05\% 3,3'-diaminobenzidine tetrahydrochloride (DAB, catalog \#D5637; Sigma) and 0.005\% $\mathrm{H}_{2} \mathrm{O}_{2}$ in $0.05 \mathrm{M}$ Tris buffer, $\mathrm{pH}$ 7.6. The reaction was stopped after $8 \mathrm{~min}$ by several washes in Tris buffer and PBS. Immunostained sections were mounted on gelatine-coated slides, dehydrated in ethanol and coverslipped with Permount.

Cresyl Violet, Luxol Fast Blue and ChAT-stained sections were digitalized at $1200 \mathrm{dpi}$ (pixel resolution of $1 \mu \mathrm{m}$ ) using a slide scanner (TISSUEscopeTM 4000, Huron Technologies, Waterloo, Ontario, Canada) equipped with a 10X objective.

\section{Methodological Considerations and Limitations}

Chemical fixation of the brain and section processing inevitably lead to shrinkage. In order to minimize shrinkage, blocks were cut at $4^{\circ} \mathrm{C}$ with a vibratome, precluding the need of cryoprotection in sucrose solution and freezing. A comparison of the size of the red nucleus as it appears on post-mortem brain MRI images and on Cresyl Violet stained sections, has revealed the shrinkage to be of the order of $4 \%$. No pre-mortem MRI was available to assess shrinkage that might have been caused by the perfusion. It should be reminded that this atlas is based on a single brain of a 61year-old woman and that existing inter-individual variations should carefully be taken into account using provided MRI (see Supplementary Material). Three different magnifications had to be used to provide plates presenting histological sections of adequate size (plates 1-6, plates 7-29, and plates 30-45). Therefore, readers should pay close attention to individual scale bars when comparisons are made between plates. Segmentation of brainstem nuclei and fiber tracts from Cresyl Violet and Luxol Fast Blue stained sections were performed following careful examination of histological sections, so as to avoid arbitrary delineation. Only structures that could be easily delineated in our preparations are identified and dashed lines are used when brainstem regions don't show clear histological boundaries. For example, the cuneiform nucleus $(\mathrm{CnF})$ and the tegmental part of pontine reticular nucleus (PnTn) are broadly delineated with dashed lines because their boundaries with surrounding structures could not be clearly established. Therefore, the CnF, as defined in the present study, might comprise a portion of the mesencephalic reticular formation, whereas the PnTn could include parts of the isthmic reticular formation, the retroisthmic nucleus, the retrorubral field and the ventrolateral tegmental nucleus, as defined by Paxinos (Paxinos et al., 2020). Our segmentation was confirmed with the help of other human brainstem atlases (Olszewski and Baxter, 1954; Schaltenbrand and Wahren, 1977; Afshar et al., 1978; Paxinos and Huang, 1995; Naidich et al., 2007; Paxinos et al., 2020). Readers might refer to other human brainstem atlases for more extensive delineation of brainstem subnuclei and additional plates, in different sectioning planes. The nomenclature and abbreviations used in the present study are largely based on those of Paxinos and Watson (1982). For brainstem nuclei that didn't show clear subdivisions, we used single abbreviations to identify structures composed of several subnuclei. For example, the abbreviation $\mathrm{Sp} 5$ for spinal trigeminal nucleus, as delineated in the present atlas, includes the oral (Sp5O), interpolar (Sp5I), and caudal (Sp5C) parts of the nucleus, and the abbreviation $\mathrm{VC}$ for ventral cochlear nucleus includes the anterior (VCA) and posterior (VCP) parts of the nucleus that are delineated in other atlases. Likewise, the abbreviation PAG for periaqueductal gray refers to all PAG columns. A complete list of abbreviations and equivalent Latin names, as published in Terminologia Anatomica (Terminology, 1998) is also provided. The asterisks added to some abbreviations indicate that these abbreviations are slightly different from those used in the human brainstem atlas of Paxinos (Paxinos et al., 2020) or point to structures that are not identified in that atlas. 
LIST OF ABBREVIATIONS

\begin{tabular}{|c|c|c|}
\hline $3 \mathrm{~N}$ & Oculomotor nucleus & Nucleus nervi oculomotorii \\
\hline $3 n$ & Oculomotor nerve & Nervus oculomotorius \\
\hline $4 \mathrm{~N}$ & Trochlear nucleus & Nucleus nervi trochlearis \\
\hline $4 n$ & Trochlear nerve & Nervus trochlearis \\
\hline $4 \mathrm{~V}$ & Fourth ventricle & Ventriculus quartus \\
\hline $5 \mathrm{ADi}$ & Motor trigeminal nucleus, anterior digastric part & Nucleus motorius nervi trigemini, pars digastrica anterior \\
\hline $5 \mathrm{~N}$ & Motor trigeminal nucleus & Nucleus motorius nervi trigemini \\
\hline $5 S p$ & Lamina 5 of the spinal gray & Lamina spinalis $V$ \\
\hline $5 \mathrm{Te}$ & Motor trigeminal nucleus, temporal part & Nucleus motorius nervi trigemini, pars temporalis \\
\hline $5 \operatorname{Tr}$ & Trigeminal transition zone & Zona transitionalis nervi trigemini \\
\hline $6 \mathrm{~N}$ & Abducens nucleus & Nucleus nervi abducentis \\
\hline $6 n$ & Abducens nerve & Nervus abducens \\
\hline 7DM & Facial nucleus, dorsomedial part & Nucleus nervi facialis, pars dorsomedialis \\
\hline $7 \mathrm{~N}$ & Facial nucleus & Nucleus nervi facialis \\
\hline $7 n$ & Facial nerve & Nervus facialis \\
\hline $7 \mathrm{SH}$ & Facial nucleus, stylohyoid part & Nucleus nervi facialis, pars stylohyoidalis \\
\hline $7 \mathrm{VL}$ & Facial nucleus, ventrolateral part & Nucleus nervi facialis, pars ventrolateralis \\
\hline $8 n$ & Vestibulocochlear nerve & Nervus vestibulocochlearus \\
\hline $9 n$ & Glossopharyngeal nerve & Nervus glossopharyngeus \\
\hline $9 S p$ & Lamina 9 of the spinal gray & Lamina spinalis IX \\
\hline $10 \mathrm{~N}$ & Dorsal motor nucleus of the vagus nerve & Nucleus dorsalis motorius nervi vagi \\
\hline $10 n$ & Vagus nerve & Nervus vagus \\
\hline $12 \mathrm{~N}$ & Hypoglossal nucleus & Nucleus nervi hypoglossi \\
\hline $12 n$ & Hypoglossal nerve & Nervus hypoglossus \\
\hline $\mathrm{ac}^{*}$ & Anterior commissure & Commissura anterior \\
\hline $\mathrm{al}^{*}$ & Ansa lenticularis & Ansa lenticularis \\
\hline Amb & Ambiguus nucleus & Nucleus ambiguous \\
\hline $\mathrm{Amg}^{*}$ & Amygdaloid complex & Corpus amygdaloideum \\
\hline $\mathrm{Aq}$ & Aqueduct & Aqueductus cerebri \\
\hline $\operatorname{Ar}$ & Arcuate nucleus & Nucleus arcuatus \\
\hline $\mathrm{BIC}$ & Nucleus of the brachium of the inferior colliculus & Nucleus brachium colliculi inferioris \\
\hline bic & Brachium of the inferior colliculus & Brachium colliculi inferioris \\
\hline CAT & Nucleus of the central acoustic tract & Nucleus centralis tractus acustica \\
\hline $\mathrm{CbH}^{*}$ & Cerebellar hemisphere & Hemispherium cerebelli \\
\hline $\mathrm{CbV}^{*}$ & Cerebellar vermis & Vermis cerebelli \\
\hline $\mathrm{CC}$ & Central canal & Canalis centralis \\
\hline$C d^{*}$ & Caudate nucleus & Nucleus caudatus \\
\hline $\mathrm{CG}^{*}$ & Central gray & Griseum centralis \\
\hline CLi & Caudal linear nucleus of the raphe & Nucleus raphes linearis, pars caudalis \\
\hline $\mathrm{CnF}$ & Cuneiform nucleus & Nucleus cuneiformis \\
\hline $\mathrm{cp}$ & Cerebral peduncle & Pedunculus cerebri \\
\hline csp & Corticospinal tract & Tractus corticospinalis \\
\hline Ct & Conterminal nucleus & Nucleus conterminalis \\
\hline $\operatorname{ctg}$ & Central tegmental tract & Tractus tegmentalis centralis \\
\hline cth $^{\star}$ & Cerebellothalamic tract & Tractus cerebellothalamicus \\
\hline $\mathrm{Cu}$ & Cuneate nucleus & Nucleus cuneatus \\
\hline $\mathrm{cu}$ & Cuneate fasciculus & Fasciculus cuneatus \\
\hline $\mathrm{DC}$ & Dorsal cochlear nucleus & Nucleus cochlearis dorsalis \\
\hline DLG & Dorsal lateral geniculate nucleus & Nucleus geniculatum laterale \\
\hline DLL & Dorsal nucleus of the lateral lemnicus & Nucleus dorsalis lemnisci lateralis \\
\hline DPGi & Dorsal paragigantocellular nucleus & Nucleus paragigantocellularis dorsalis \\
\hline DR & Dorsal raphe nucleus & Nucleus raphes dorsalis \\
\hline dsc & Dorsal spinocerebellar tract & Tractus spinocerebellaris dorsalis \\
\hline
\end{tabular}


LIST OF ABBREVIATIONS | Continued

\begin{tabular}{|c|c|c|}
\hline $\mathrm{DTg}^{*}$ & Dorsal tegmental nucleus & Nucleus tegmentalis dorsalis \\
\hline $\mathrm{ECu}$ & External cuneate nucleus & Nucleus cuneatus, pars externa \\
\hline emlt* $^{\star}$ & External medullary lamina of the thalamus & Lamina medullaris externa thalami \\
\hline EnR & Endorestiform nucleus & Nucleus endorestiformis \\
\hline EW & Edinher-Westphal nucleus & Nucleus accessorii nervi oculomotorii \\
\hline $\mathrm{FC}^{*}$ & Foramen caecum & Foramen caecum \\
\hline $\mathrm{ft}^{\star}$ & Thalamic fasciculus & Fasciculus thalamicus \\
\hline Gr & Gracile nucleus & Nucleus gracilis \\
\hline gr & Gracile fasciculus & Fasciculus gracilis \\
\hline 18 & Interstitial nucleus of the vestibular part of the 8th nerve & Nucleus interstitialis nervi vestibulocochlearus, pars vestibularis \\
\hline ia & Internal arcuate fibers & Fibrae arcuatae internae \\
\hline $1 C^{*}$ & Inferior colliculus & Colliculi inferioris \\
\hline $\mathrm{IOD}$ & Inferior olive, dorsal nucleus & Nucleus olivaris dorsalis \\
\hline $\mathrm{IOM}$ & Inferior olive, medial nucleus & Oliva inferior, nucleus medialis \\
\hline $\mathrm{IOPr}$ & Inferior olive, principal nucleus & Oliva inferior, nucleus principalis \\
\hline $\mathrm{IP}$ & Interpeduncular nucleus & Nucleus interpeduncularis \\
\hline ipf & Interpeduncular fossa & Fossa interpeduncularis \\
\hline $\mathrm{JxO}$ & Juxtaolivary nucleus & Oliva inferior, juxta nucleus \\
\hline LC & Locus coeruleus & Locus coeruleus \\
\hline Ics & Lateral corticospinal tract & Tractus corticospinalis lateralis \\
\hline LDTg & Laterodorsal tegmental nucleus & Nucleus tegmentalis laterodorsalis \\
\hline $\mathrm{Li}$ & Linear nucleus of the hindbrain & Nucleus linearis rhombencephali \\
\hline$\|$ & Lateral lemniscus & Lemniscus lateralis \\
\hline MB & Mammillary body & Corpus mammillare \\
\hline $\mathrm{mcp}$ & Middle cerebellar peduncle & Pedunculus cerebellaris medius \\
\hline $\mathrm{MdC}^{*}$ & Medullary reticular nucleus, central part & Formatio reticularis medulares, pars centralis \\
\hline $\mathrm{MdD}$ & Medullary reticular nucleus, dorsal part & Formatio reticularis medulares, pars dorsalis \\
\hline MaV & Medullary reticular nucleus, ventral part & Formatio reticularis medulares, pars ventralis \\
\hline Me5 & Mesencephalic trigeminal nucleus & Nucleus mesencephalicus nervi trigemini \\
\hline me5 & Mesencephalic trigeminal tract & Tractus mesencephalici nervi trigemini \\
\hline MG & Medial geniculate nucleus & Nucleus geniculatum mediale \\
\hline $\mathrm{ml}$ & Medial lemniscus & Lemniscus medialis \\
\hline mlf & Medial longitudinal fasciculus & Fasciculus Iongitudinalis medialis \\
\hline $\mathrm{MnR}$ & Median raphe nucleus & Nucleus raphes medianus \\
\hline MPB & Medial parabrachial nucleus & Nucleus parabrachialis medialis \\
\hline $\mathrm{MPCu}$ & Medial pericuneate nucleus & Nucleus pericuneatus medialis \\
\hline$m t^{*}$ & Mammillothalamic tract & Tractus mammillothalamicus \\
\hline MVe & Medial vestibular nucleus & Nucleus vestibularis medialis \\
\hline NB & Basal nucleus of Meynert & Nucleus basalis Meynerti \\
\hline oc & Olivocerebellar tract & Tractus olivocerebellaris \\
\hline opt & Optic tract & Tractus opticus \\
\hline
\end{tabular}


LIST OF ABBREVIATIONS | Continued

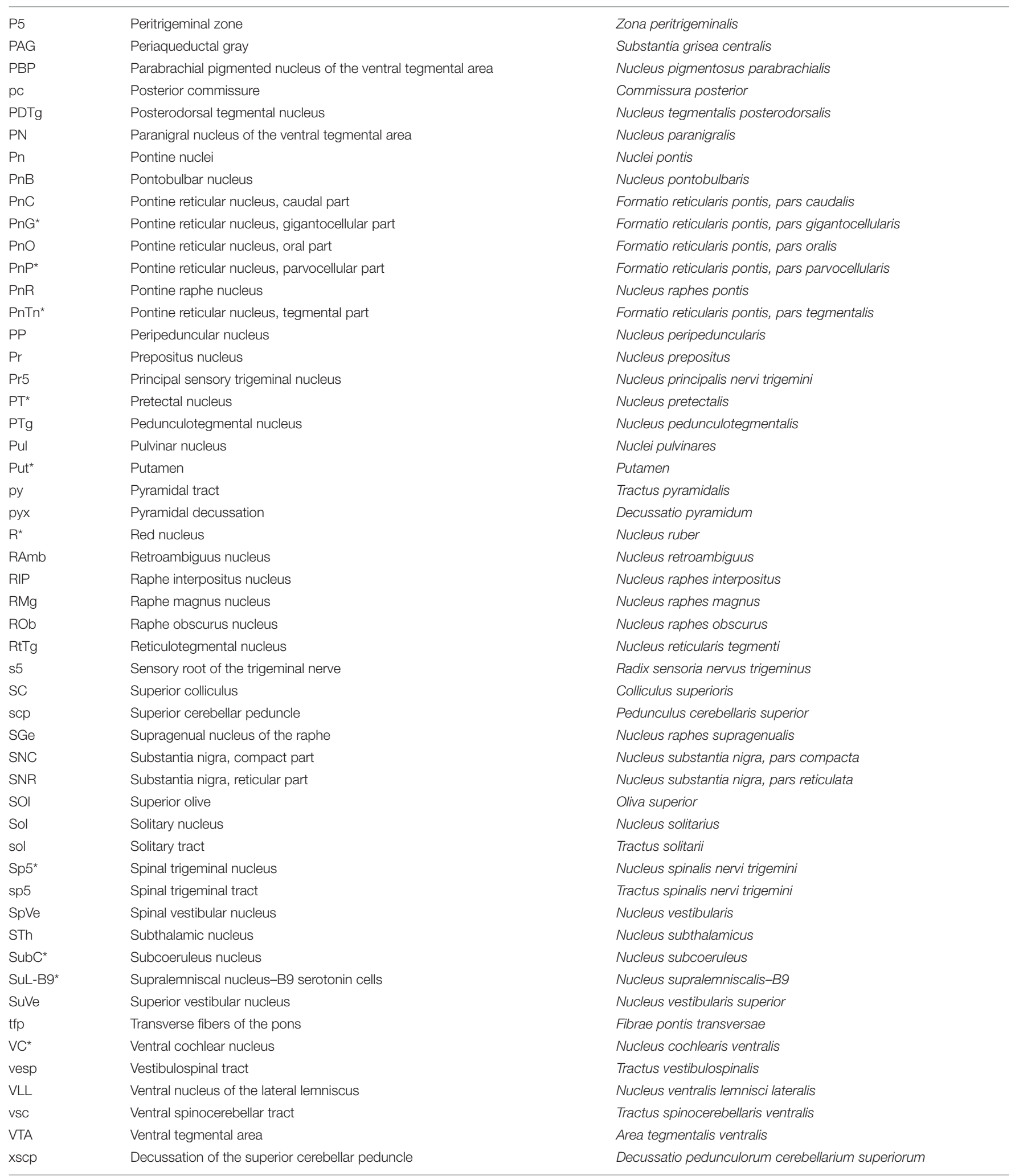

*Asterisks indicate abbreviations that are slightly different from those used in the human brainstem atlas of Paxinos et al. (2020) or point to structures that are not identified in that atlas. 


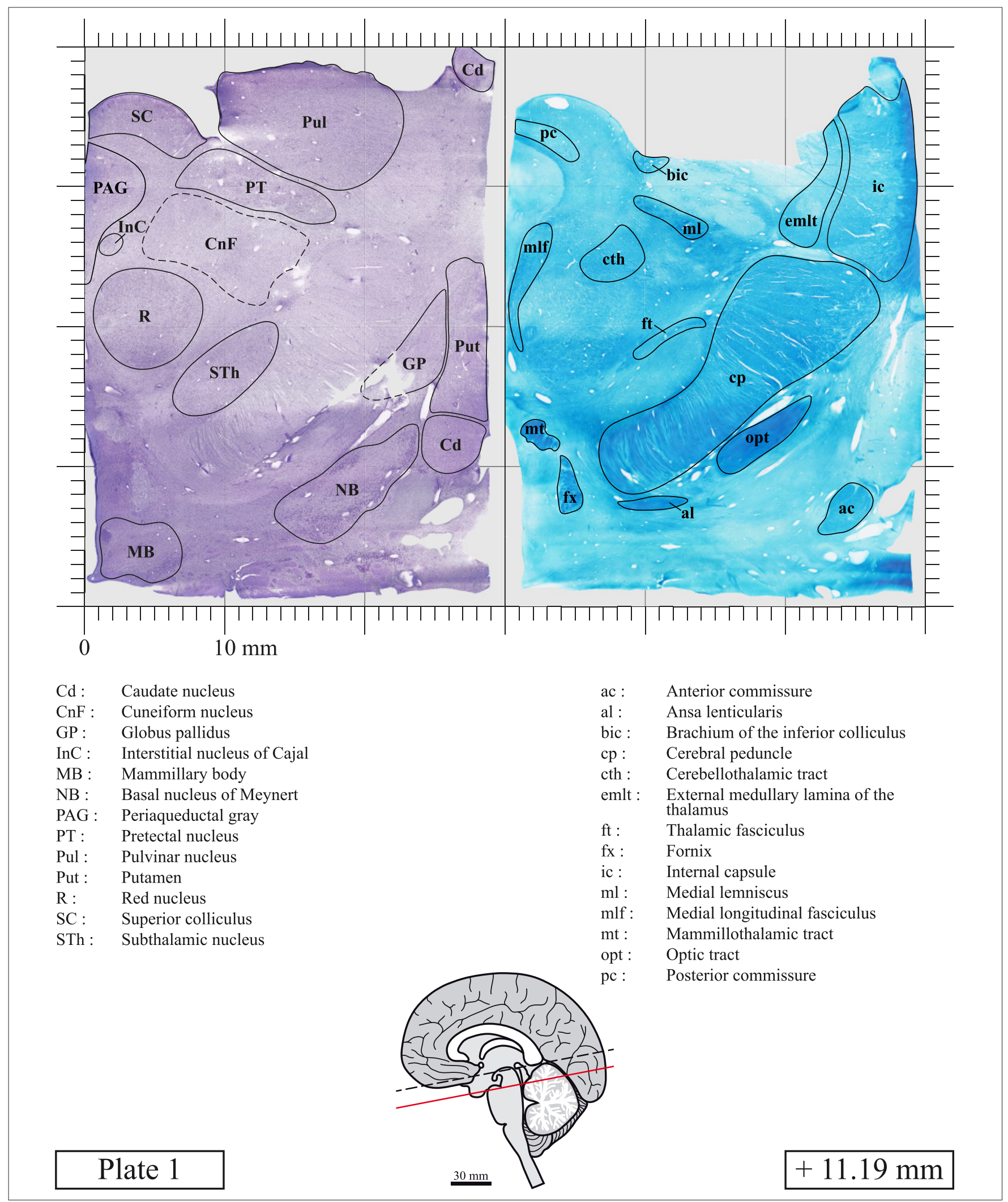




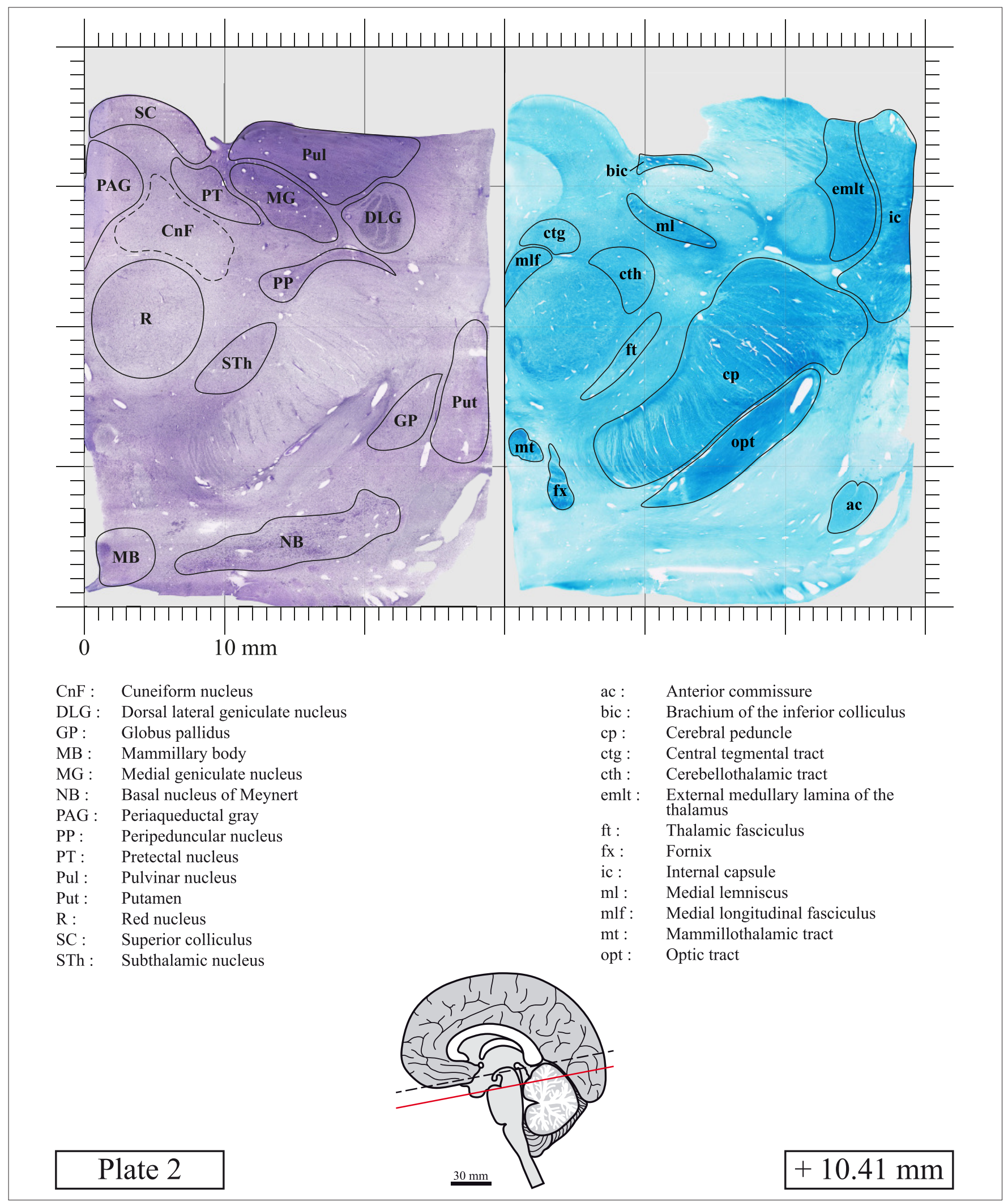




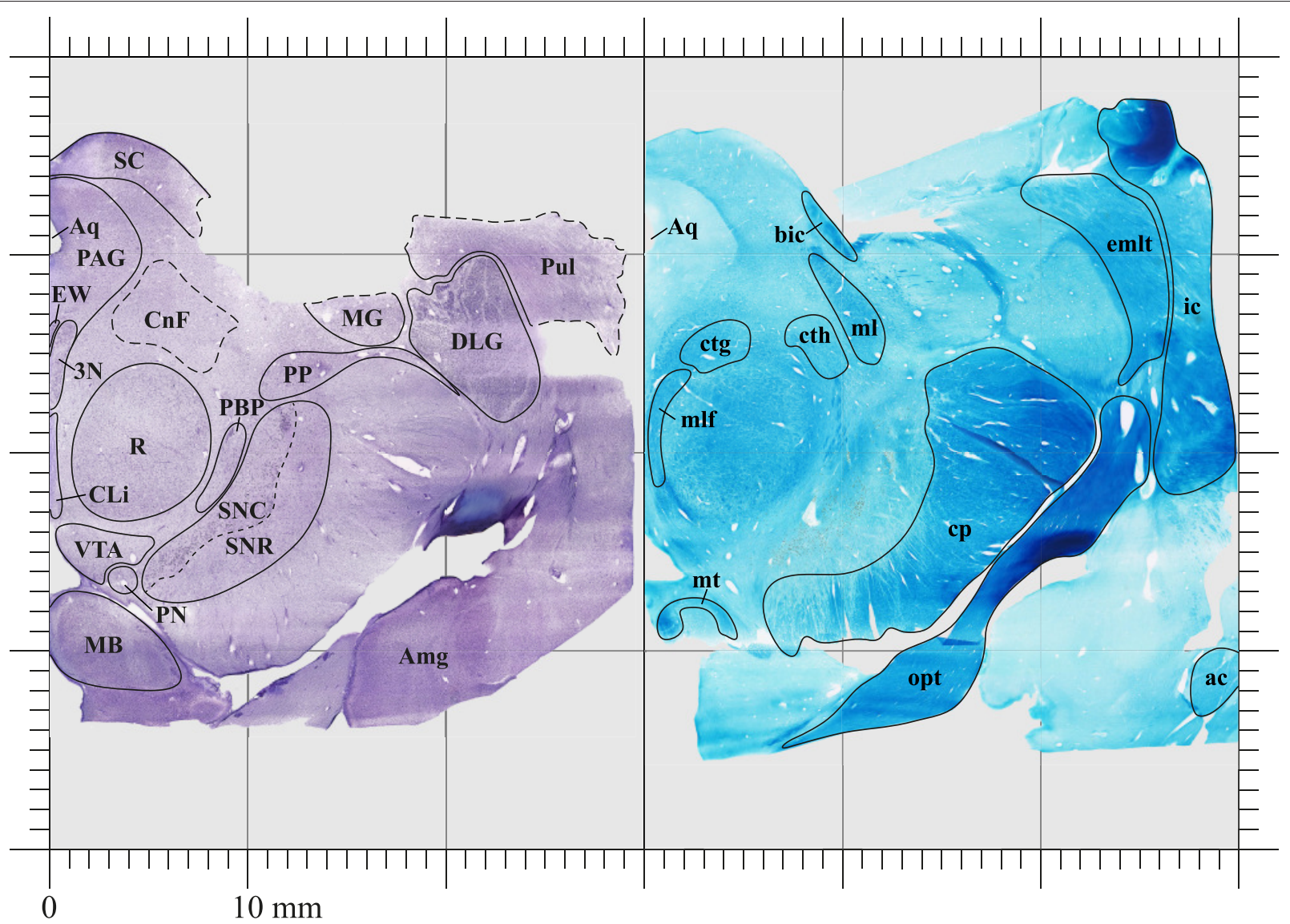

3N : Oculomotor nucleus

Amg : Amygdaloid complex

Aq : Aqueduct

CLi : Caudal linear nucleus of the raphe

$\mathrm{CnF}$ : Cuneiform nucleus

DLG : Dorsal lateral geniculate nucleus

EW : Edinher-Westphal nucleus

MB : Mammillary body

MG : Medial geniculate nucleus

PAG : Periaqueductal gray

PBP : Parabrachial pigmented nucleus of the ventral tegmental area

PN : $\quad$ Paranigral nucleus of the ventral tegmental area

PP : $\quad$ Peripeduncular nucleus

Pul : Pulvinar nucleus

$\mathrm{R}$ : $\quad$ Red nucleus

SC : $\quad$ Superior colliculus

SNC : Substantia nigra, compact part

SNR : Substantia nigra, reticular part

VTA : Ventral tegmental area ac : Anterior commissure

Aq : Aqueduct

bic : Brachium of the inferior colliculus

cp : Cerebral peduncle

ctg : Central tegmental tract

cth : Cerebellothalamic tract

emlt : External medullary lamina of the thalamus

ic : Internal capsule

$\mathrm{ml}$ : Medial lemniscus

mlf : Medial longitudinal fasciculus

$\mathrm{mt}$ : Mammillothalamic tract

opt : Optic tract

\section{Plate 3}

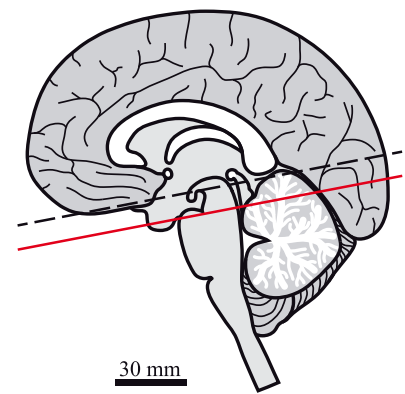




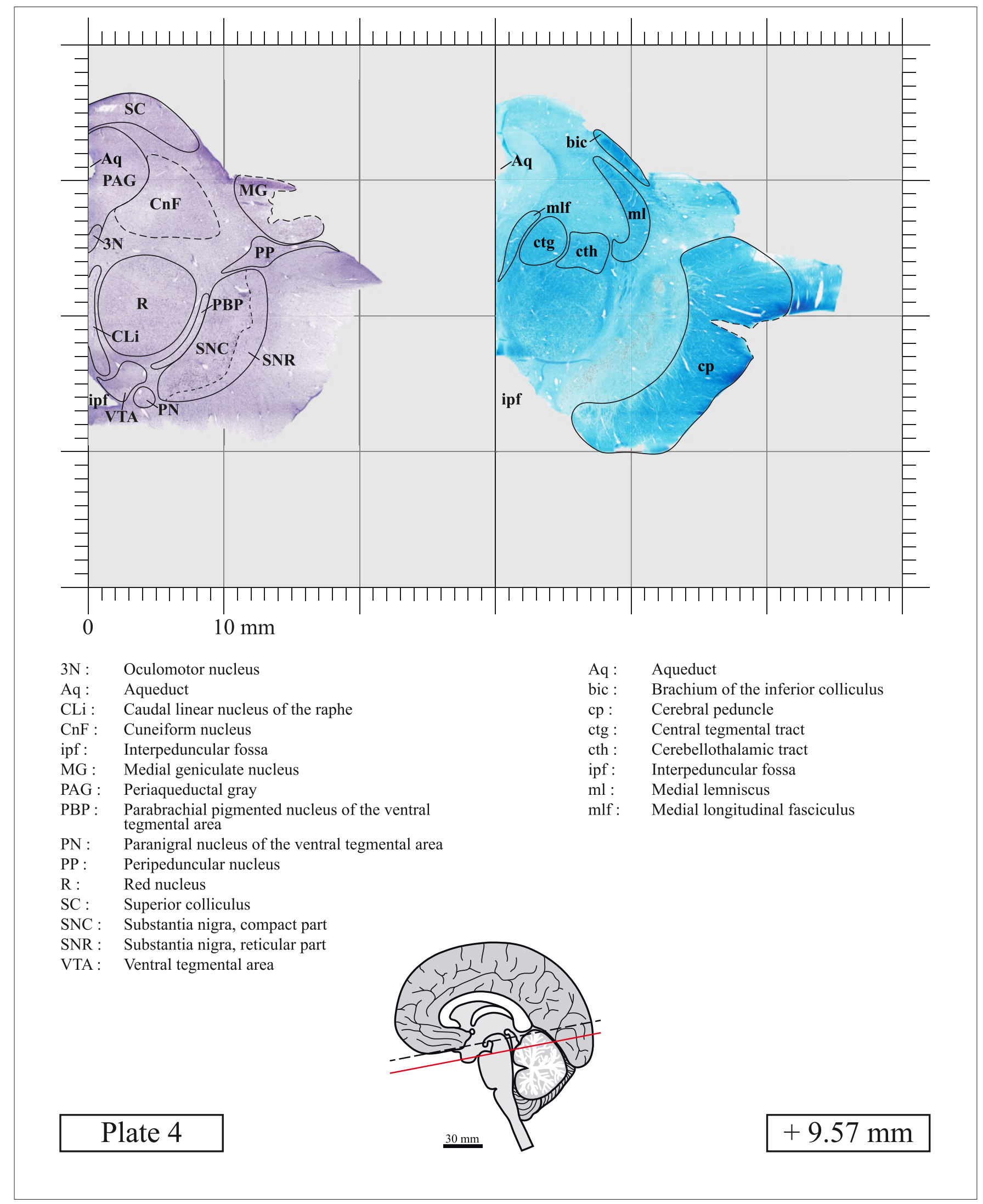




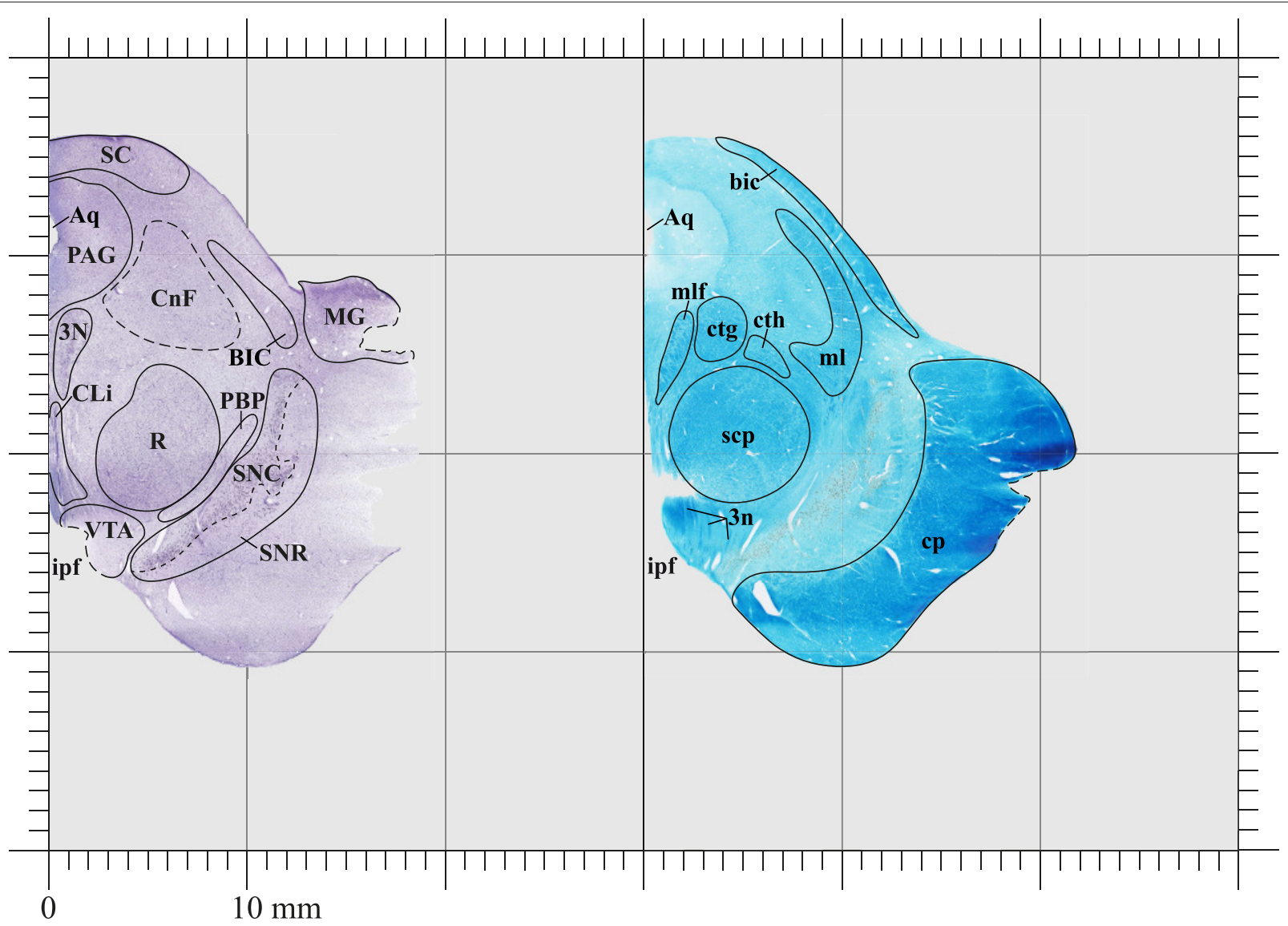

3N : Oculomotor nucleus

Aq : Aqueduct

BIC : Nucleus of the brachium of the inferior colliculus

CLi : Caudal linear nucleus of the raphe

$\mathrm{CnF}$ : Cuneiform nucleus

ipf : Interpeduncular fossa

MG : Medial geniculate nucleus

PAG : Periaqueductal gray

PBP : Parabrachial pigmented nucleus of the ventral tegmental area

$\mathrm{R}$ : $\quad$ Red nucleus

3n: $\quad$ Oculomotor nerve

Aq : Aqueduct

bic : Brachium of the inferior colliculus

cp : Cerebral peduncle

ctg : Central tegmental tract

cth : Cerebellothalamic tract

ipf : Interpeduncular fossa

$\mathrm{ml}$ : $\quad$ Medial lemniscus

mlf : Medial longitudinal fasciculus

scp : $\quad$ Superior cerebellar peduncle

SC : Superior colliculus

SNC : Substantia nigra, compact part

SNR : Substantia nigra, reticular part

VTA : Ventral tegmental area

\section{Plate 5}

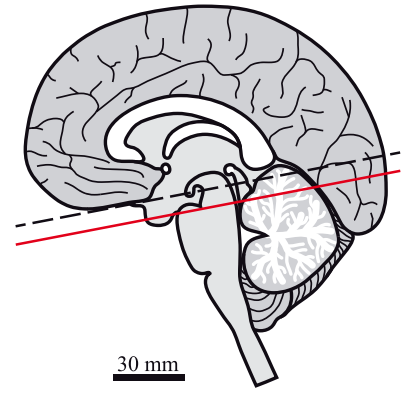




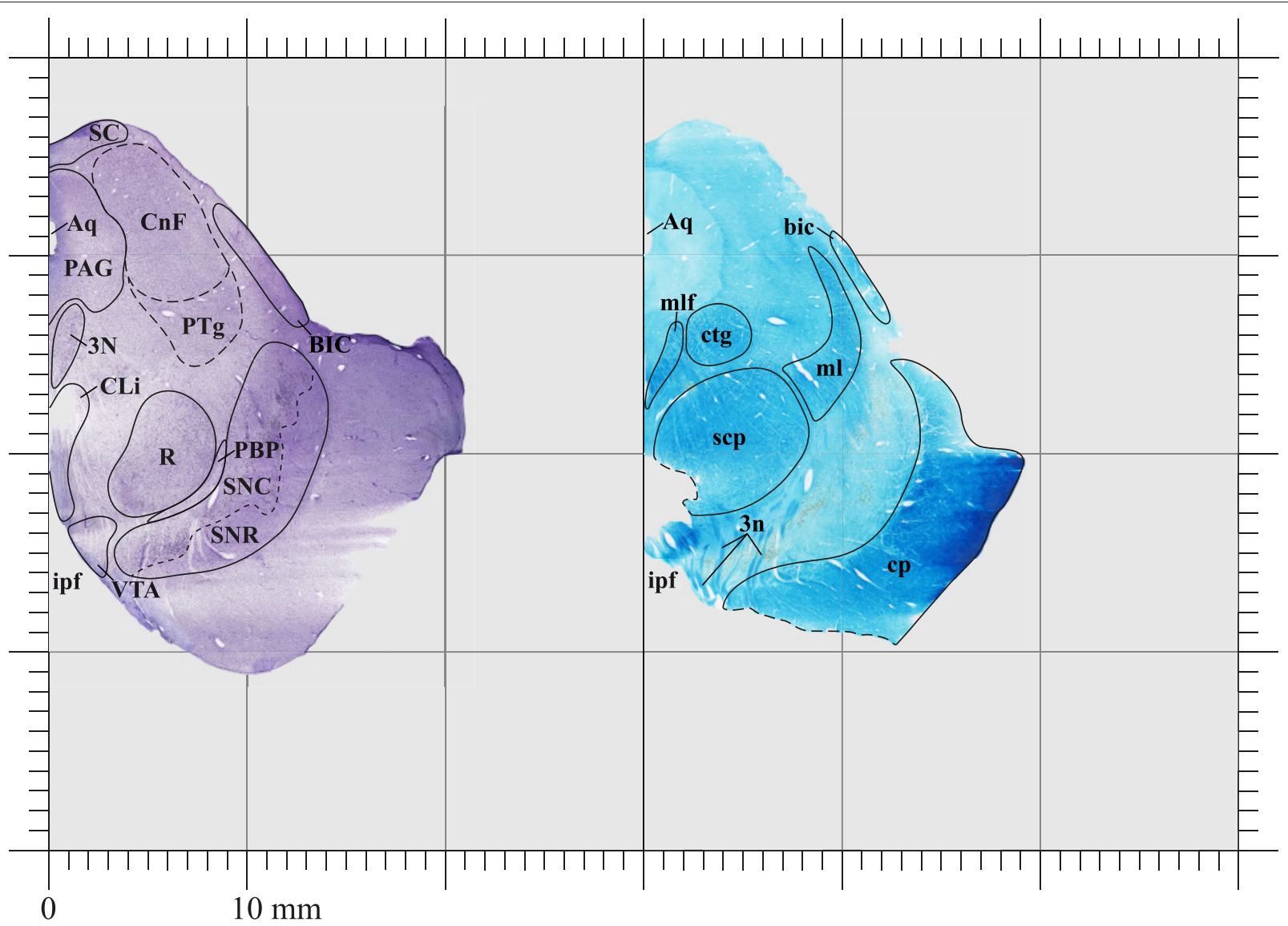

3N : Oculomotor nucleus

Aq : Aqueduct

BIC : Nucleus of the brachium of the inferior colliculus

CLi : Caudal linear nucleus of the raphe

$\mathrm{CnF}$ : Cuneiform nucleus

ipf : Interpeduncular fossa

PAG : Periaqueductal gray

PBP : Parabrachial pigmented nucleus of the ventral

PTg : $\quad \begin{aligned} & \text { tegmental area } \\ & \text { Pedunculotegmental nucleus }\end{aligned}$

3n: $\quad$ Oculomotor nerve

Aq : Aqueduct

bic : Brachium of the inferior colliculus

cp : Cerebral peduncle

ctg : Central tegmental tract

ipf : Interpeduncular fossa

$\mathrm{ml}$ : $\quad$ Medial lemniscus

mlf : Medial longitudinal fasciculus

scp : $\quad$ Superior cerebellar peduncle

$\mathrm{R}$ : $\quad$ Red nucleus

$\mathrm{SC}: \quad$ Superior colliculus

SNC : Substantia nigra, compact part

SNR : Substantia nigra, reticular part

VTA : Ventral tegmental area

\section{Plate 6}

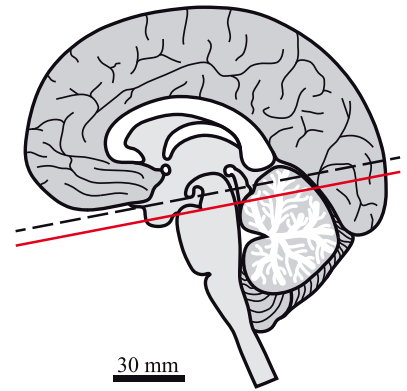




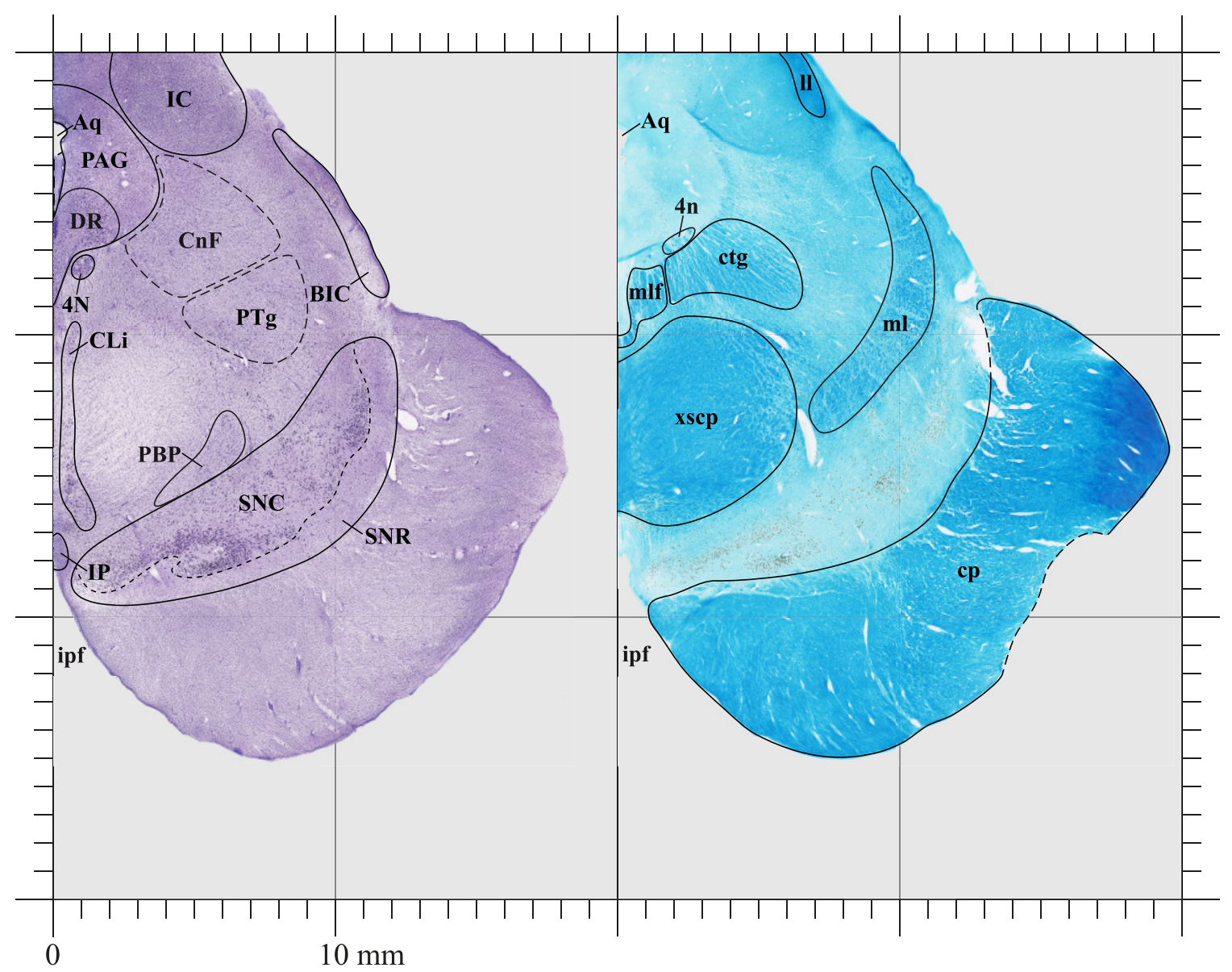

4N: $\quad$ Trochlear nucleus

Aq : Aqueduct

BIC : Nucleus of the brachium of the inferior colliculus

CLi : Caudal linear nucleus of the raphe

$\mathrm{CnF}$ : Cuneiform nucleus

DR : Dorsal raphe nucleus

IC : Inferior colliculus

IP : Interpeduncular nucleus

ipf : Interpeduncular fossa

PAG : Periaqueductal gray

PBP : Parabrachial pigmented nucleus of the ventral tegmental area

PTg : Pedunculotegmental nucleus

SNC : Substantia nigra, compact part

SNR : Substantia nigra, reticular part

\section{Plate 7}

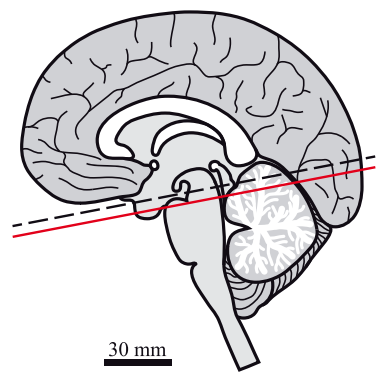

4n: Trochlear nerve

Aq : Aqueduct

cp : Cerebral peduncle

ctg : Central tegmental tract

ipf : Interpeduncular fossa

11: $\quad$ Lateral lemniscus

$\mathrm{ml}$ : $\quad$ Medial lemniscus

mlf: Medial longitudinal fasciculus

xscp : Decussation of the superior cerebellar peduncle 


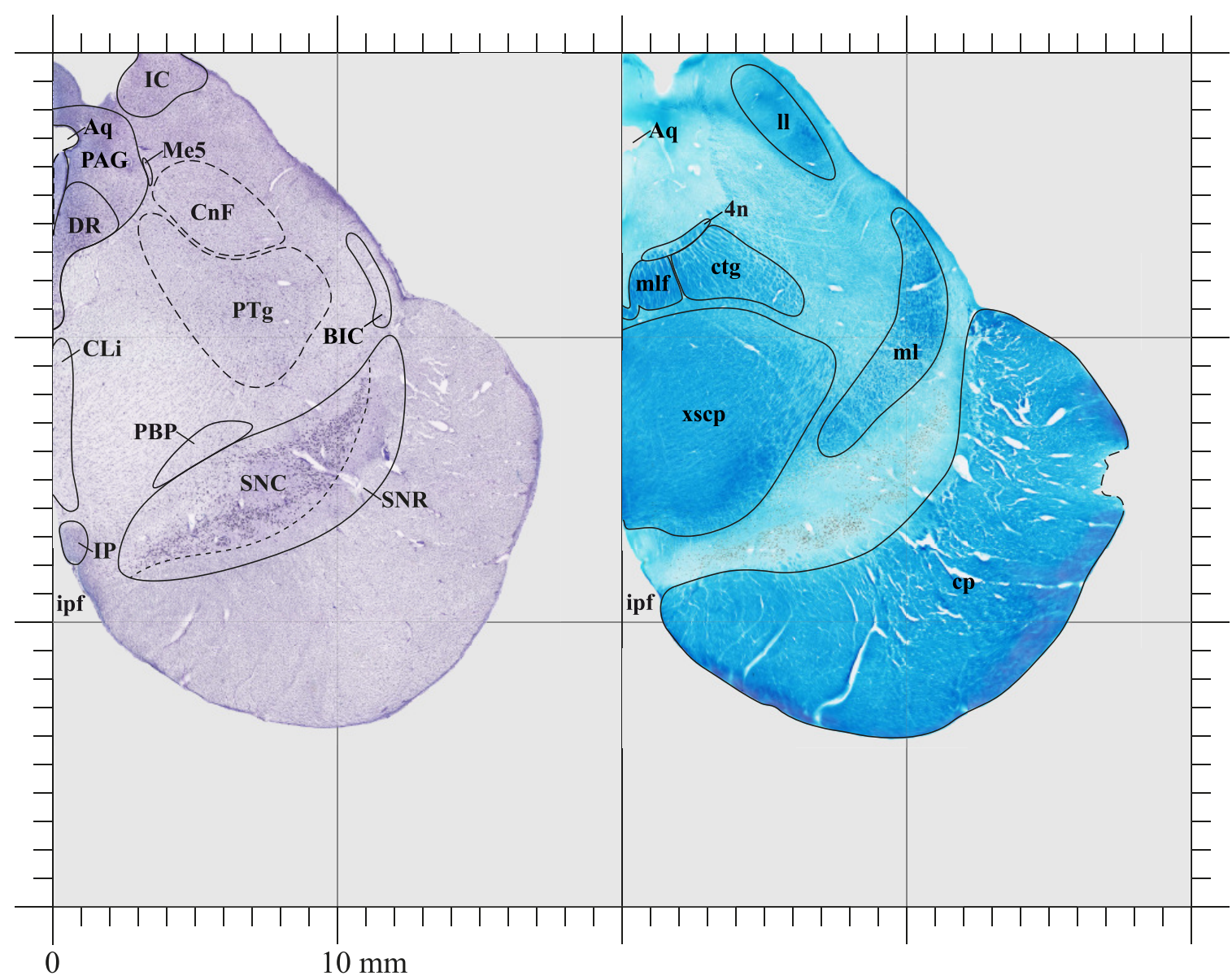

Aq : Aqueduct

BIC : Nucleus of the brachium of the inferior colliculus

CLi : Caudal linear nucleus of the raphe

$\mathrm{CnF}$ : Cuneiform nucleus

DR : Dorsal raphe nucleus

IC : Inferior colliculus

IP : Interpeduncular nucleus

ipf : Interpeduncular fossa

Me5: Mesencephalic trigeminal nucleus

PAG : Periaqueductal gray

PBP : Parabrachial pigmented nucleus of the ventral tegmental area

PTg : Pedunculotegmental nucleus

SNC : Substantia nigra, compact part

SNR : Substantia nigra, reticular part

\section{Plate 8}

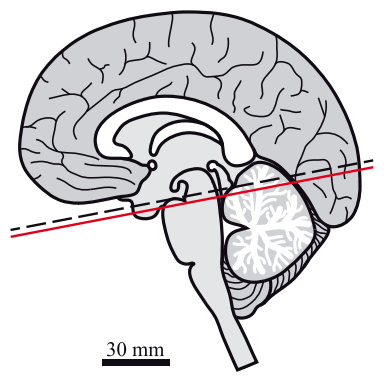

4n: $\quad$ Trochlear nerve

Aq : Aqueduct

cp : Cerebral peduncle

ctg : Central tegmental tract

ipf : Interpeduncular fossa

11: Lateral lemniscus

$\mathrm{ml}$ : $\quad$ Medial lemniscus

mlf: Medial longitudinal fasciculus

xscp : Decussation of the superior cerebellar peduncle 


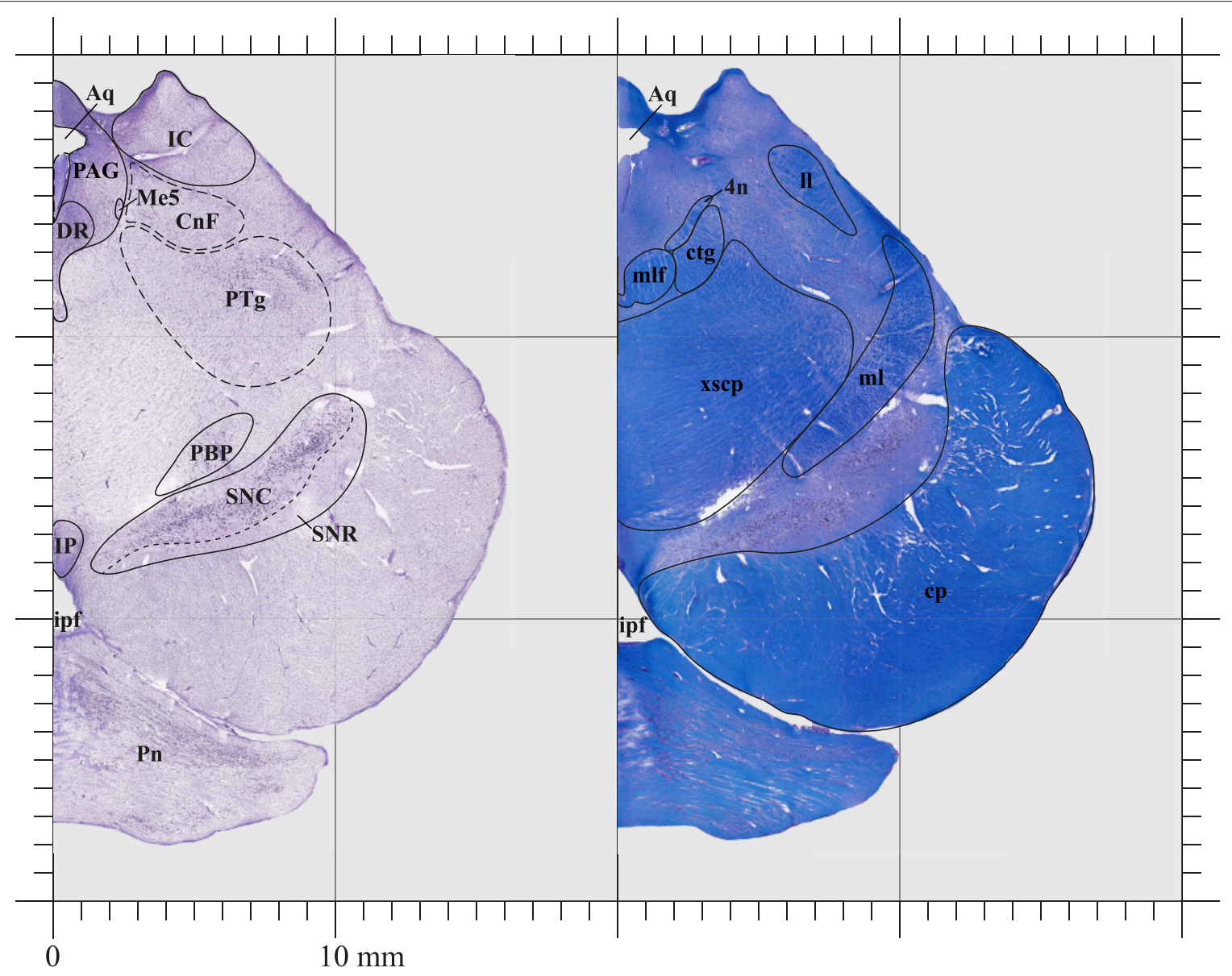

Aq : Aqueduct

CnF : Cuneiform nucleus

DR : Dorsal raphe nucleus

IC : Inferior colliculus

IP : Interpeduncular nucleus

ipf : Interpeduncular fossa

Me5: Mesencephalic trigeminal nucleus

PAG : Periaqueductal gray

PBP : Parabrachial pigmented nucleus of the ventral tegmental area

Pn : $\quad$ Pontine nuclei

PTg : Pedunculotegmental nucleus

SNC : Substantia nigra, compact part

SNR : Substantia nigra, reticular part 4n: Trochlear nerve

Aq : Aqueduct

cp : Cerebral peduncle

ctg : Central tegmental tract

ipf : Interpeduncular fossa

11 : $\quad$ Lateral lemniscus

$\mathrm{ml}$ : $\quad$ Medial lemniscus

mlf : Medial longitudinal fasciculus

xscp : Decussation of the superior cerebellar peduncle

\section{Plate 9}

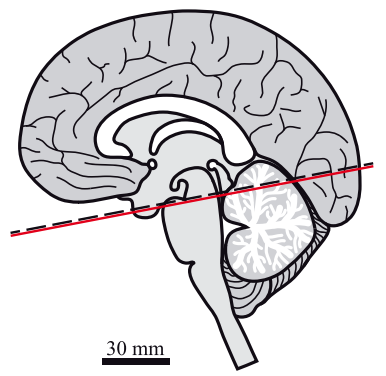

$+0.94 \mathrm{~mm}$ 


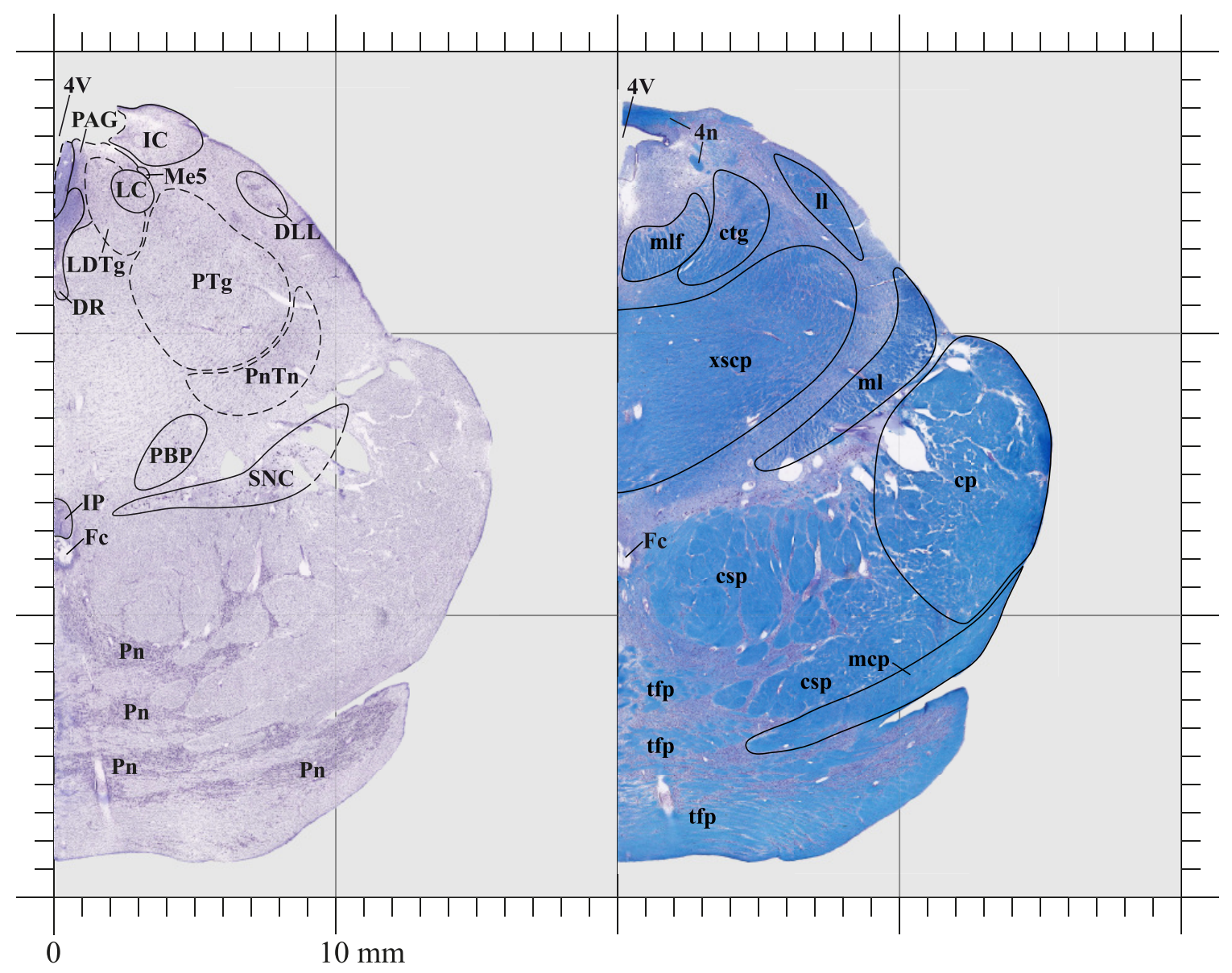

4V : Fourth ventricle

DLL : Dorsal nucleus of the lateral lemniscus

4n: Trochlear nerve

DR : Dorsal raphe nucleus

4V : $\quad$ Fourth ventricle

Fc : Foramen caecum

IC : Inferior colliculus

IP : Interpeduncular nucleus

LC : Locus coeruleus

LDTg : Laterodorsal tegmental nucleus

Me5: Mesencephalic trigeminal nucleus

PAG : Periaqueductal gray

PBP : Parabrachial pigmented nucleus of the ventral tegmental area

cp : $\quad$ Cerebral peduncle

csp : Corticospinal tract

ctg : Central tegmental tract

Fc : Foramen caecum

11: Lateral lemniscus

mcp : Middle cerebellar peduncle

$\mathrm{ml}$ : Medial lemniscus

mlf: Medial longitudinal fasciculus

tfp : $\quad$ Transverse fibers of the pons

Pn : $\quad$ Pontine nuclei

xscp : Decussation of the superior cerebellar peduncle

PnTn : Pontine reticular nucleus, tegmental part

PTg : Pedunculotegmental nucleus

SNC : Substantia nigra, compact part

Plate 10

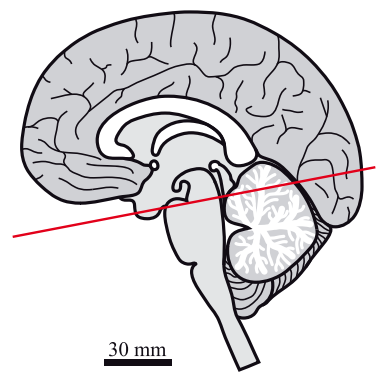

$+0.00 \mathrm{~mm}$ 


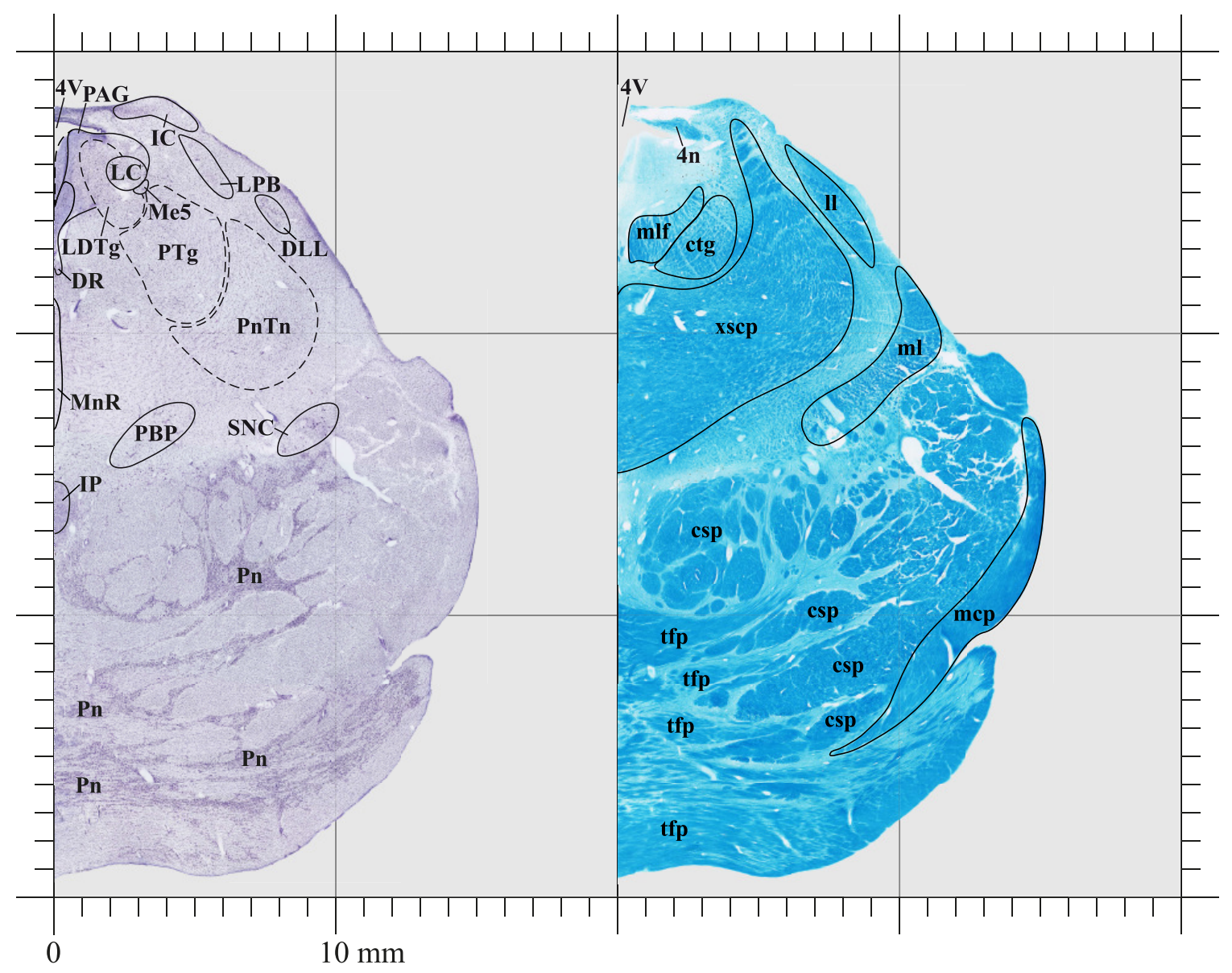

4V : Fourth ventricle

DLL: Dorsal nucleus of the lateral lemniscus

DR : Dorsal raphe nucleus

IC : Inferior colliculus

IP : Interpeduncular nucleus

LC : Locus coeruleus

LDTg : Laterodorsal tegmental nucleus

LPB : Lateral parabrachial nucleus

Me5: Mesencephalic trigeminal nucleus

MnR: Median raphe nucleus

PAG : Periaqueductal gray

PBP : Parabrachial pigmented nucleus of the ventral tegmental area

Pn : $\quad$ Pontine nuclei

PnTn : Pontine reticular nucleus, tegmental part

PTg: Pedunculotegmental nucleus

SNC : Substantia nigra, compact part

\section{Plate 11}

4n: Trochlear nerve

4V: $\quad$ Fourth ventricle

csp : Corticospinal tract

ctg : Central tegmental tract

11: Lateral lemniscus

mcp : Middle cerebellar peduncle

$\mathrm{ml}$ : Medial lemniscus

mlf: $\quad$ Medial longitudinal fasciculus

tfp : Transverse fibers of the pons

xscp : Decussation of the superior cerebellar peduncle 


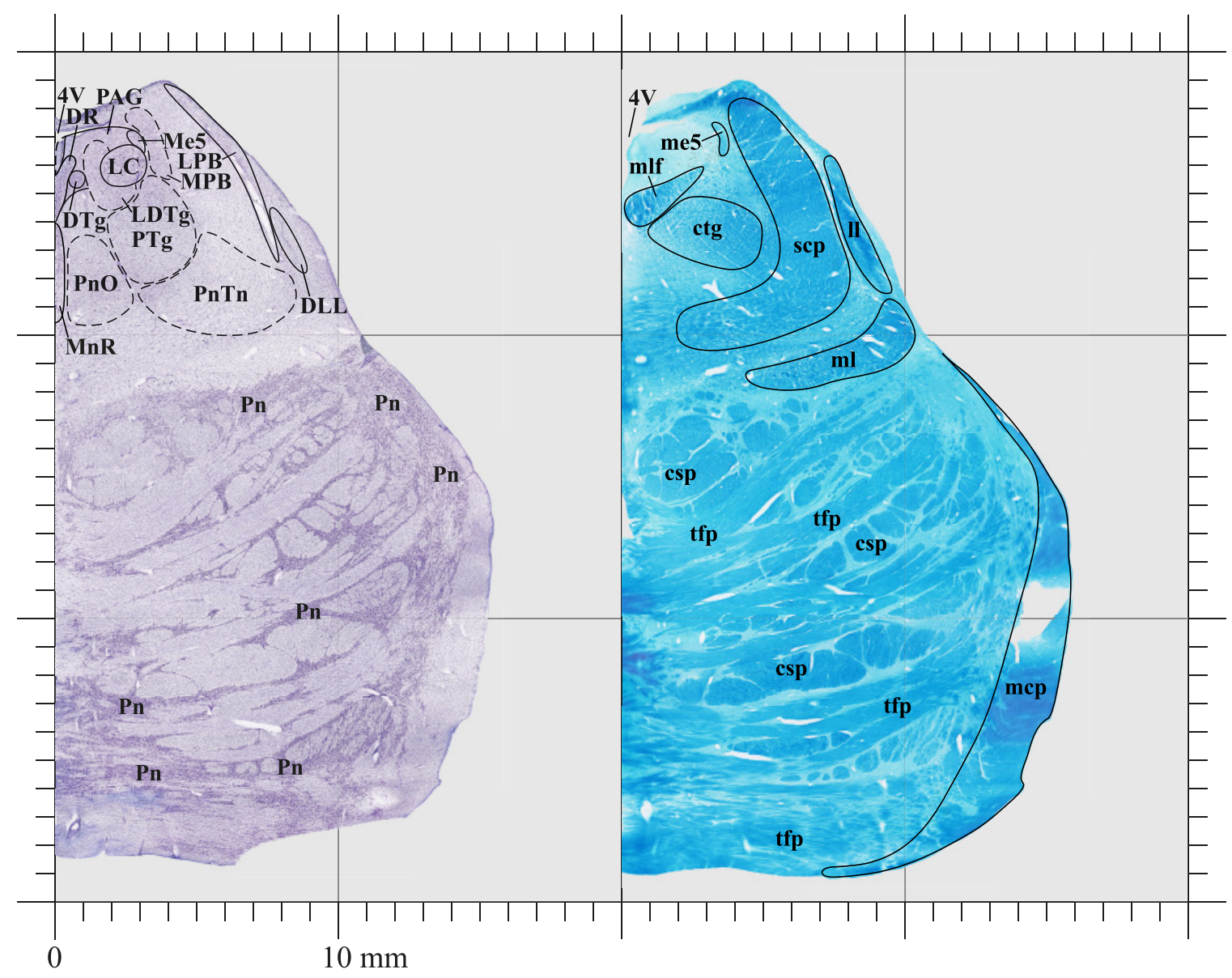

4V: $\quad$ Fourth ventricle

DLL : Dorsal nucleus of the lateral lemniscus

DR : Dorsal raphe nucleus

DTg : Dorsal tegmental nucleus

LC : Locus coeruleus

LDTg : Laterodorsal tegmental nucleus

LPB : Lateral parabrachial nucleus

Me5 : Mesencephalic trigeminal nucleus

MnR : Median raphe nucleus

MPB : Medial parabrachial nucleus

PAG : Periaqueductal gray

Pn : $\quad$ Pontine nuclei

PnO : Pontine reticular nucleus, oral part

PnTn: Pontine reticular nucleus, tegmental part

PTg : Pedunculotegmental nucleus

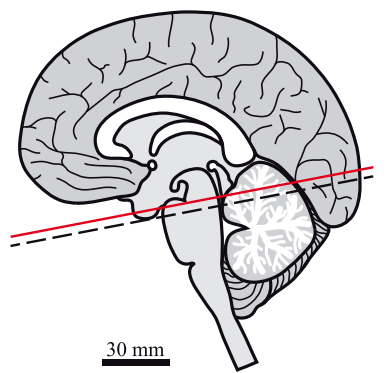

4V: $\quad$ Fourth ventricle

csp : Corticospinal tract

ctg : Central tegmental tract

11 : Lateral lemniscus

mcp : Middle cerebellar peduncle

me5 : Mesencephalic trigeminal tract

$\mathrm{ml}$ : $\quad$ Medial lemniscus

mlf: Medial longitudinal fasciculus

scp : $\quad$ Superior cerebellar peduncle

tfp : $\quad$ Transverse fibers of the pons 


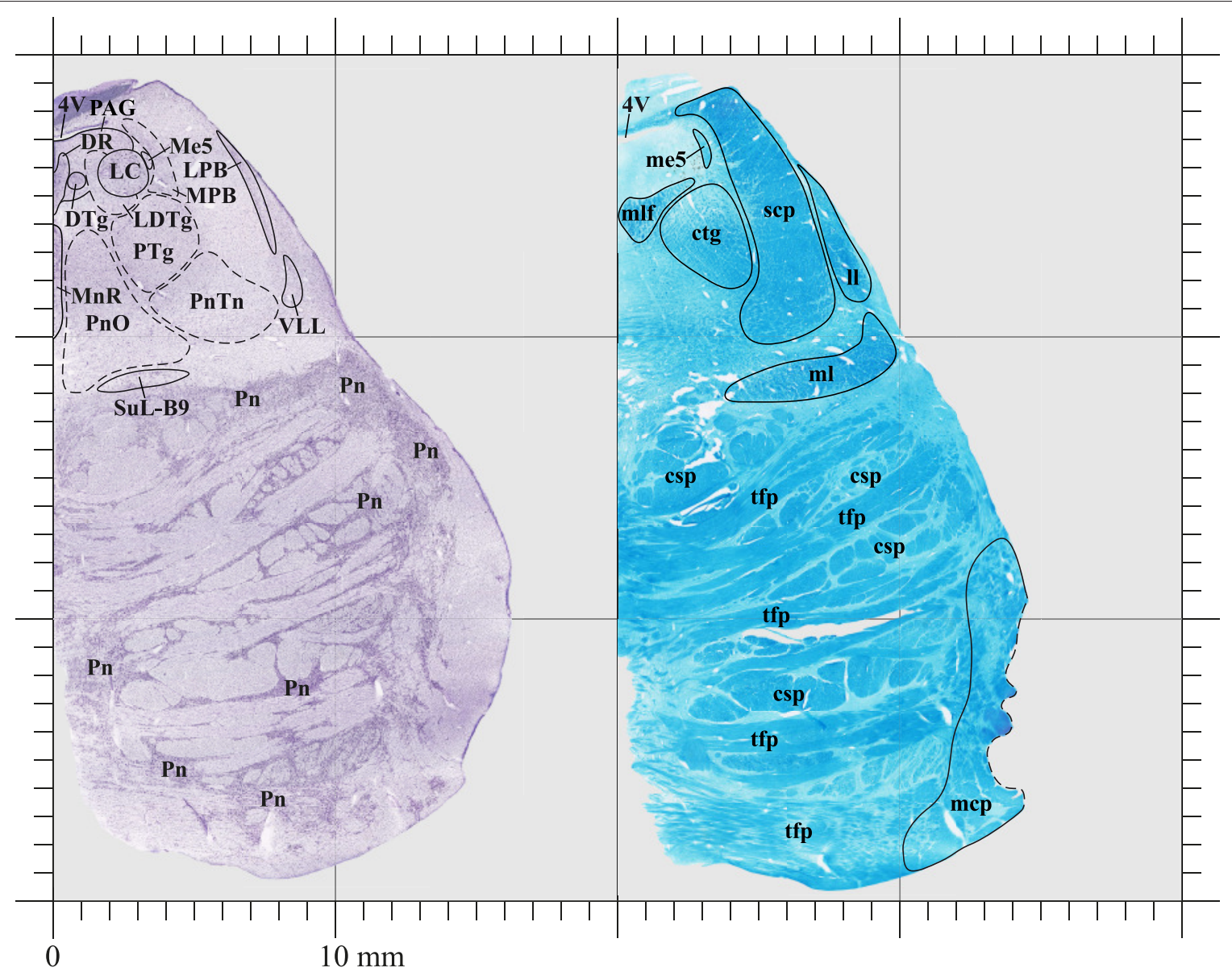

4V : $\quad$ Fourth ventricle

DR: Dorsal raphe nucleus

DTg : Dorsal tegmental nucleus

LC : Locus coeruleus

LDTg: Laterodorsal tegmental nucleus

LPB : Lateral parabrachial nucleus

Me5 : Mesencephalic trigeminal nucleus

MnR : Median raphe nucleus

MPB : Medial parabrachial nucleus

PAG : Periaqueductal gray

Pn : $\quad$ Pontine nuclei

$\mathrm{PnO}$ : Pontine reticular nucleus, oral part

PnTn : Pontine reticular nucleus, tegmental part

PTg: Pedunculotegmental nucleus

SuL-B9 : Supralemniscal nucleus - B9 serotonin cells

VLL: Ventral nucleus of the lateral lemniscus

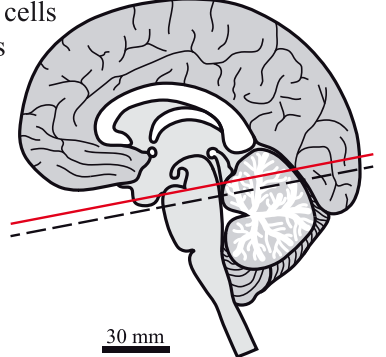

Plate 13
4V: $\quad$ Fourth ventricle

csp : Corticospinal tract

ctg : Central tegmental tract

11: Lateral lemniscus

mcp : Middle cerebellar peduncle

me5 : Mesencephalic trigeminal tract

$\mathrm{ml}$ : Medial lemniscus

mlf: Medial longitudinal fasciculus

scp : $\quad$ Superior cerebellar peduncle

tfp : Transverse fibers of the pons 


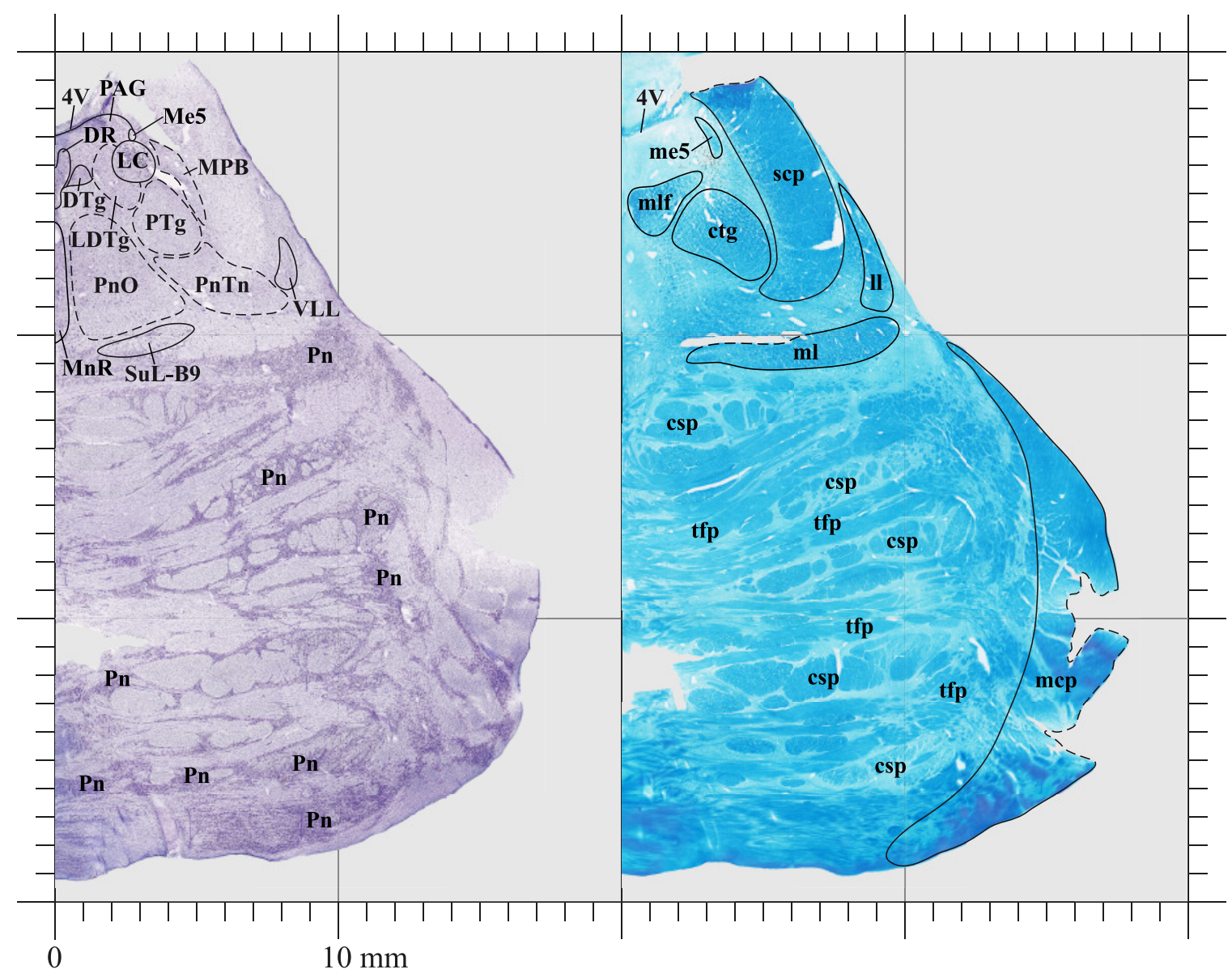

4V : $\quad$ Fourth ventricle

DR: Dorsal raphe nucleus

DTg : Dorsal tegmental nucleus

LC : Locus coeruleus

LDTg : Laterodorsal tegmental nucleus

Me5: Mesencephalic trigeminal nucleus

MnR: Median raphe nucleus

MPB : Medial parabrachial nucleus

PAG : Periaqueductal gray

Pn : $\quad$ Pontine nuclei

$\mathrm{PnO}$ : Pontine reticular nucleus, oral part

PnTn : Pontine reticular nucleus, tegmental part

PTg: Pedunculotegmental nucleus

SuL-B9 : Supralemniscal nucleus - B9 serotonin cells

VLL: Ventral nucleus of the lateral lemniscus

Plate 14

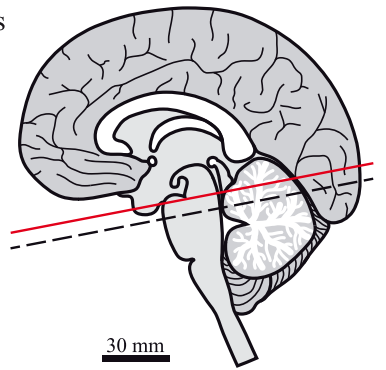

4V: $\quad$ Fourth ventricle

csp : Corticospinal tract

ctg : Central tegmental tract

11 : Lateral lemniscus

mcp : Middle cerebellar peduncle

me5 : Mesencephalic trigeminal tract

$\mathrm{ml}$ : $\quad$ Medial lemniscus

mlf: Medial longitudinal fasciculus

scp : $\quad$ Superior cerebellar peduncle

tfp : $\quad$ Transverse fibers of the pons 


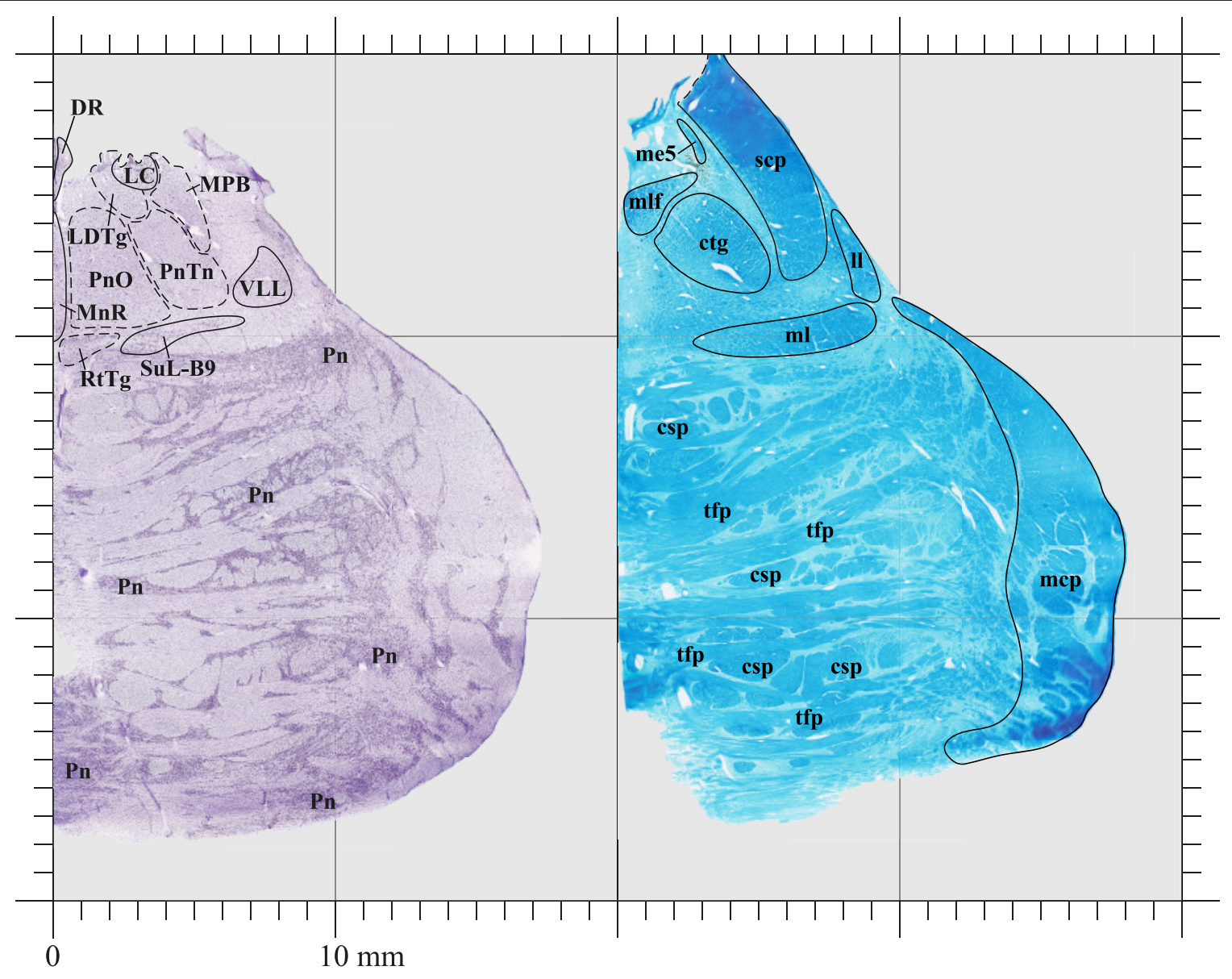

DR : Dorsal raphe nucleus

csp : Corticospinal tract

LC : Locus coeruleus

ctg : Central tegmental tract

LDTg : Laterodorsal tegmental nucleus

MnR: Median raphe nucleus

11: Lateral lemniscus

MPB : Medial parabrachial nucleus

Pn : $\quad$ Pontine nuclei

mcp : Middle cerebellar peduncle

me5: Mesencephalic trigeminal tract

PnO : Pontine reticular nucleus, oral part

PnTn : Pontine reticular nucleus, tegmental part

RtTg : Reticulotegmental nucleus

SuL-B9 : Supralemniscal nucleus - B9 serotonin cells

$\mathrm{ml}$ : Medial lemniscus

mlf: $\quad$ Medial longitudinal fasciculus

scp : $\quad$ Superior cerebellar peduncle

VLL: Ventral nucleus of the lateral lemniscus

tfp : Transverse fibers of the pons

\section{Plate 15}

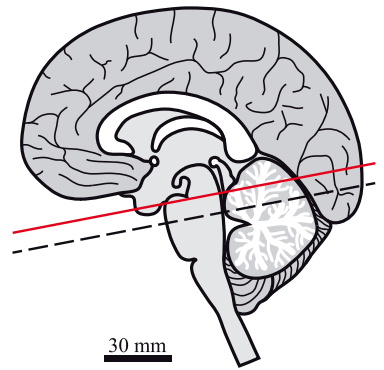

$-10.64 \mathrm{~mm}$ 


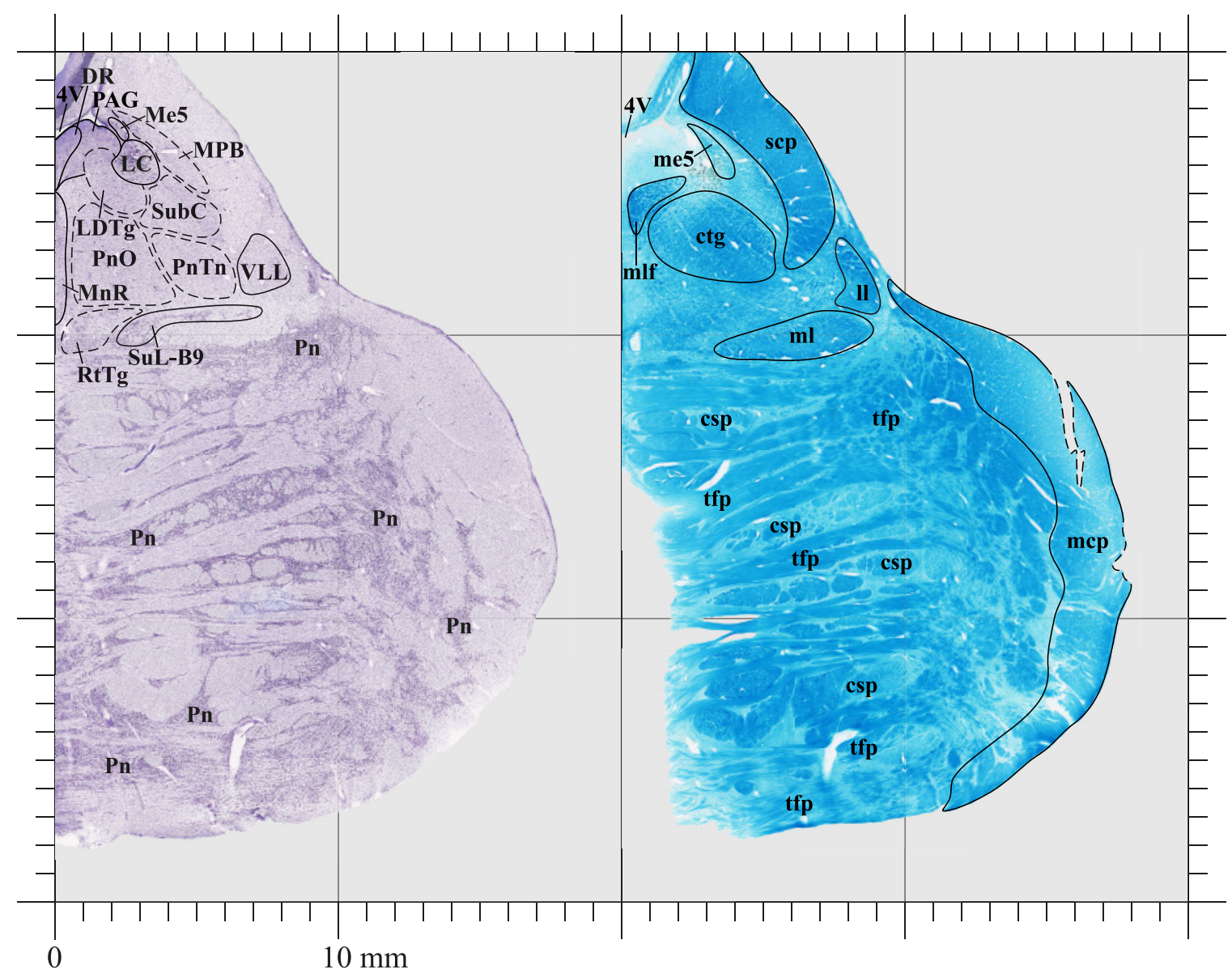

4V : $\quad$ Fourth ventricle

DR : Dorsal raphe nucleus

4V: $\quad$ Fourth ventricle

LC : Locus coeruleus

LDTg: Laterodorsal tegmental nucleus

Me5: Mesencephalic trigeminal nucleus

MnR: Median raphe nucleus

MPB : Medial parabrachial nucleus

PAG : Periaqueductal gray

Pn : Pontine nuclei

$\mathrm{PnO}$ : Pontine reticular nucleus, oral part

csp : Corticospinal tract

ctg : Central tegmental tract

11 : Lateral lemniscus

mcp : Middle cerebellar peduncle

me5 : Mesencephalic trigeminal tract

$\mathrm{ml}$ : Medial lemniscus

mlf : Medial longitudinal fasciculus

PnTn : Pontine reticular nucleus, tegmental part

scp : $\quad$ Superior cerebellar peduncle

RtTg: Reticulotegmental nucleus

SubC : Subcoeruleus nucleus

SuL-B9 : Supralemniscal nucleus - B9 serotonin cells

VLL: Ventral nucleus of the lateral lemniscus

Plate 16

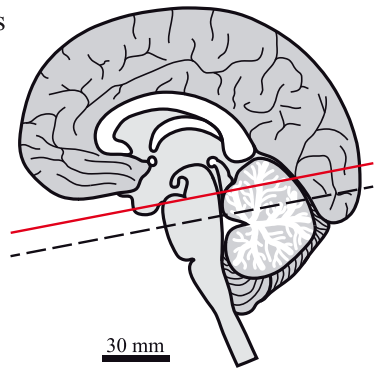

$-13.05 \mathrm{~mm}$ 


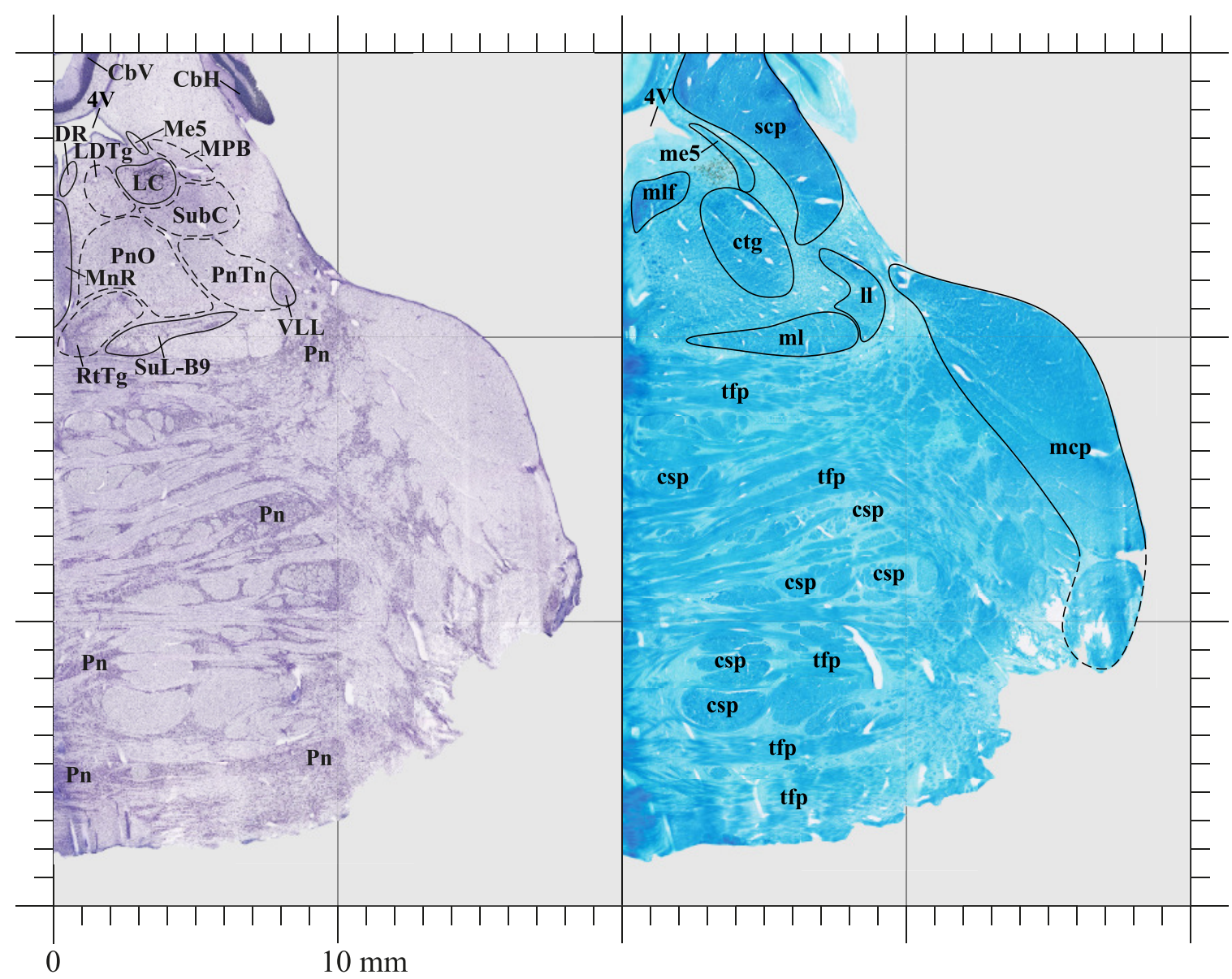

4V: $\quad$ Fourth ventricle

$\mathrm{CbH}$ : Cerebellar hemisphere

$\mathrm{CbV}$ : Cerebellar vermis

DR : Dorsal raphe nucleus

LC : Locus coeruleus

LDTg : Laterodorsal tegmental nucleus

Me5: Mesencephalic trigeminal nucleus

MnR: Median raphe nucleus

MPB : Medial parabrachial nucleus

Pn : $\quad$ Pontine nuclei

$\mathrm{PnO}$ : Pontine reticular nucleus, oral part

PnTn : Pontine reticular nucleus, tegmental part

RtTg: Reticulotegmental nucleus

SubC: Subcoeruleus nucleus

SuL-B9 : Supralemniscal nucleus - B9 serotonin cells

VLL: Ventral nucleus of the lateral lemniscus

Plate 17

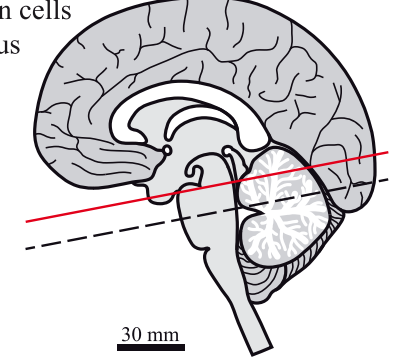

4V: $\quad$ Fourth ventricle

csp : Corticospinal tract

ctg : Central tegmental tract

11: Lateral lemniscus

mcp : Middle cerebellar peduncle

me5 : Mesencephalic trigeminal tract

$\mathrm{ml}$ : $\quad$ Medial lemniscus

mlf: Medial longitudinal fasciculus

scp : $\quad$ Superior cerebellar peduncle

tfp : $\quad$ Transverse fibers of the pons 


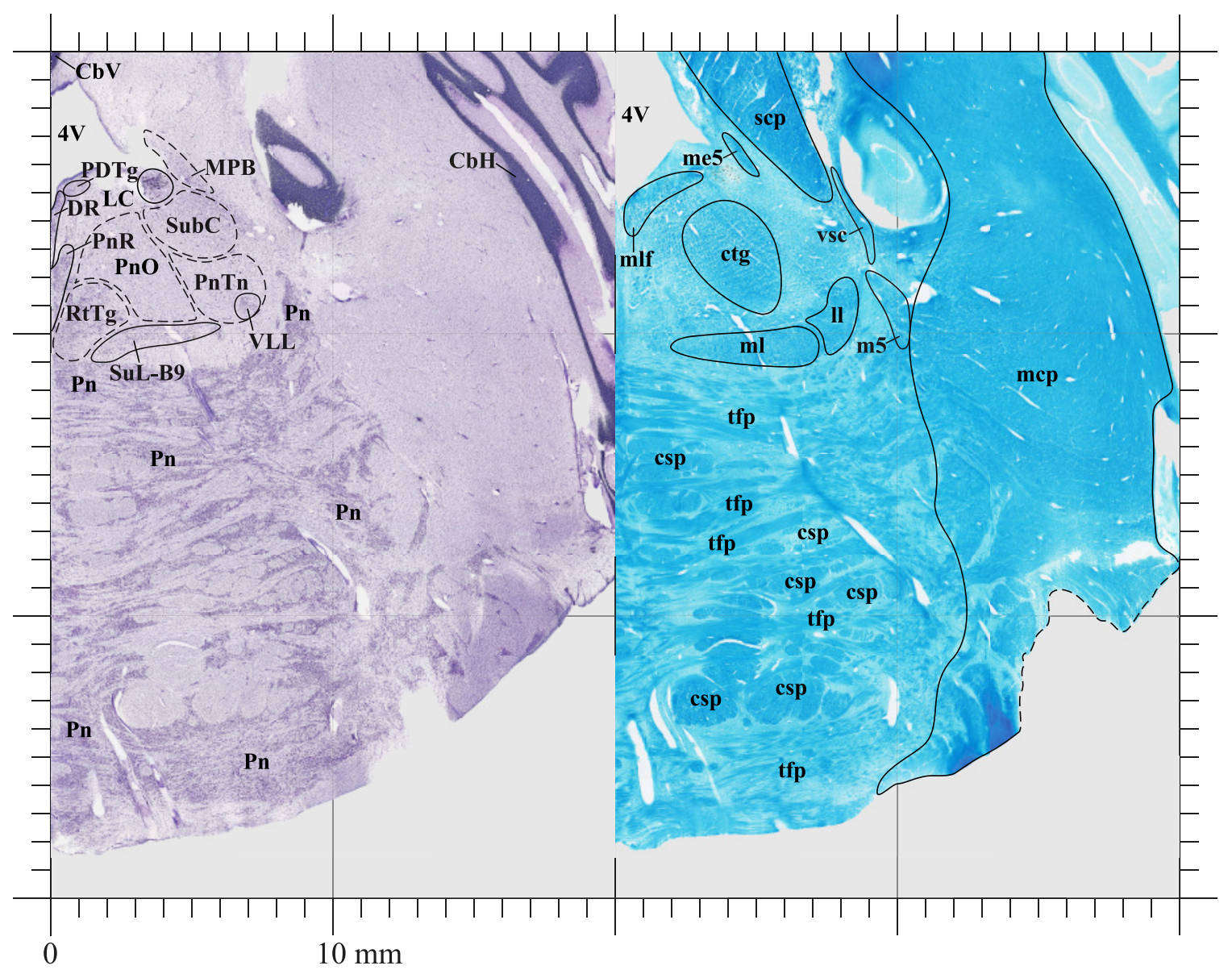

4V: $\quad$ Fourth ventricle

$\mathrm{CbH}$ : Cerebellar hemisphere

$\mathrm{CbV}$ : Cerebellar vermis

DR : Dorsal raphe nucleus

LC : Locus coeruleus

MPB : Medial parabrachial nucleus

PDTg : Posterodorsal tegmental nucleus

Pn : $\quad$ Pontine nuclei

$\mathrm{PnO}$ : Pontine reticular nucleus, oral part

PnR : Pontine raphe nucleus

PnTn : Pontine reticular nucleus, tegmental part

RtTg: Reticulotegmental nucleus

SubC: Subcoeruleus nucleus

SuL-B9 : Supralemniscal nucleus - B9 serotonin cells

VLL: Ventral nucleus of the lateral lemniscus

Plate 18

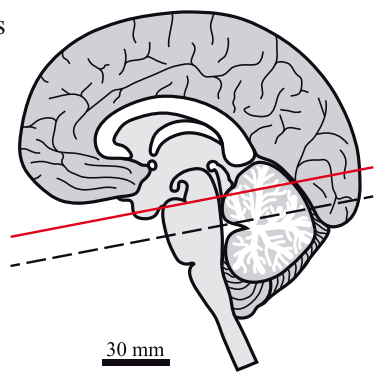

4V: $\quad$ Fourth ventricle

csp : Corticospinal tract

ctg : Central tegmental tract

11 : $\quad$ Lateral lemniscus

mcp : Middle cerebellar peduncle

m5: Motor root of the trigeminal nerve

me5: Mesencephalic trigeminal tract

$\mathrm{ml}$ : $\quad$ Medial lemniscus

mlf : Medial longitudinal fasciculus

scp : $\quad$ Superior cerebellar peduncle

tfp : $\quad$ Transverse fibers of the pons

vsc : Ventral spinocerebellar tract 


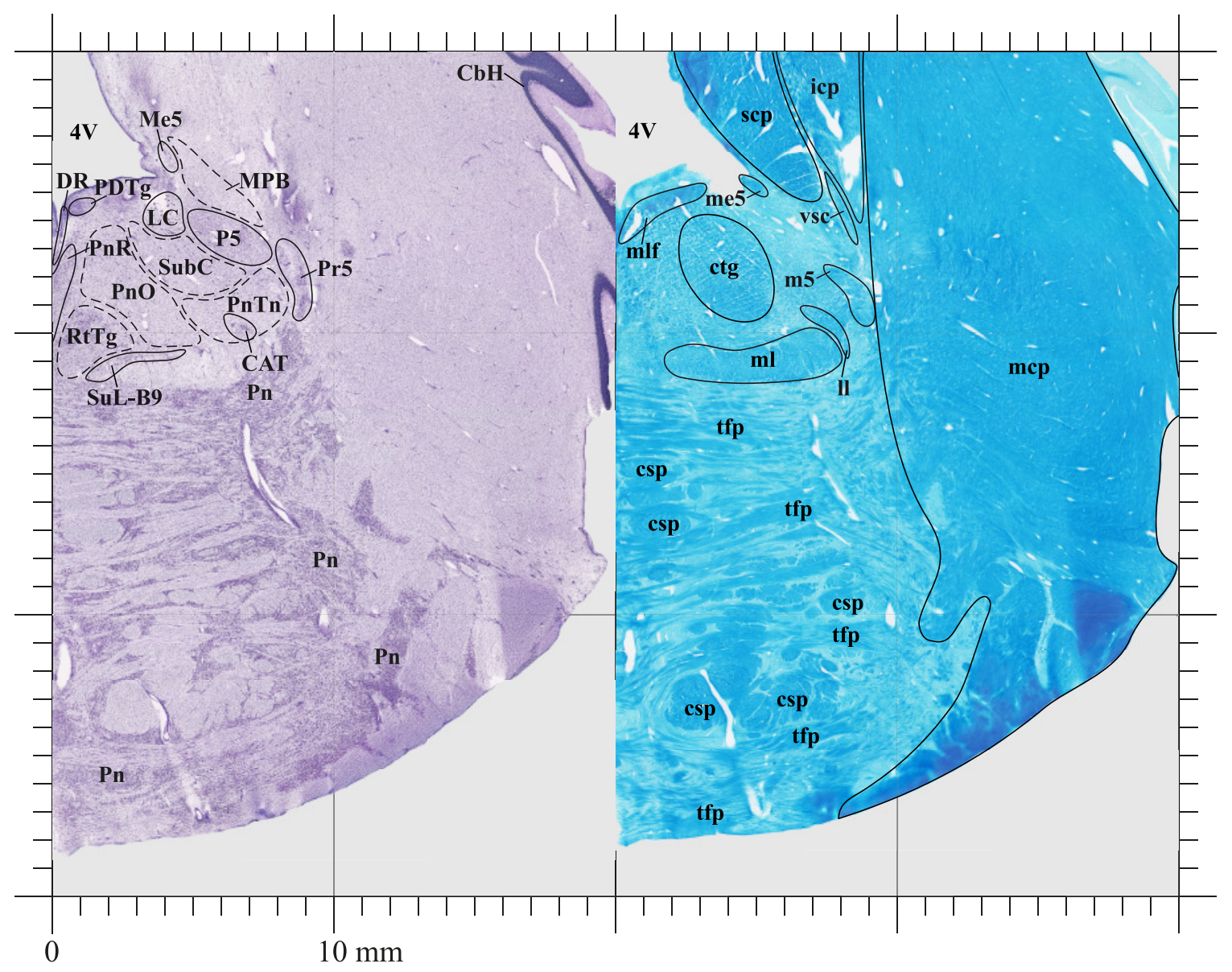

4V : Fourth ventricle

CAT : Nucleus of the central acoustic tract

$\mathrm{CbH}$ : Cerebellar hemisphere

DR : Dorsal raphe nucleus

LC : Locus coeruleus

Me5: Mesencephalic trigeminal nucleus

MPB : Medial parabrachial nucleus

P5 : $\quad$ Peritrigeminal zone

PDTg: Posterodorsal tegmental nucleus

Pn : Pontine nuclei

PnO : Pontine reticular nucleus, oral part

PnR : Pontine raphe nucleus

PnTn : Pontine reticular nucleus, tegmental part

Pr5 : Principal sensory trigeminal nucleus

RtTg: Reticulotegmental nucleus

SubC : Subcoeruleus nucleus

SuL-B9 : Supralemniscal nucleus - B9 serotonin cells

Plate 19

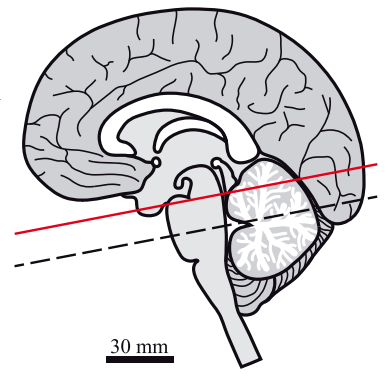

4V : $\quad$ Fourth ventricle

csp : Corticospinal tract

ctg : Central tegmental tract

icp : Inferior cerebellar peduncle

11: $\quad$ Lateral lemniscus

m5 : Motor root of the trigeminal nerve

mcp : Middle cerebellar peduncle

me5: Mesencephalic trigeminal tract

$\mathrm{ml}$ : Medial lemniscus

mlf: Medial longitudinal fasciculus

scp : $\quad$ Superior cerebellar peduncle

tfp : Transverse fibers of the pons

vsc : Ventral spinocerebellar tract 


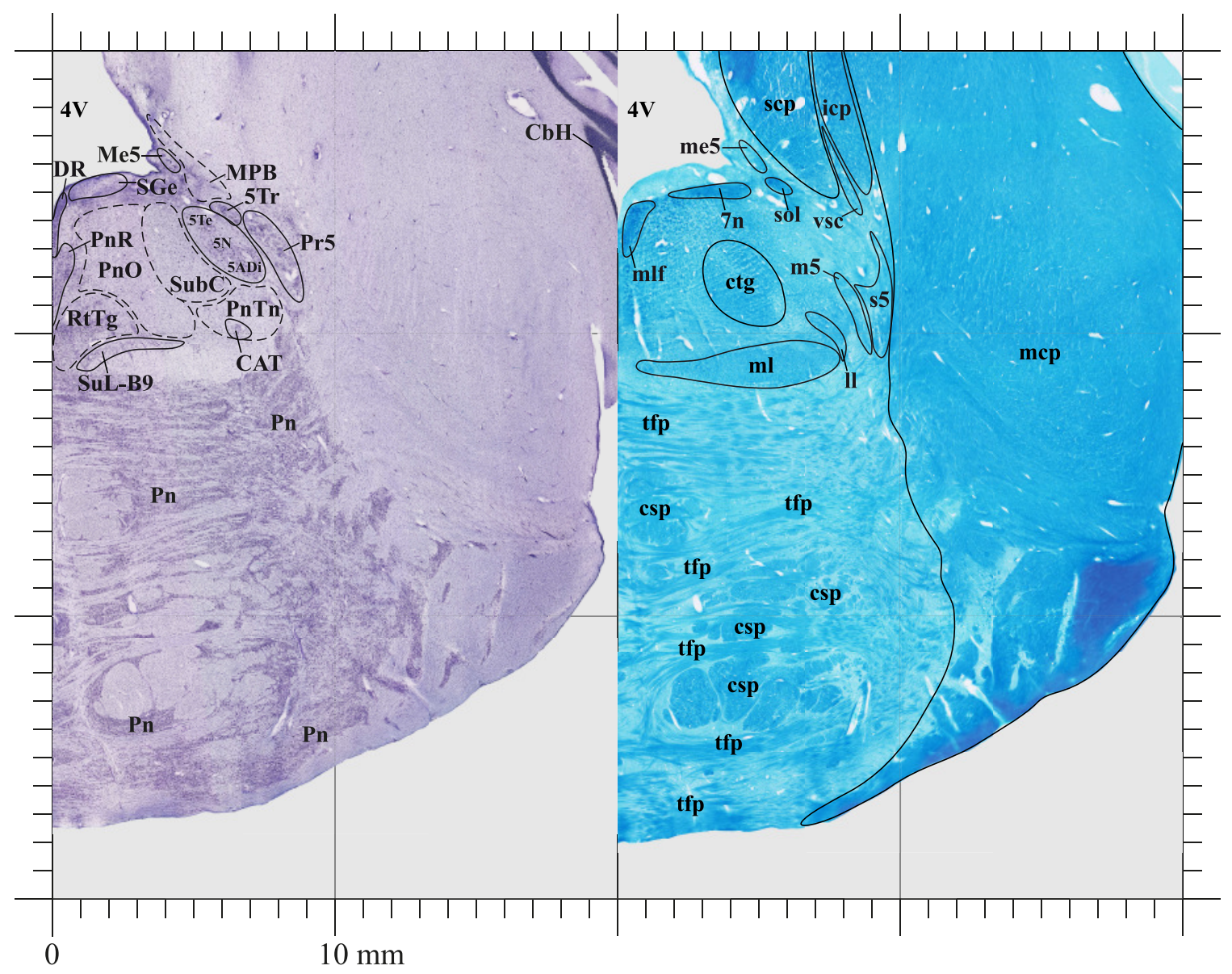

4V: $\quad$ Fourth ventricle

5ADi : Motor trigeminal nucleus, anterior digastric part

$5 \mathrm{~N}$ : $\quad$ Motor trigeminal nucleus

4V: $\quad$ Fourth ventricle

5Te : Motor trigeminal nucleus, temporal part

5Tr: Trigeminal transition zone

CAT : Nucleus of the central acoustic tract

$\mathrm{CbH}$ : Cerebellar hemisphere

DR : Dorsal raphe nucleus

Me5: Mesencephalic trigeminal nucleus

MPB : Medial parabrachial nucleus

Pn : $\quad$ Pontine nuclei

7n: $\quad$ Facial nerve

$\mathrm{PnO}$ : Pontine reticular nucleus, oral part

PnR : Pontine raphe nucleus

PnTn : Pontine reticular nucleus, tegmental part

Pr5 : Principal sensory trigeminal nucleus

RtTg: Reticulotegmental nucleus

SGe : Supragenual nucleus of the raphe

SubC: Subcoeruleus nucleus

SuL-B9 : Supralemniscal nucleus - B9 serotonin cells

Plate 20

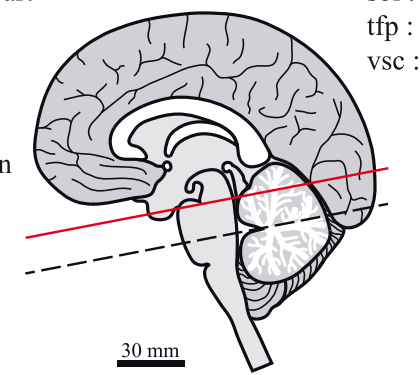

Corticospinal tract

ctg : Central tegmental tract

icp : Inferior cerebellar peduncle

11 : Lateral lemniscus

m5 : Motor root of the trigeminal nerve

mcp : Middle cerebellar peduncle

me5 : Mesencephalic trigeminal tract

$\mathrm{ml}$ : $\quad$ Medial lemniscus

mlf: Medial longitudinal fasciculus

s5: Sensory root of the trigeminal nerve

scp : Superior cerebellar peduncle

sol : $\quad$ Solitary tract

tfp : Transverse fibers of the pons

Ventral spinocerebellar tract 


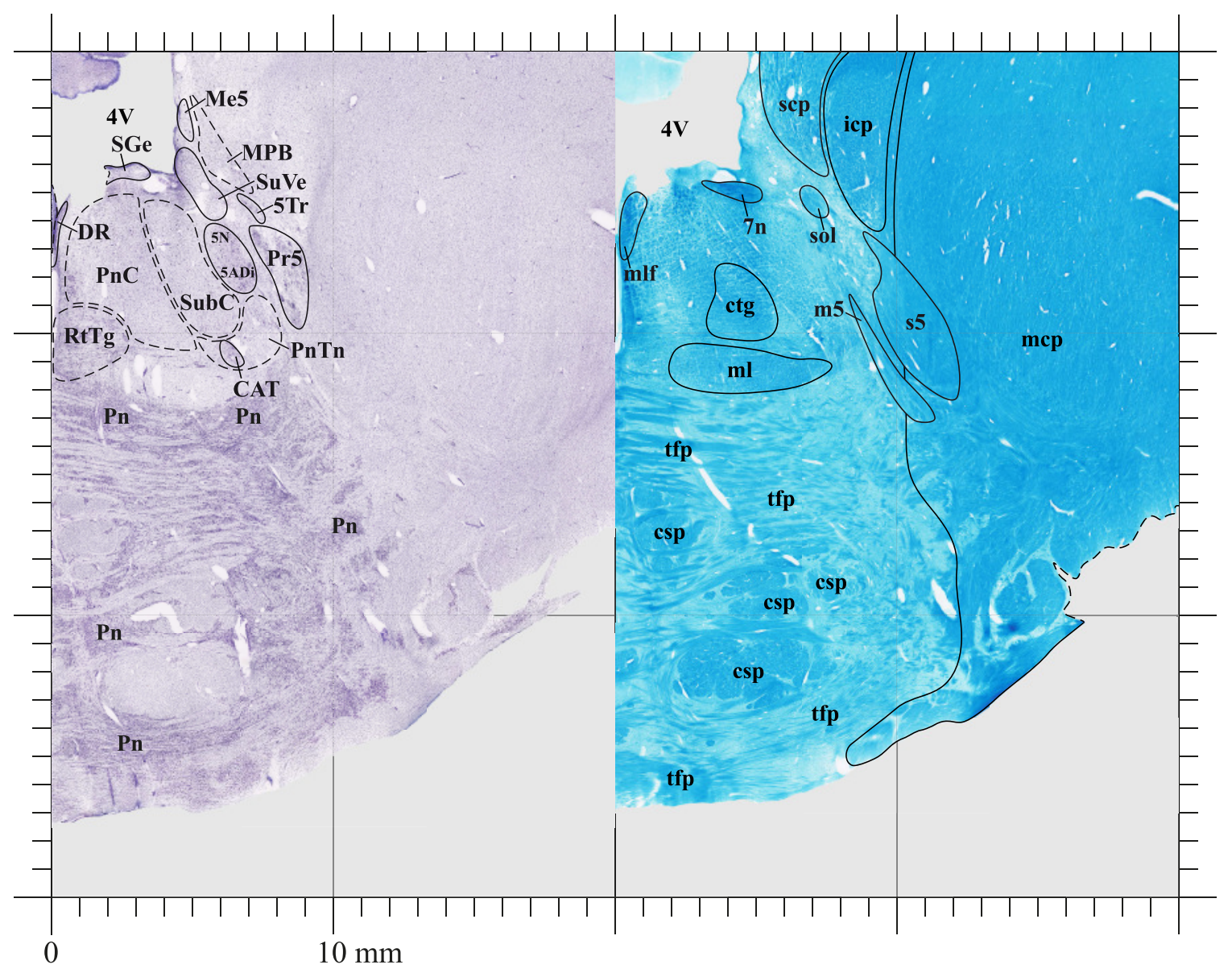

4V: $\quad$ Fourth ventricle

5ADi : Motor trigeminal nucleus, anterior digastric part

$5 \mathrm{~N}$ : $\quad$ Motor trigeminal nucleus

5Tr: Trigeminal transition zone

CAT : Nucleus of the central acoustic tract

DR : Dorsal raphe nucleus

Me5 : Mesencephalic trigeminal nucleus

MPB : Medial parabrachial nucleus

Pn : $\quad$ Pontine nuclei

PnC : Pontine reticular nucleus, caudal part

PnTn : Pontine reticular nucleus, tegmental part

Pr5 : Principal sensory trigeminal nucleus

RtTg: Reticulotegmental nucleus

SGe : Supragenual nucleus of the raphe

SubC: Subcoeruleus nucleus

SuVe: Superior vestibular nucleus
Plate 21
4V: $\quad$ Fourth ventricle

7n: $\quad$ Facial nerve

csp : Corticospinal tract

ctg : Central tegmental tract

icp : Inferior cerebellar peduncle

m5 : Motor root of the trigeminal nerve

mcp : Middle cerebellar peduncle

$\mathrm{ml}$ : Medial lemniscus

mlf: Medial longitudinal fasciculus

s5: $\quad$ Sensory root of the trigeminal nerve

scp : $\quad$ Superior cerebellar peduncle

sol : Solitary tract

tfp : $\quad$ Transverse fibers of the pons 


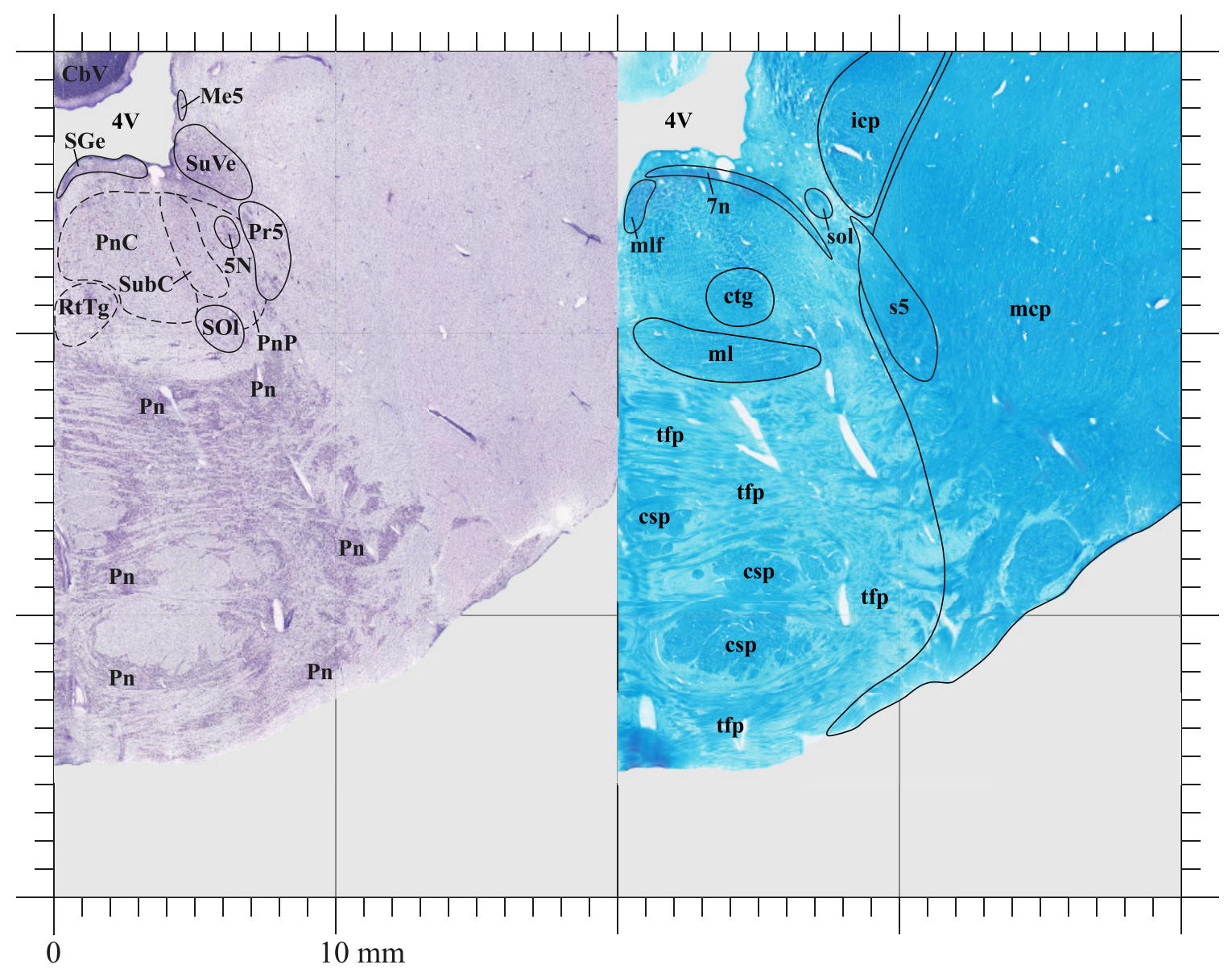

4V: $\quad$ Fourth ventricle

$5 \mathrm{~N}$ : $\quad$ Motor trigeminal nucleus

$\mathrm{CbV}$ : Cerebellar vermis

Me5: Mesencephalic trigeminal nucleus

Pn : $\quad$ Pontine nuclei

PnC : Pontine reticular nucleus, caudal part

PnP : Pontine reticular nucleus, parvocellular part

Pr5 : $\quad$ Principal sensory trigeminal nucleus

RtTg : Reticulotegmental nucleus

SGe : Supragenual nucleus

SOl : Superior olivary nucleus

SubC : Subcoeruleus nucleus

SuVe: Superior vestibular nucleus
4V : $\quad$ Fourth ventricle

7n: $\quad$ Facial nerve

csp : Corticospinal tract

ctg : Central tegmental tract

icp : Inferior cerebellar peduncle

mcp : Middle cerebellar peduncle

$\mathrm{ml}$ : $\quad$ Medial lemniscus

mlf: Medial longitudinal fasciculus

s5 : $\quad$ Sensory root of the trigeminal nerve

sol : $\quad$ Solitary tract

tfp : Transverse fibers of the pons

Plate 22

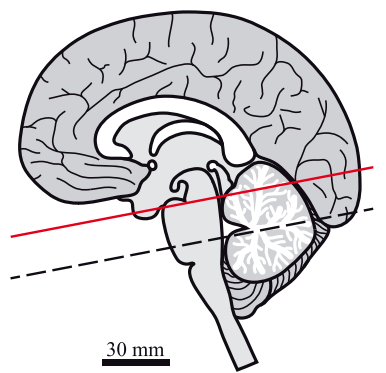

$-19.36 \mathrm{~mm}$ 


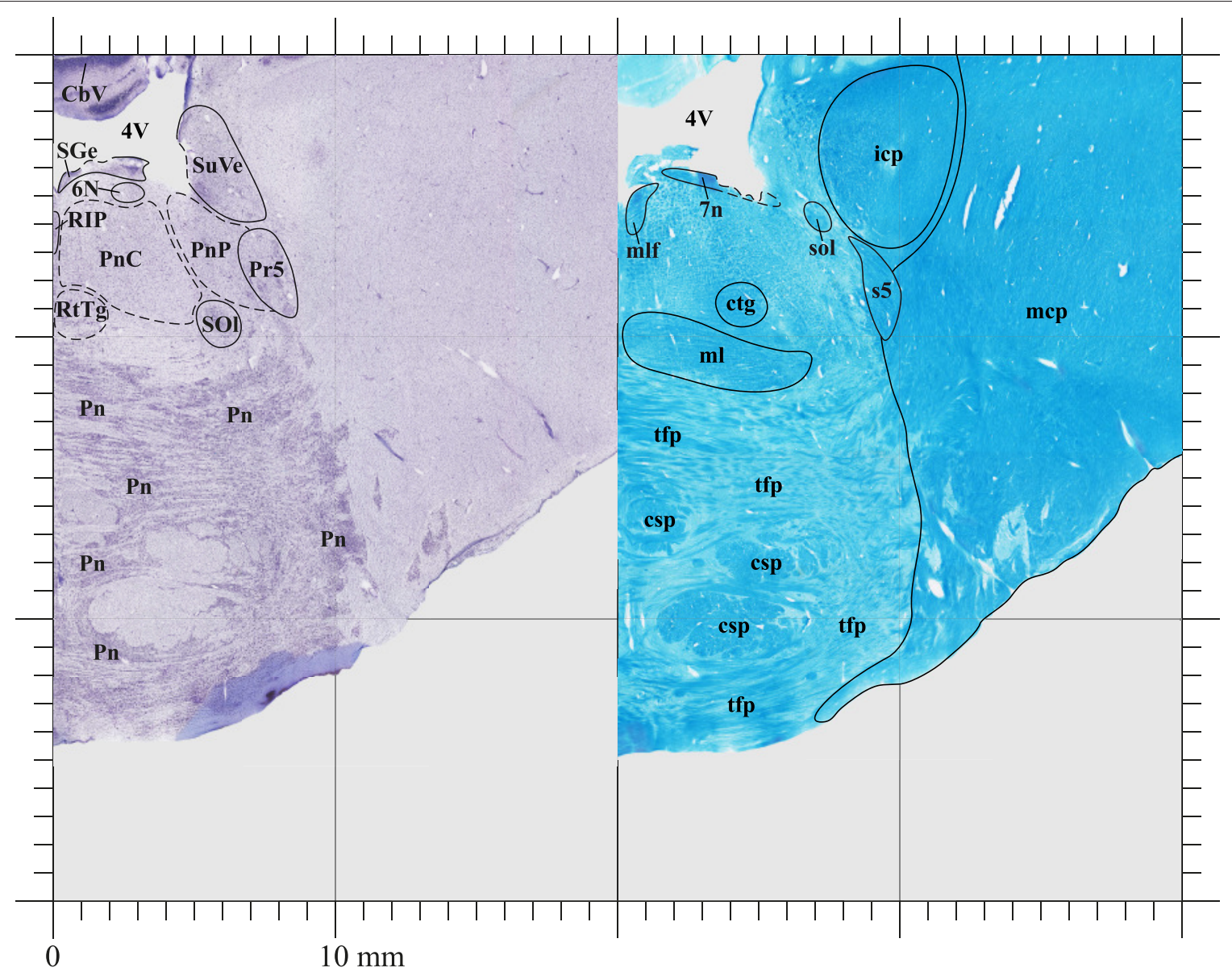

4V : $\quad$ Fourth ventricle

6N: Abducens nucleus

4V: $\quad$ Fourth ventricle

$\mathrm{CbV}$ : Cerebellar vermis

7n: $\quad$ Facial nerve

Pn : Pontine nuclei

PnC : Pontine reticular nucleus, caudal part

PnP : Pontine reticular nucleus, parvocellular part

Pr5 : Principal sensory trigeminal nucleus

RIP : Raphe interpositus nucleus

RtTg: Reticulotegmental nucleus

SGe : Supragenual nucleus

SO1 : Superior olivary nucleus

SuVe : Superior vestibular nucleus

csp : Corticospinal tract

ctg : Central tegmental tract

icp : Inferior cerebellar peduncle

mcp : Middle cerebellar peduncle

$\mathrm{ml}$ : Medial lemniscus

mlf: Medial longitudinal fasciculus

s5 : $\quad$ Sensory root of the trigeminal nerve

sol : $\quad$ Solitary tract

tfp : Transverse fibers of the pons

Plate 23

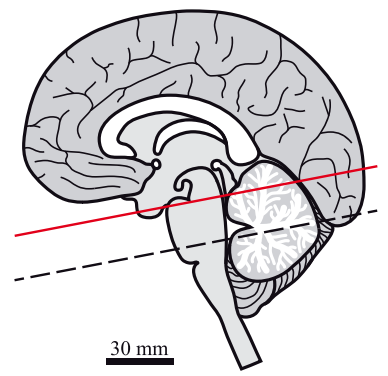

$-20.34 \mathrm{~mm}$ 


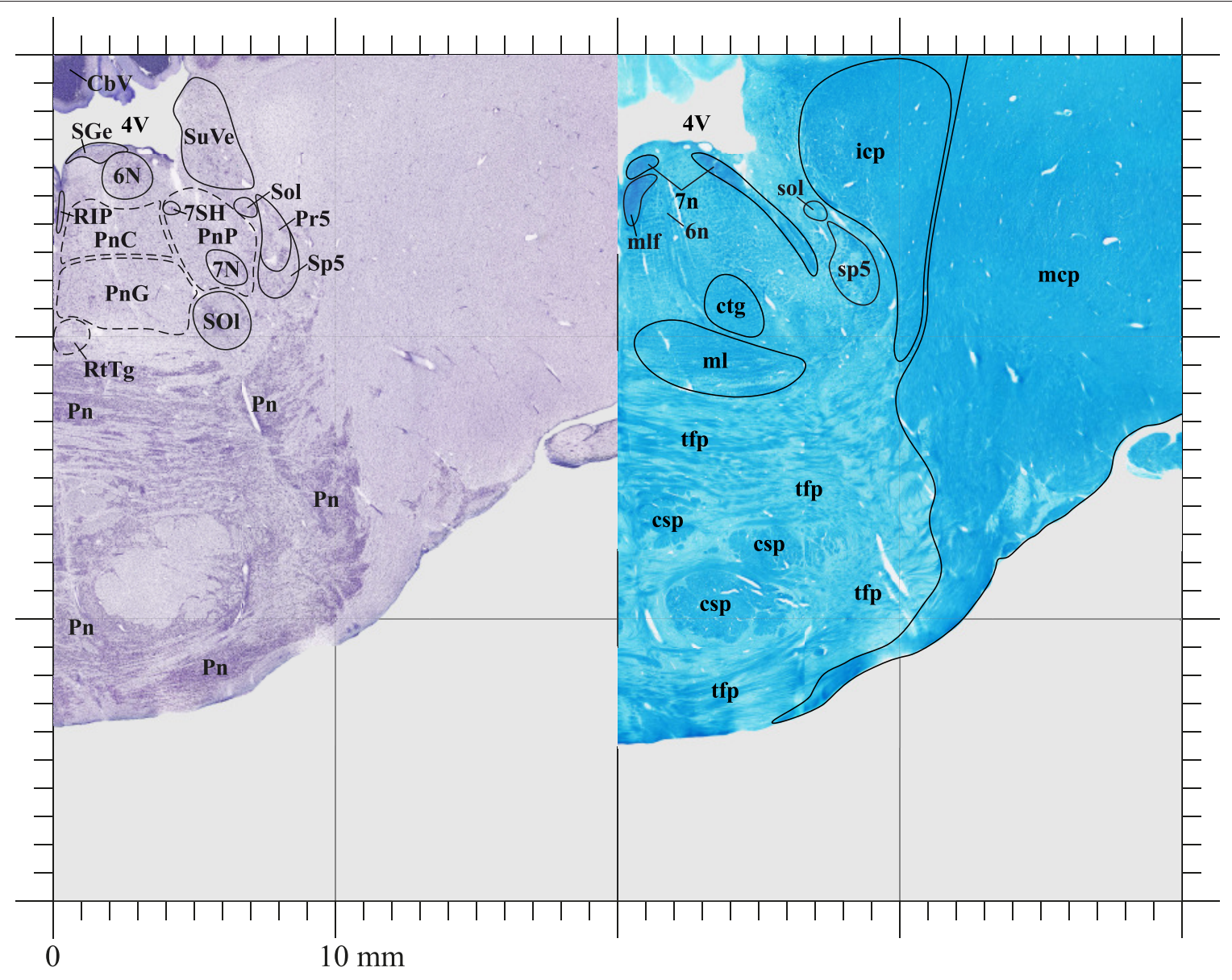

4V: $\quad$ Fourth ventricle

$6 \mathrm{~N}$ : $\quad$ Abducens nucleus

7N : Facial nucleus

7SH : $\quad$ Facial nucleus, stylohyoid part

CbV: Cerebellar vermis

Pn : Pontine nuclei

PnC : Pontine reticular nucleus, caudal part

PnG : Pontine reticular nucleus, gigantocellular part

PnP : Pontine reticular nucleus, parvocellular part

Pr5 : Principal sensory trigeminal nucleus

RIP : Raphe interpositus nucleus

RtTg: Reticulotegmental nucleus

4V: $\quad$ Fourth ventricle

6n: Abducens nerve

7n: $\quad$ Facial nerve

csp : Corticospinal tract

ctg : Central tegmental tract

icp : Inferior cerebellar peduncle

mcp : Middle cerebellar peduncle

$\mathrm{ml}$ : Medial lemniscus

mlf: Medial longitudinal fasciculus

sp5 : Spinal trigeminal tract

SGe : Supragenual nucleus

SOl : $\quad$ Superior olivary nucleus

Sol : $\quad$ Solitary nucleus

Sp5: Spinal trigeminal nucleus

SuVe : Superior vestibular nucleus

Plate 24

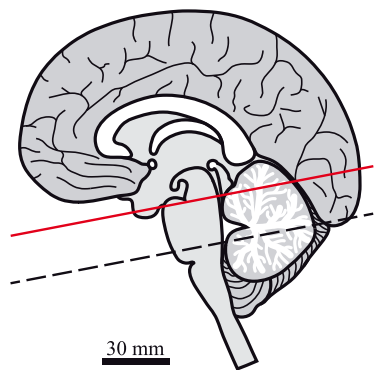




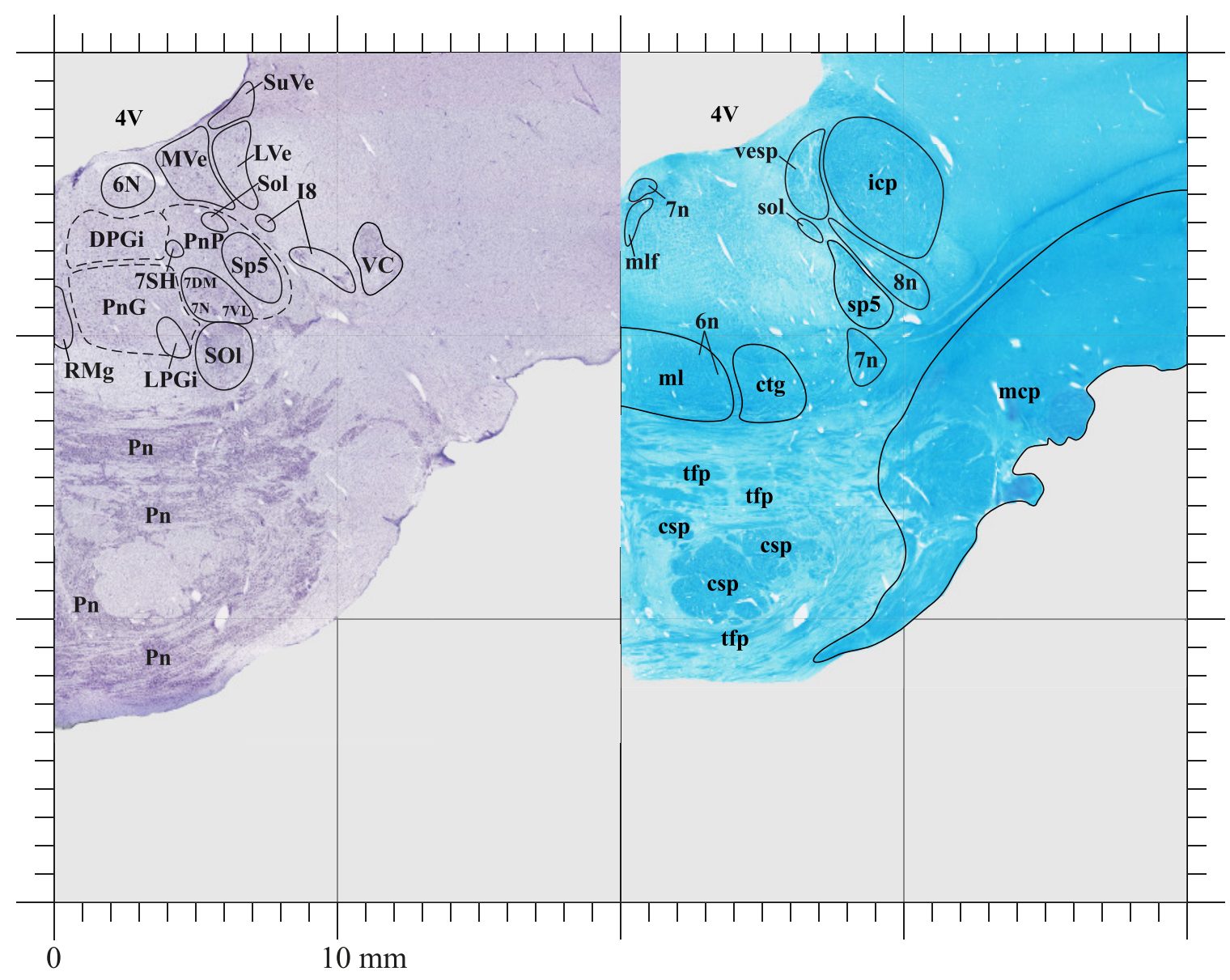

4V: $\quad$ Fourth ventricle

$6 \mathrm{~N}$ : $\quad$ Abducens nucleus

7DM : Facial nucleus, dorsomedial part

7N: $\quad$ Facial nucleus

7SH : Facial nucleus, stylohyoid part

7VL: Facial nucleus, ventrolateral part

DPGi : Dorsal paragigantocelular nucleus

I8: Interstitial nucleus of the vestibular part of the 8th nerve

LPGi : Lateral paragigantocellular nucleus

LVe: Lateral vestibular nucleus

MVe : Medial vestibular nucleus

Pn : $\quad$ Pontine nuclei

PnG : Pontine reticular nucleus, gigantocellular part

PnP : Pontine reticular nucleus, parvocellular part

$\mathrm{RMg}$ : Raphe magnus nucleus

SOl : $\quad$ Superior olivary nucleus

Sol : $\quad$ Solitary nucleus

Sp5: Spinal trigeminal nucleus

SuVe: Superior vestibular nucleus

VC: Ventral cochlear nucleus
4V: $\quad$ Fourth ventricle

6n: Abducens nerve

7n: $\quad$ Facial nerve

8n: Vestibulocochlear nerve

csp : Corticospinal tract

ctg : Central tegmental tract

icp : Inferior cerebellar peduncle

mcp : Middle cerebellar peduncle

$\mathrm{ml}$ : $\quad$ Medial lemniscus

mlf: $\quad$ Medial longitudinal fasciculus

sol : Solitary tract

sp5: Spinal trigeminal tract

tfp : Transverse fibers of the pons

vesp : Vestibulospinal tract

\section{Plate 25}




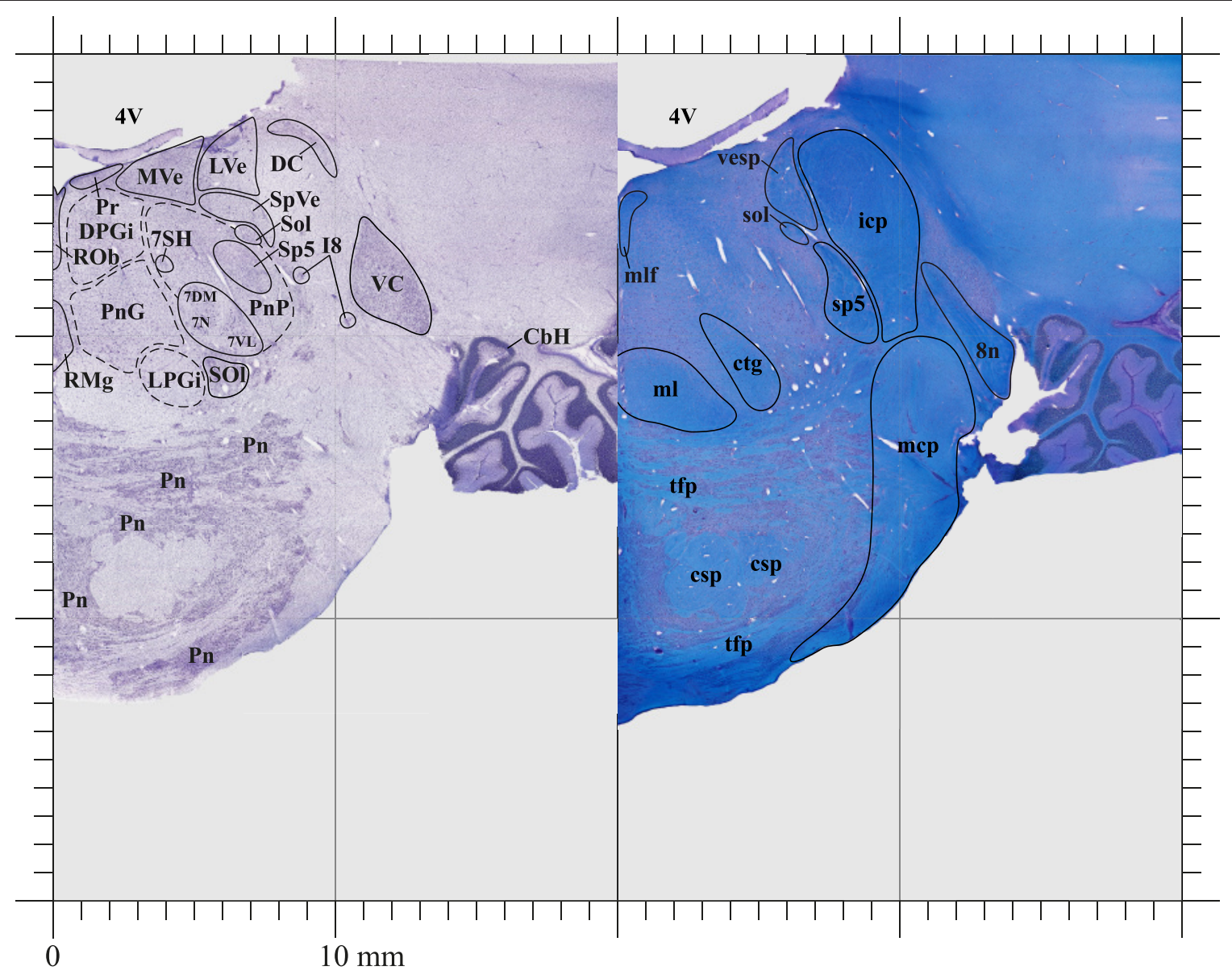

4V: $\quad$ Fourth ventricle

7DM : Facial nucleus, dorsomedial part

7N : $\quad$ Facial nucleus

7SH : Facial nucleus, stylohyoid part

7VL: Facial nucleus, ventrolateral part

$\mathrm{CbH}$ : Cerebellar hemisphere

DC : Dorsal cochlear nucleus

DPGi : Dorsal paragigantocelular nucleus

I8: Interstitial nucleus of the vestibular part of the 8th nerve

LPGi : Lateral paragigantocellular nucleus

LVe: Lateral vestibular nucleus

MVe: Medial vestibular nucleus

4V: $\quad$ Fourth ventricle

8n: Vestibulocochlear nerve

csp : Corticospinal tract

ctg : Central tegmental tract

icp : Inferior cerebellar peduncle

mcp : Middle cerebellar peduncle

$\mathrm{ml}$ : Medial lemniscus

mlf: Medial longitudinal fasciculus

sol : Solitary tract

sp5 : $\quad$ Spinal trigeminal tract

tfp : Transverse fibers of the pons

Pn : $\quad$ Pontine nuclei

vesp : Vestibulospinal tract

PnG : Pontine reticular nucleus, gigantocellular part

PnP : $\quad$ Pontine reticular nucleus, parvocellular part

Pr: Prepositus nucleus

$\mathrm{RMg}$ : Raphe magnus nucleus

ROb : Raphe obscurus nucleus

SOl : Superior olive

Sol : $\quad$ Solitary nucleus

Sp5: Spinal trigeminal nucleus

SpVe : Spinal vestibular nucleus

VC : Ventral cochlear nucleus

Plate 26

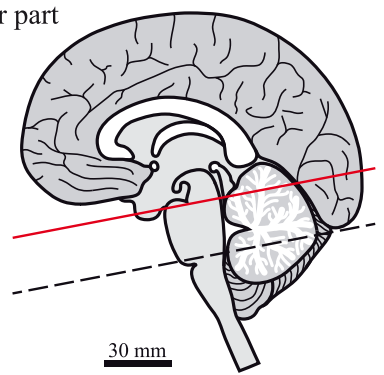

$-24.98 \mathrm{~mm}$ 


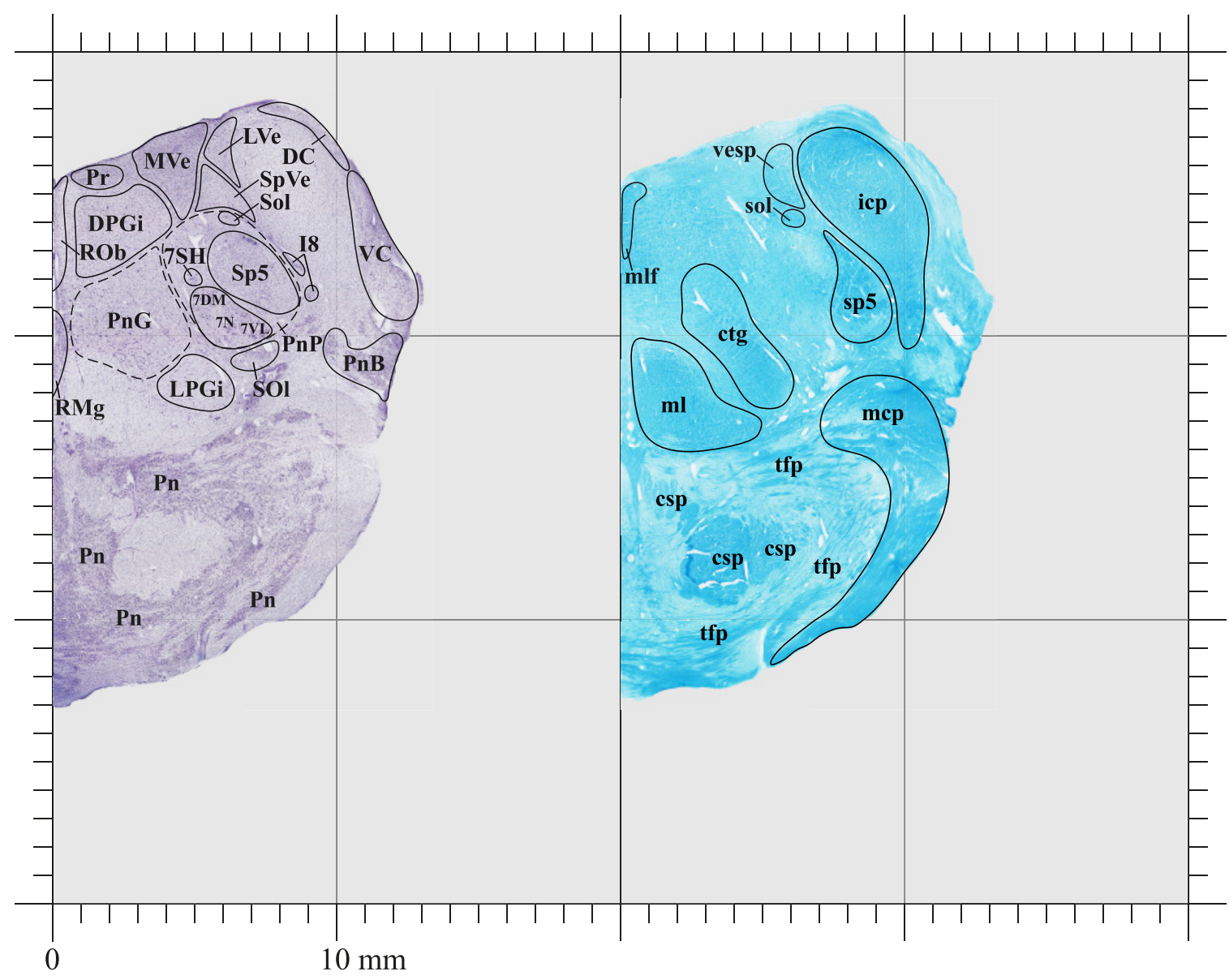

7DM : Facial nucleus, dorsomedial part

7N: $\quad$ Facial nucleus

7SH : $\quad$ Facial nucleus, stylohyoid part

7VL: Facial nucleus, ventrolateral part

DC : Dorsal cochlear nucleus

DPGi : Dorsal paragigantocellular nucleus

I8: Interstitial nucleus of the vestibular part of the 8th nerve

LPGi : Lateral paragigantocellular nucleus

LVe: Lateral vestibular nucleus

MVe: Medial vestibular nucleus

csp : Corticospinal tract

ctg : Central tegmental tract

icp : Inferior cerebellar peduncle

mcp : Middle cerebellar peduncle

$\mathrm{ml}$ : $\quad$ Medial lemniscus

mlf : Medial longitudinal fasciculus

sol : $\quad$ Solitary tract

sp5: Spinal trigeminal tract

Pn : Pontine nuclei

PnB : Pontobulbar nucleus

PnG : Pontine reticular nucleus, gigantocellular part

PnP : Pontine reticular nucleus, parvocellular part

Pr: Prepositus nucleus

RMg : Raphe magnus nucleus

ROb : Raphe obscurus nucleus

SOl : Superior olive

Sol : $\quad$ Solitary nucleus

Sp5 : Spinal trigeminal nucleus

SpVe: Spinal vestibular nucleus

VC: Ventral cochlear nucleus

\section{Plate 27}

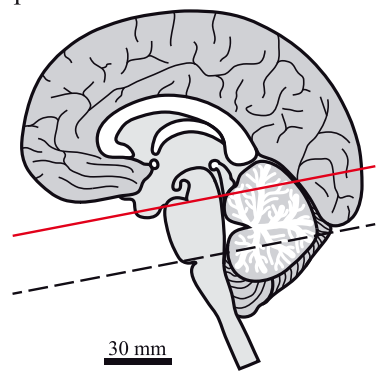

$-25.86 \mathrm{~mm}$ 


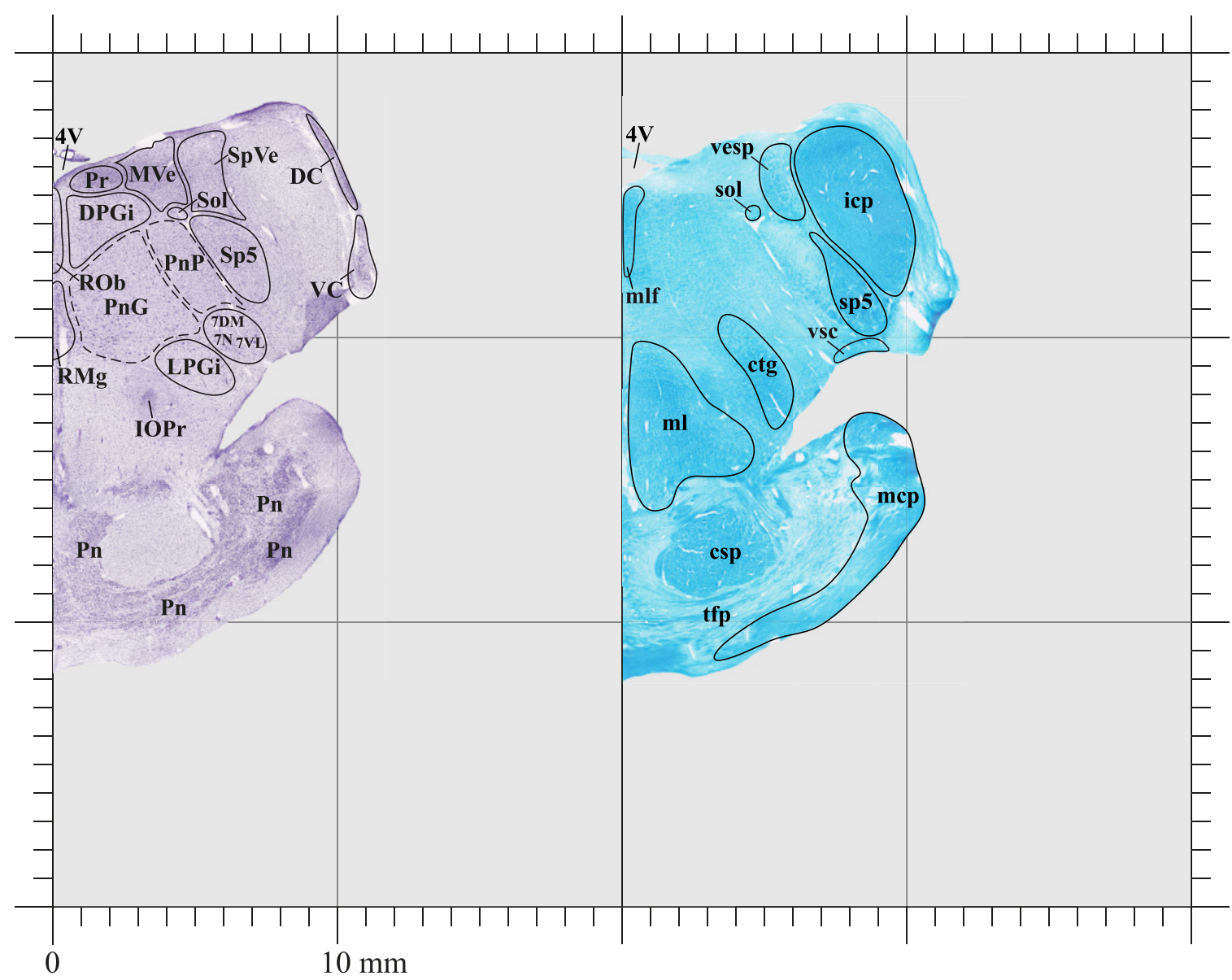

4V: $\quad$ Fourth ventricle

7DM : Facial nucleus, dorsomedial part

7N : $\quad$ Facial nucleus

4V : Fourth ventricle

7VL: Facial nucleus, ventrolateral part

csp : Corticospinal tract

DC : Dorsal cochlear nucleus

ctg : Central tegmental tract

DPGi : Dorsal paragigantocellular nucleus

icp : Inferior cerebellar peduncle

mcp : Middle cerebellar peduncle

IOPr : Inferior olive, principal nucleus

$\mathrm{ml}$ : $\quad$ Medial lemniscus

LPGi : Lateral paragigantocellular nucleus

mlf: Medial longitudinal fasciculus

sol : $\quad$ Solitary tract

MVe: Medial vestibular nucleus

sp5 : $\quad$ Spinal trigeminal tract

$\begin{array}{ll}\mathrm{Pn}: & \text { Pontine nuclei } \\ \mathrm{PnG}: & \text { Pontine reticular nucleus, gigantocellular part }\end{array}$

tfp : Transverse fibers of the pons

$\begin{array}{ll}\text { PnG : } & \text { Pontine reticular nucleus, gigantocellular part } \\ \text { PnP : } & \text { Pontine reticular nucleus, parvocellular part }\end{array}$

vesp : Vestibulospinal tract

Pr: Prepositus nucleus

vsc: Ventral spinocerebellar tract

$\mathrm{RMg}$ : Raphe magnus nucleus

ROb : Raphe obscurus nucleus

Sol : $\quad$ Solitary nucleus

Sp5 : $\quad$ Spinal trigeminal nucleus

SpVe: Spinal vestibular nucleus

VC: Ventral cochlear nucleus

Plate 28

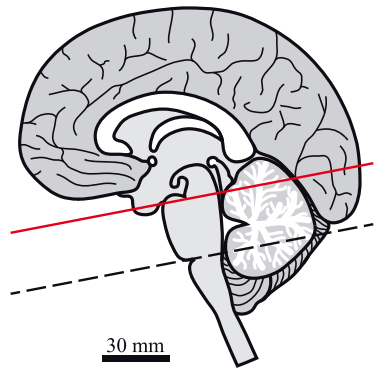




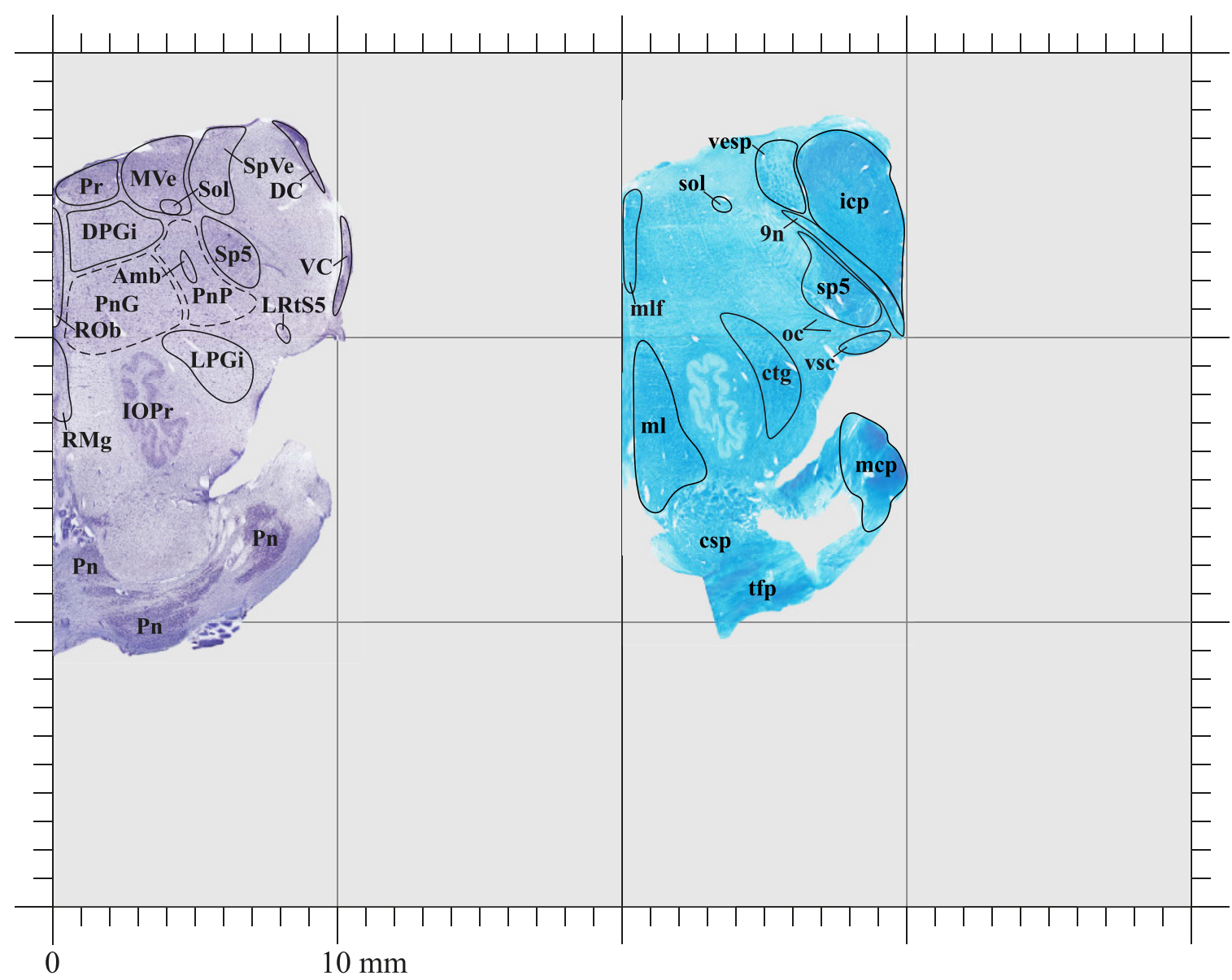

Amb : Ambiguus nucleus

DC : Dorsal cochlear nucleus

9n: Glossopharyngeal nerve

DPGi : Dorsal paragigantocellular nucleus

csp : Corticospinal tract

IOPr : Inferior olive, principal nucleus

LPGi : Lateral paragigantocellular nucleus

ctg : Central tegmental tract

LRtS5 : Lateral reticular nucleus, subtrigeminal part

icp : Inferior cerebellar peduncle

mcp : Middle cerebellar peduncle

MVe : Medial vestibular nucleus

Pn : Pontine nuclei

$\mathrm{ml}$ : $\quad$ Medial lemniscus

PnG : Pontine reticular nucleus, gigantocellular part

mlf: Medial longitudinal fasciculus

oc : Olivocerebellar tract

PnP : Pontine reticular nucleus, parvocellular part

sol : Solitary tract

Pr: $\quad$ Prepositus nucleus

sp5 : $\quad$ Spinal trigeminal tract

tfp : $\quad$ Transverse fibers of the pons

$\mathrm{RMg}$ : Raphe magnus nucleus

$\mathrm{ROb}$ : Raphe obscurus nucleus

vesp : Vestibulospinal tract

Sol : $\quad$ Solitary nucleus

vsc : Ventral spinocerebellar tract

Sp5: $\quad$ Spinal trigeminal nucleus

SpVe: Spinal vestibular nucleus

VC: Ventral cochlear nucleus

Plate 29

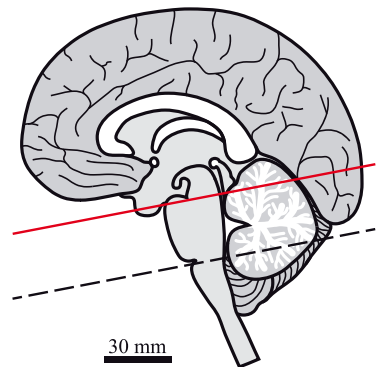

$-27.14 \mathrm{~mm}$ 


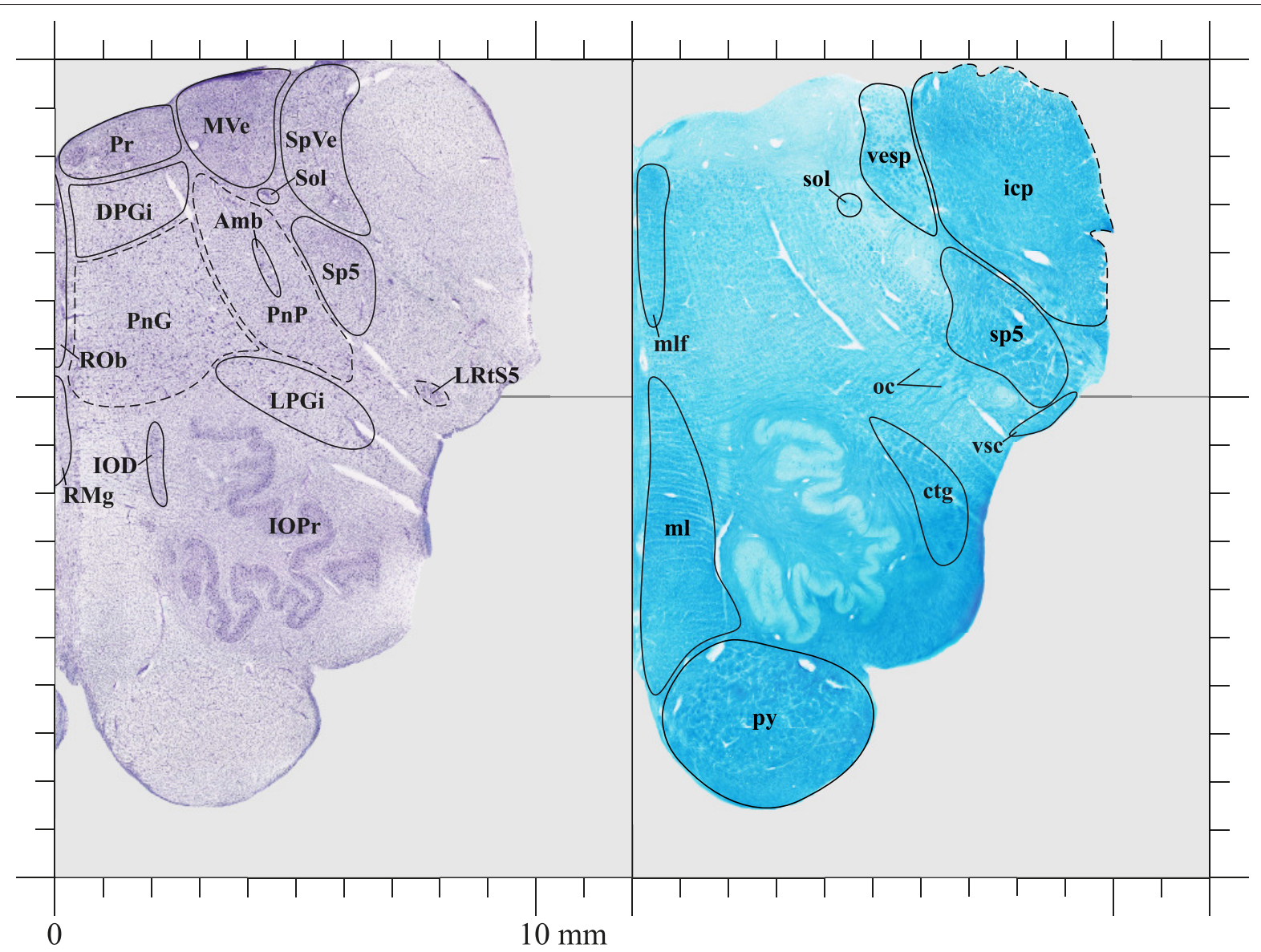

Amb: Ambiguus nucleus

DPGi : Dorsal paragigantocellular nucleus

IOD : Inferior olive, dorsal nucleus

IOPr : Inferior olive, principal nucleus

LPGi : Lateral paragigantocellular nucleus

LRtS5 : Lateral reticular nucleus, subtrigeminal part

MVe: Medial vestibular nucleus

PnG : Pontine reticular nucleus, gigantocellular part

PnP : Pontine reticular nucleus, parvocellular part

Pr: Prepositus nucleus

$\mathrm{RMg}$ : Raphe magnus nucleus

$\mathrm{ROb}$ : Raphe obscurus nucleus

Sol : Solitary nucleus

Sp5 : $\quad$ Spinal trigeminal nucleus

SpVe : Spinal vestibular nucleus

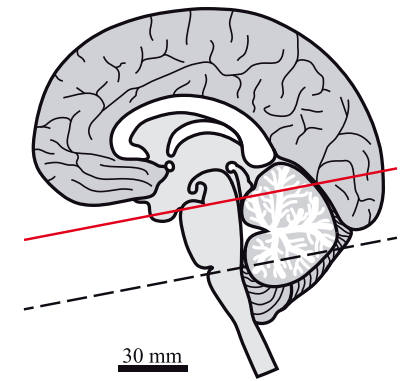

ctg : Central tegmental tract

icp : Inferior cerebellar peduncle

$\mathrm{ml}$ : $\quad$ Medial lemniscus

mlf : Medial longitudinal fasciculus

py : $\quad$ Pyramidal tract

oc : Olivocerebellar tract

sol : Solitary tract

sp5: Spinal trigeminal tract

vesp : Vestibulospinal tract

vsc : Ventral spinocerebellar tract 


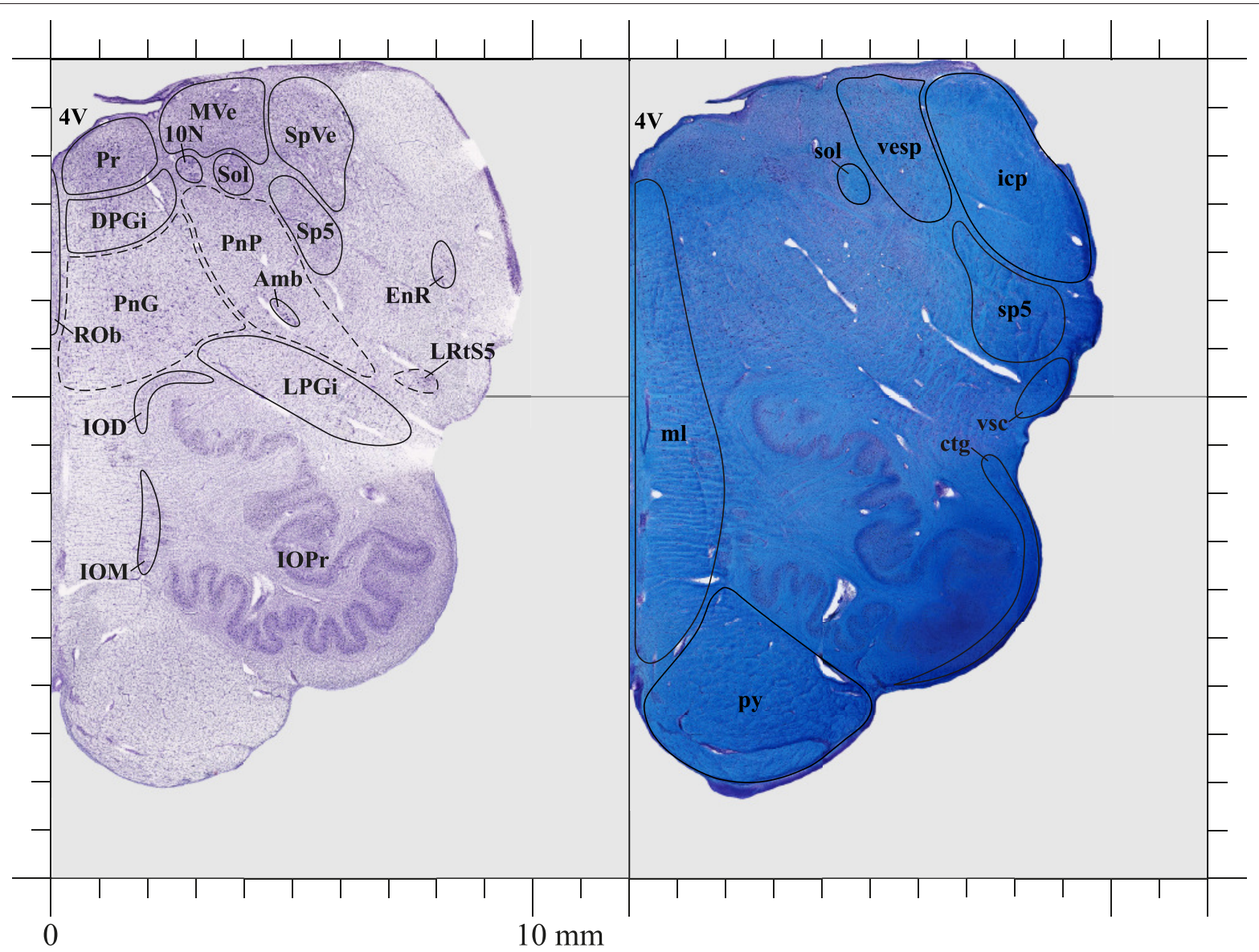

4V: $\quad$ Fourth ventricle

10N : Dorsal motor nucleus of the vagus nerve

Amb : Ambiguus nucleus

DPGi : Dorsal paragigantocellular nucleus

EnR : Endorestiform nucleus

IOD : Inferior olive, dorsal nucleus

IOM : Inferior olive, medial nucleus

IOPr : Inferior olive, principal nucleus

LPGi : Lateral paragigantocellular nucleus

LRtS5 : Lateral reticular nucleus, subtrigeminal part

MVe : Medial vestibular nucleus

PnG : Pontine reticular nucleus, gigantocellular part

PnP : Pontine reticular nucleus, parvocellular part

Pr: $\quad$ Prepositus nucleus

$\mathrm{ROb}$ : Raphe obscurus nucleus

Sol : Solitary nucleus

Sp5 : $\quad$ Spinal trigeminal nucleus

SpVe : Spinal vestibular nucleus
4V : Fourth ventricle

ctg : Central tegmental tract

icp : Inferior cerebellar peduncle

$\mathrm{ml}$ : Medial lemniscus

py : Pyramidal tract

sol : $\quad$ Solitary tract

sp5: Spinal trigeminal tract

vesp : Vestibulospinal tract

vsc : Ventral spinocerebellar tract

\section{Plate 31}

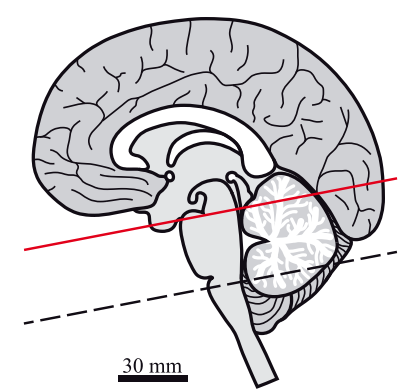




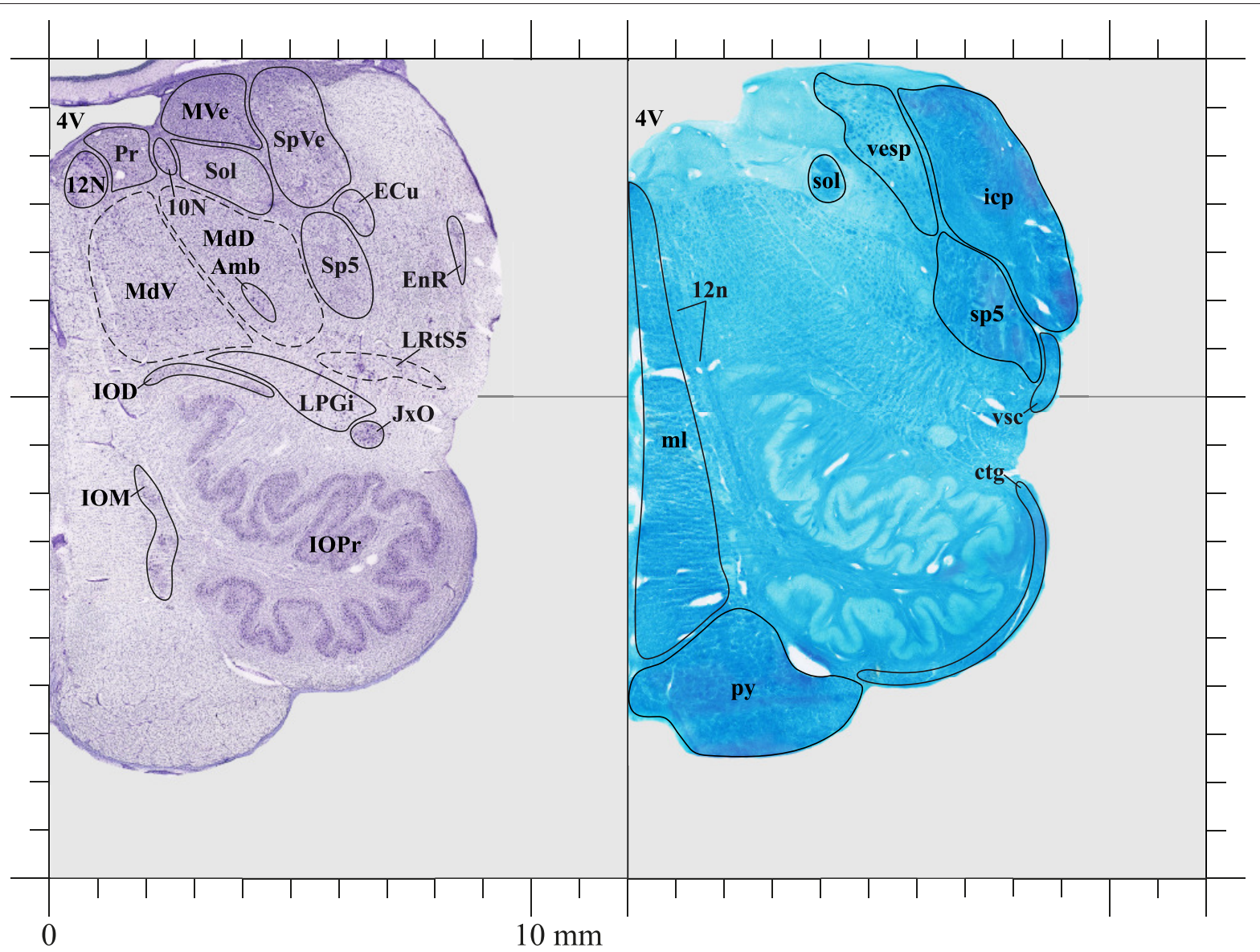

4V: $\quad$ Fourth ventricle

$10 \mathrm{~N}$ : Dorsal motor nucleus of the vagus nerve

$12 \mathrm{~N}$ : Hypoglossal nucleus

$\mathrm{Amb}$ : Ambiguus nucleus

$\mathrm{ECu}$ : External cuneate nucleus

EnR: Endorestiform nucleus

IOD : Inferior olive, dorsal nucleus

IOM : Inferior olive, medial nucleus

IOPr : Inferior olive, principal nucleus

$\mathrm{JxO}$ : Juxtaolivary nucleus

LPGi : Lateral paragigantocellular nucleus

LRtS5 : Lateral reticular nucleus, subtrigeminal part

MdD : Medullary reticular nucleus, dorsal part

MdV : Medullary reticular nucleus, ventral part

MVe: Medial vestibular nucleus

Pr : Prepositus nucleus

Sol: $\quad$ Solitary nucleus

Sp5 : $\quad$ Spinal trigeminal nucleus

SpVe: Spinal vestibular nucleus
Plate 32

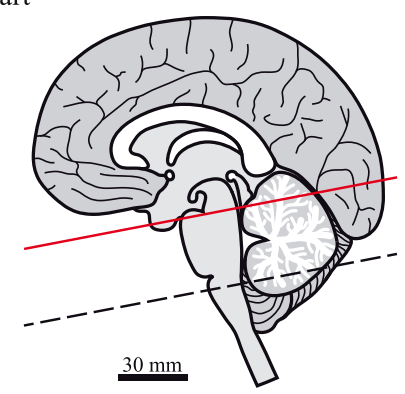

4V : Fourth ventricle

12n : Hypoglossal nerve

ctg : Central tegmental tract

icp : Inferior cerebellar peduncle

$\mathrm{ml}$ : $\quad$ Medial lemniscus

py : Pyramidal tract

sol: $\quad$ Solitary tract

sp5: $\quad$ Spinal trigeminal tract

vesp : Vestibulospinal tract

vsc : Ventral spinocerebellar tract 


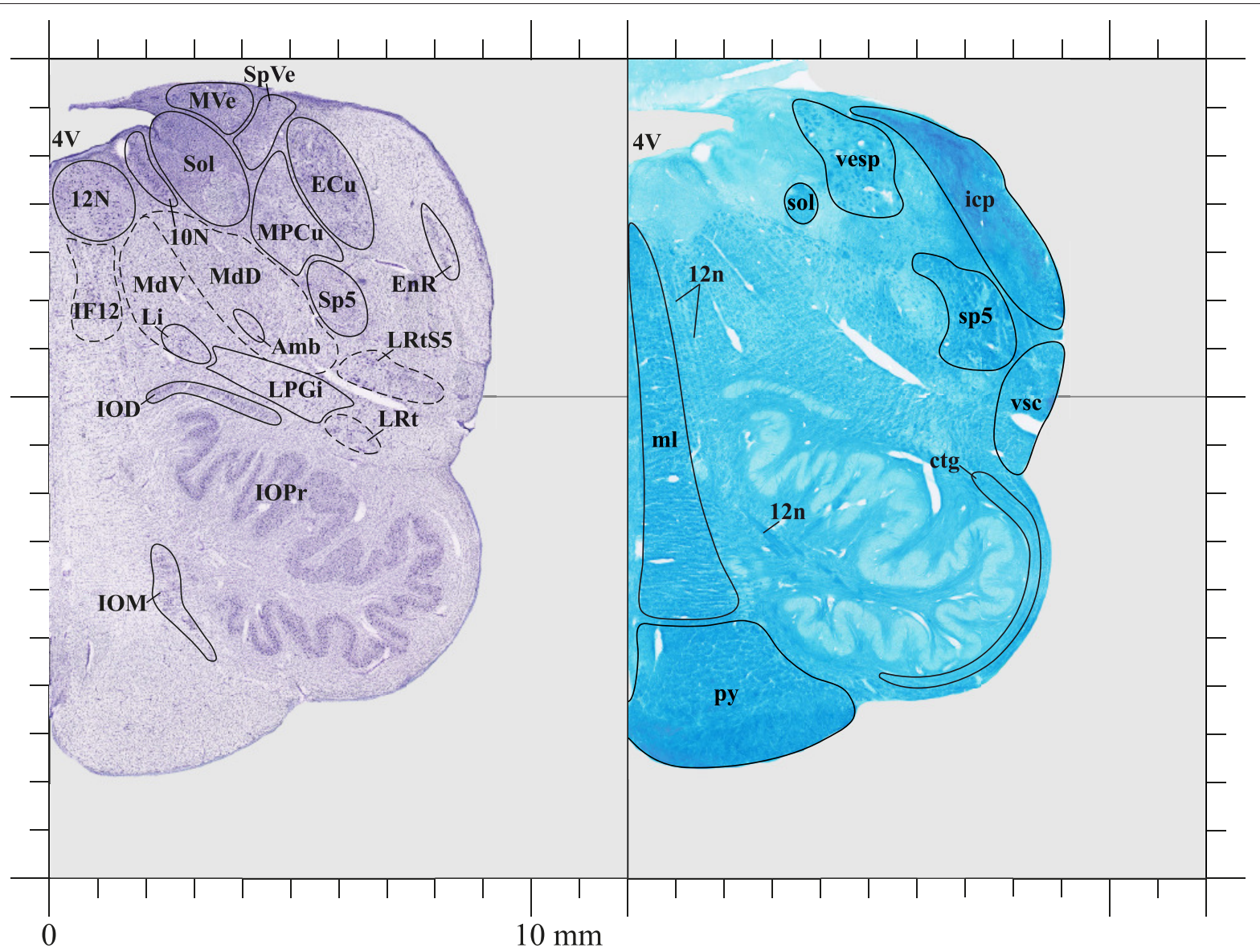

4V: $\quad$ Fourth ventricle

$10 \mathrm{~N}$ : Dorsal motor nucleus of the vagus nerve

$12 \mathrm{~N}$ : Hypoglossal nucleus

Amb : Ambiguus nucleus

$\mathrm{ECu}$ : External cuneate nucleus

EnR: Endorestiform nucleus

IF12: Interfascicular nucleus of the hypoglossal nerve

IOD : Inferior olive, dorsal nucleus

IOM : Inferior olive, medial nucleus

IOPr : Inferior olive, principal nucleus

Li : Linear nucleus of the hindbrain

LPGi : Lateral paragigantocellular nucleus

LRt : Lateral reticular nucleus

LRtS5 : Lateral reticular nucleus, subtrigeminal part

MdD : Medullary reticular nucleus, dorsal part

MdV : Medullary reticular nucleus, ventral part

$\mathrm{MPCu}$ : Medial pericuneate nucleus

MVe : Medial vestibular nucleus

Sol : $\quad$ Solitary nucleus

Sp5 : $\quad$ Spinal trigeminal nucleus

SpVe : Spinal vestibular nucleus

\section{Plate 33}

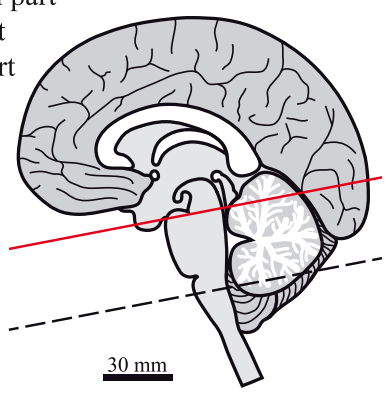

4V : $\quad$ Fourth ventricle

12n : Hypoglossal nerve

ctg : Central tegmental tract

icp : Inferior cerebellar peduncle

$\mathrm{ml}$ : Medial lemniscus

py : Pyramidal tract

sol: $\quad$ Solitary tract

sp5: $\quad$ Spinal trigeminal tract

vesp : Vestibulospinal tract

vsc : Ventral spinocerebellar tract 


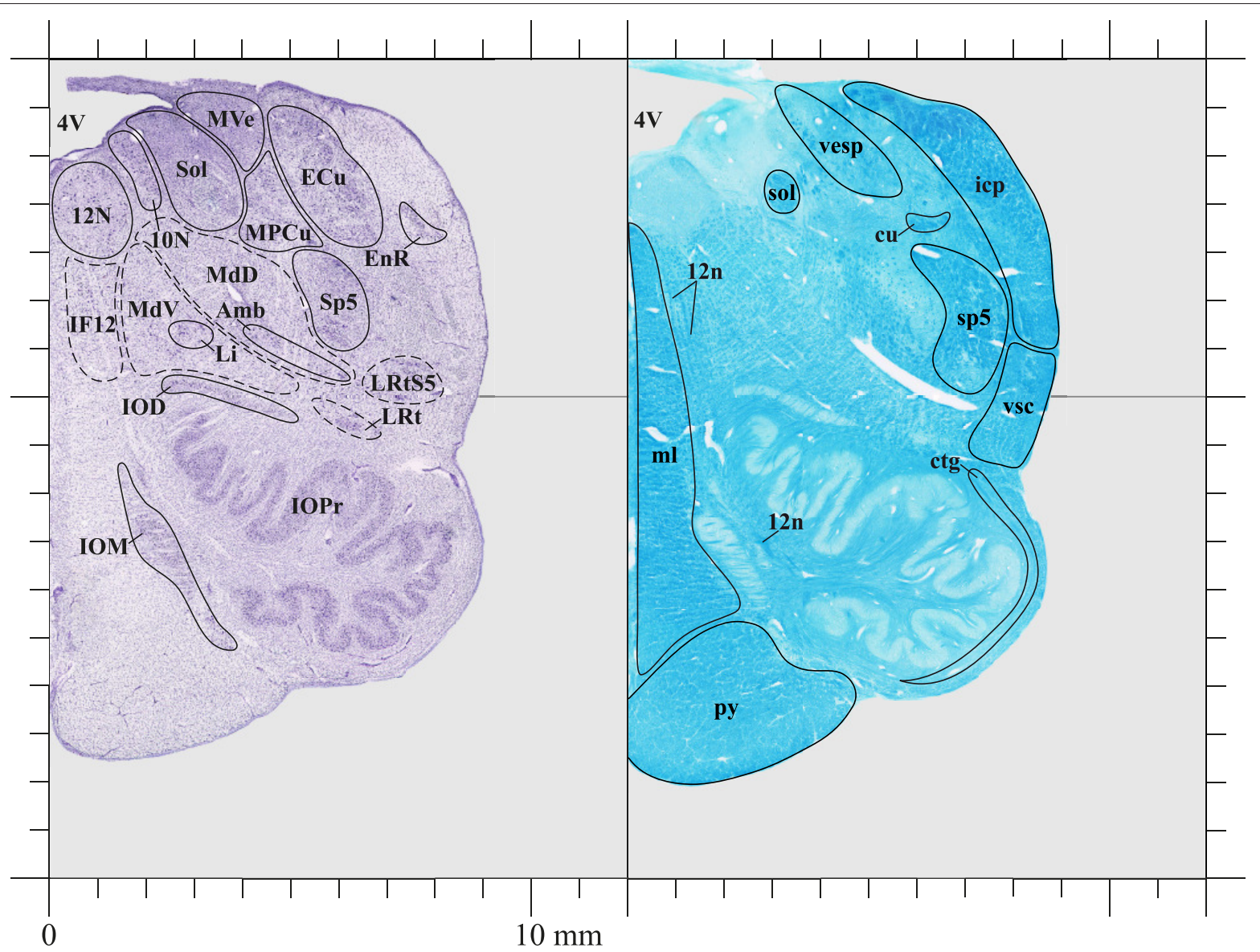

4V: $\quad$ Fourth ventricle

$10 \mathrm{~N}$ : Dorsal motor nucleus of the vagus nerve

$12 \mathrm{~N}$ : Hypoglossal nucleus

Amb : Ambiguus nucleus

$\mathrm{ECu}$ : External cuneate nucleus

EnR: Endorestiform nucleus

IF12: Interfascicular nucleus of the hypoglossal nerve

IOD : Inferior olive, dorsal nucleus

IOM : Inferior olive, medial nucleus

IOPr : Inferior olive, principal nucleus

Li : $\quad$ Linear nucleus of the hindbrain

LRt : Lateral reticular nucleus

LRtS5 : Lateral reticular nucleus, subtrigeminal part

MdD : Medullary reticular nucleus, dorsal part

MdV : Medullary reticular nucleus, ventral part

$\mathrm{MPCu}$ : Medial pericuneate nucleus

MVe : Medial vestibular nucleus

Sol : $\quad$ Solitary nucleus

Sp5 : $\quad$ Spinal trigeminal nucleus
Plate 34

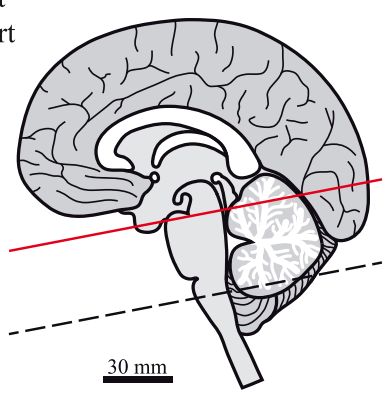

4V : $\quad$ Fourth ventricle

12n : Hypoglossal nerve

ctg : Central tegmental tract

cu : Cuneate fasciculus

icp : Inferior cerebellar peduncle

$\mathrm{ml}$ : Medial lemniscus

py : Pyramidal tract

sol: $\quad$ Solitary tract

sp5: Spinal trigeminal tract

vesp : Vestibulospinal tract

vsc : Ventral spinocerebellar tract 


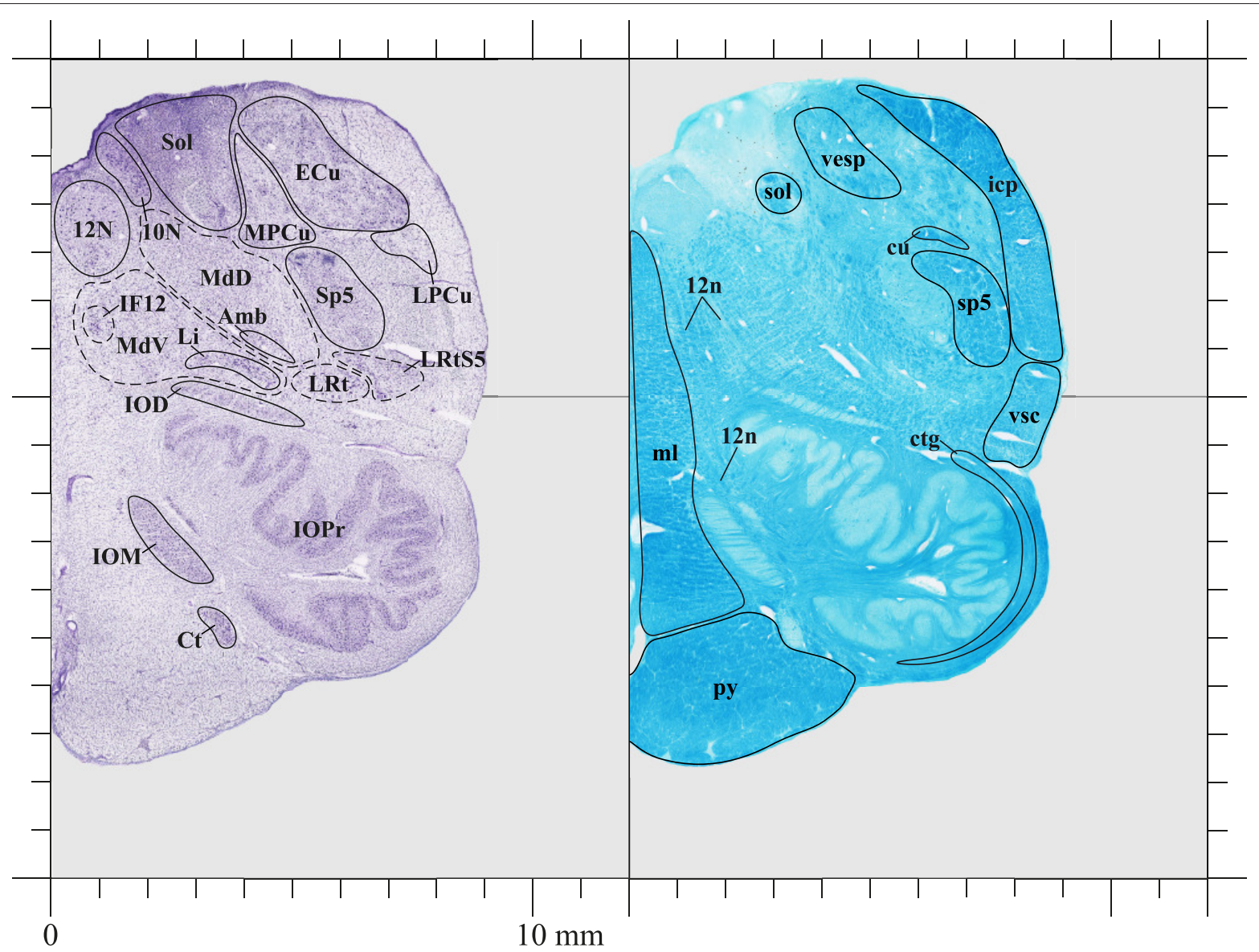

$10 \mathrm{~N}$ : Dorsal motor nucleus of the vagus nerve

12N : Hypoglossal nucleus

Amb: Ambiguus nucleus

$\mathrm{Ct}$ : Conterminal nucleus

$\mathrm{ECu}$ : External cuneate nucleus

IF12: Interfascicular nucleus of the hypoglossal nerve

IOD : Inferior olive, dorsal nucleus

IOM : Inferior olive, medial nucleus

IOPr : Inferior olive, principal nucleus

$\mathrm{Li}$ : Linear nucleus of the hindbrain

12n: Hypoglossal nerve

ctg : Central tegmental tract

cu : Cuneate fasciculus

icp : Inferior cerebellar peduncle

$\mathrm{ml}$ : $\quad$ Medial lemniscus

py : Pyramidal tract

sol : $\quad$ Solitary tract

sp5: $\quad$ Spinal trigeminal tract

$\mathrm{LPCu}$ : Lateral pericuneate nucleus

vesp : Vestibulospinal tract

LRt : Lateral reticular nucleus

LRtS5 : Lateral reticular nucleus, subtrigeminal part

MdD : Medullary reticular nucleus, dorsal part

MdV : Medullary reticular nucleus, ventral part

$\mathrm{MPCu}$ : Medial pericuneate nucleus

Sol : Solitary nucleus

Sp5 : $\quad$ Spinal trigeminal nucleus

\section{Plate 35}

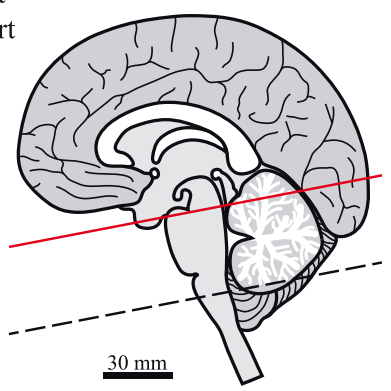




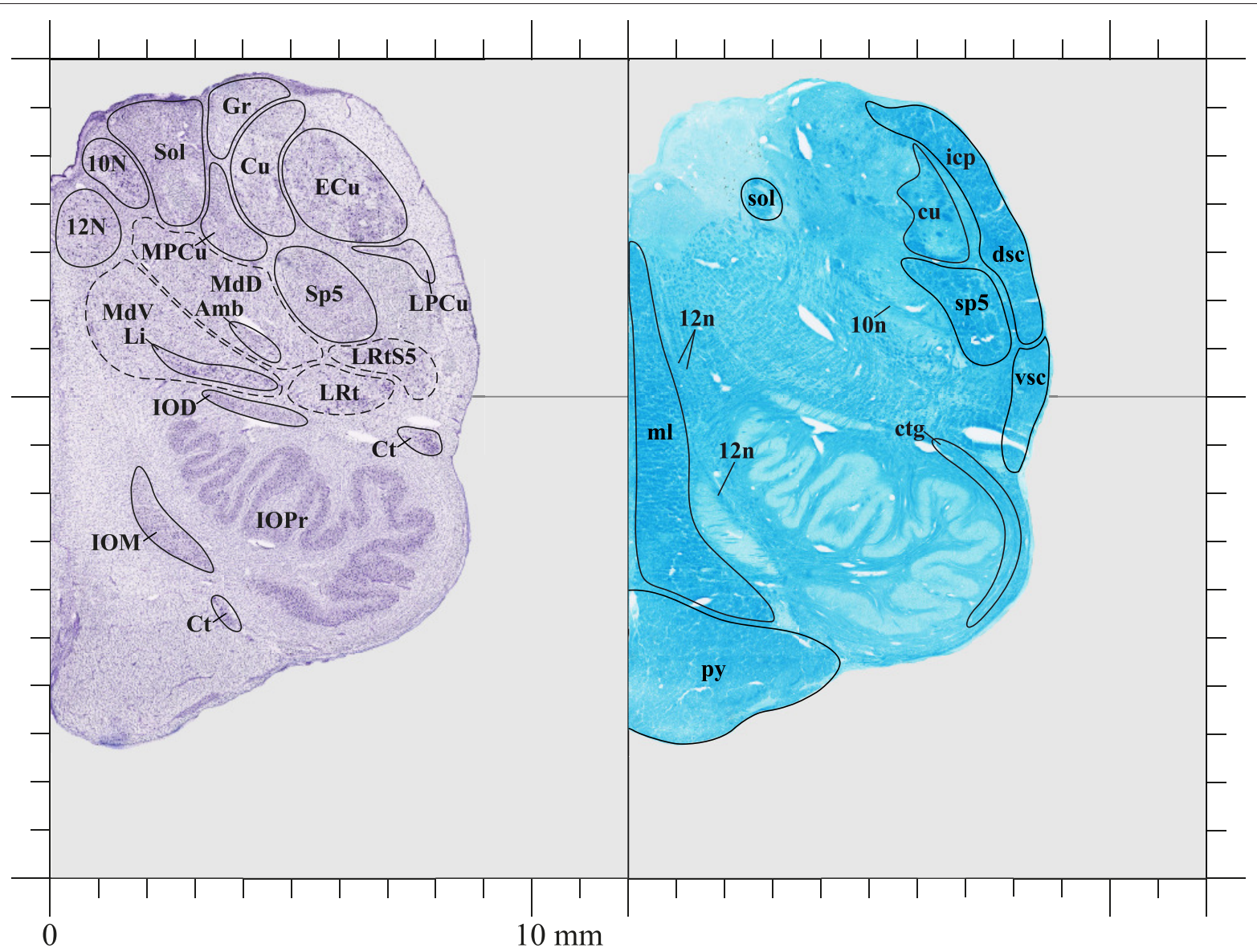

$10 \mathrm{~N}$ : Dorsal motor nucleus of the vagus nerve

12N : Hypoglossal nucleus

$\mathrm{Amb}$ : Ambiguus nucleus

$\mathrm{Ct}$ : Conterminal nucleus

$\mathrm{Cu}$ : Cuneate nucleus

$\mathrm{ECu}$ : External cuneate nucleus

$\mathrm{Gr}$ : Gracile nucleus

IOD : Inferior olive, dorsal nucleus

IOM : Inferior olive, medial nucleus

IOPr : Inferior olive, principal nucleus

10n: Vagus nerve

12n: Hypoglossal nerve

ctg : Central tegmental tract

$\mathrm{cu}$ : Cuneate fasciculus

dsc : Dorsal spinocerebellar tract

icp : Inferior cerebellar peduncle

$\mathrm{ml}$ : $\quad$ Medial lemniscus

py : Pyramidal tract

sol : $\quad$ Solitary tract

Li : $\quad$ Linear nucleus of the hindbrain

sp5: Spinal trigeminal tract

$\mathrm{LPCu}$ : Lateral pericuneate nucleus

vsc : Ventral spinocerebellar tract

LRt : Lateral reticular nucleus

LRtS5 : Lateral reticular nucleus, subtrigeminal part

MdD : Medullary reticular nucleus, dorsal part

MdV : Medullary reticular nucleus, ventral part

$\mathrm{MPCu}$ : Medial pericuneate nucleus

Sol : $\quad$ Solitary nucleus

Sp5 : $\quad$ Spinal trigeminal nucleus

\section{Plate 36}

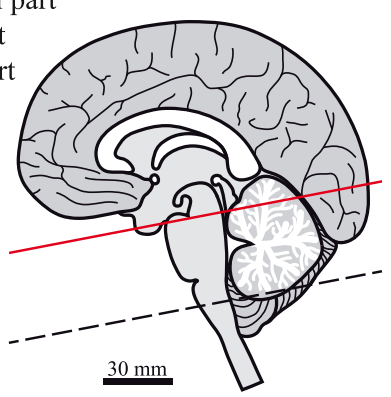




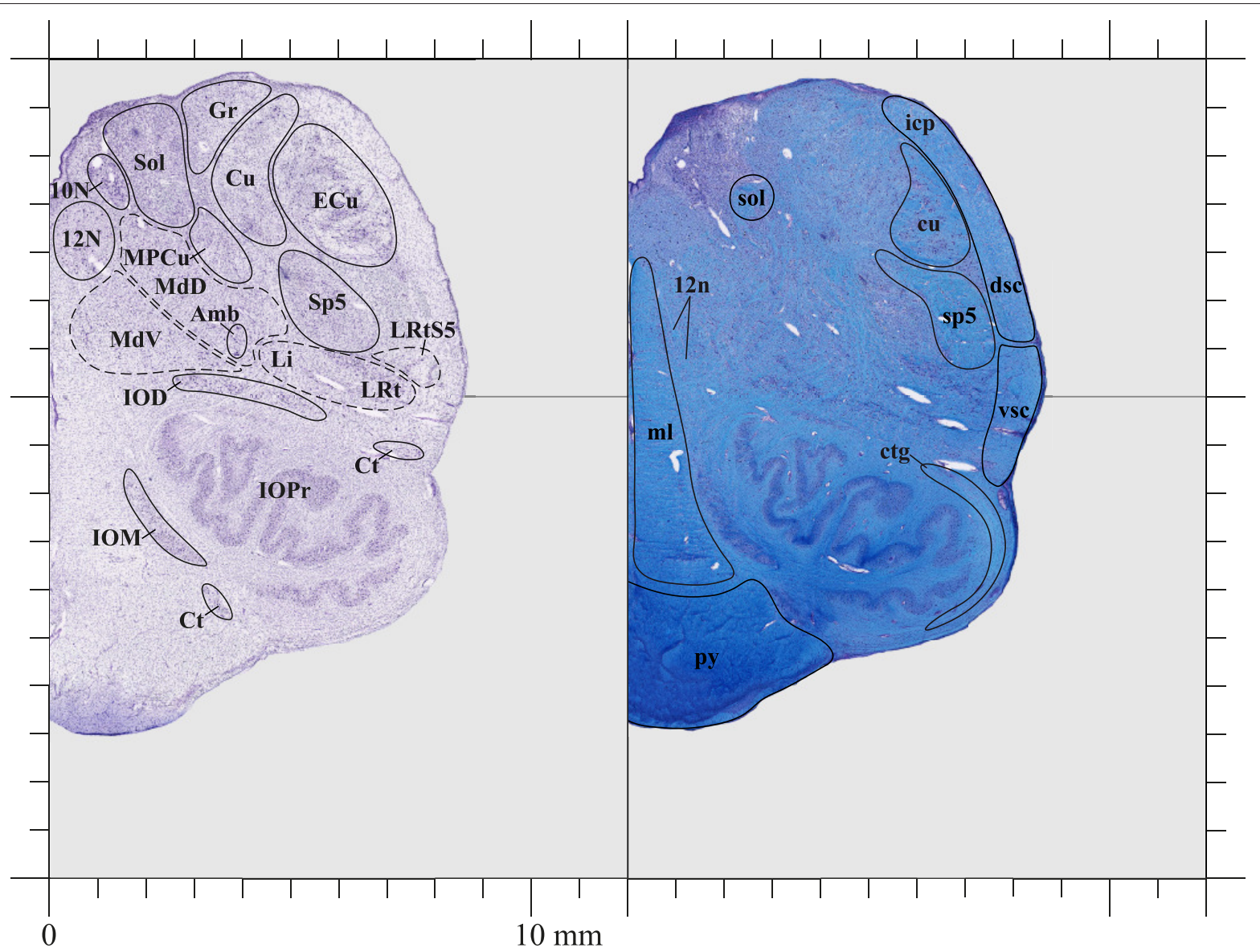

$10 \mathrm{~N}$ : Dorsal motor nucleus of the vagus nerve

12N : Hypoglossal nucleus

Amb: Ambiguus nucleus

$\mathrm{Ct}$ : Conterminal nucleus

$\mathrm{Cu}$ : Cuneate nucleus

$\mathrm{ECu}$ : External cuneate nucleus

$\mathrm{Gr}$ : Gracile nucleus

IOD : Inferior olive, dorsal nucleus

IOM : Inferior olive, medial nucleus

IOPr : Inferior olive, principal nucleus

12n: Hypoglossal nerve

ctg : Central tegmental tract

cu : Cuneate fasciculus

dsc : Dorsal spinocerebellar tract

icp : Inferior cerebellar peduncle

$\mathrm{ml}$ : $\quad$ Medial lemniscus

py : Pyramidal tract

sol: $\quad$ Solitary tract

$\mathrm{Li}$ : $\quad$ Linear nucleus of the hindbrain

sp5: $\quad$ Spinal trigeminal tract

LRt : Lateral reticular nucleus

LRtS5 : Lateral reticular nucleus, subtrigeminal part

MdD : Medullary reticular nucleus, dorsal part

MdV : Medullary reticular nucleus, ventral part

$\mathrm{MPCu}$ : Medial pericuneate nucleus

Sol : Solitary nucleus

Sp5 : Spinal trigeminal nucleus

\section{Plate 37}

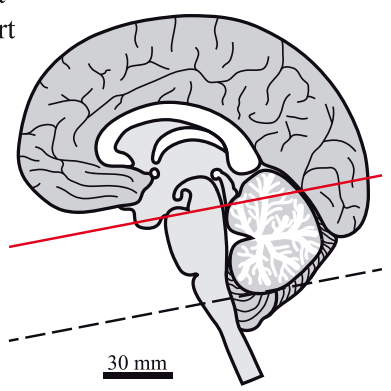




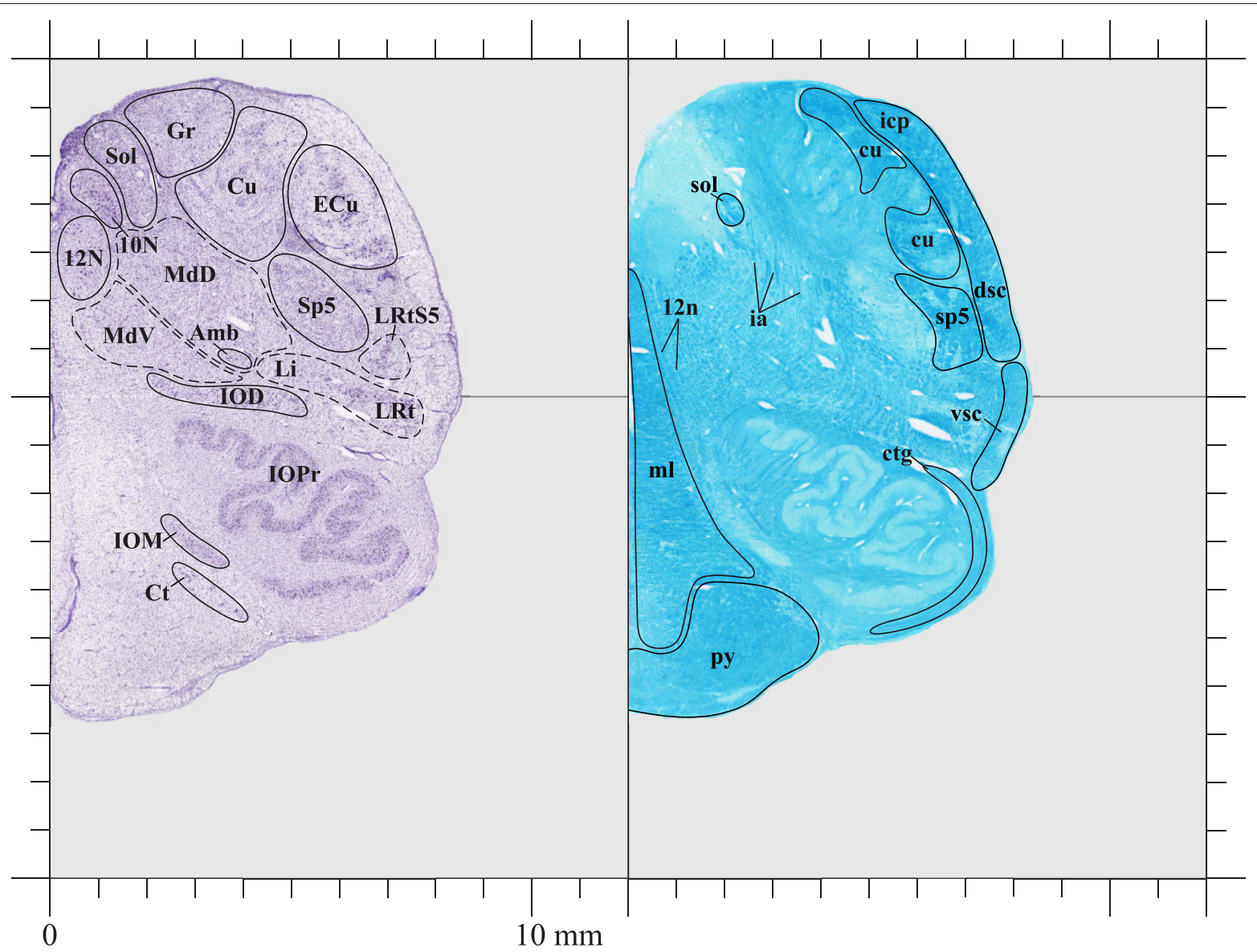

$10 \mathrm{~N}$ : Dorsal motor nucleus of the vagus nerve

12N : Hypoglossal nucleus

Amb: Ambiguus nucleus

$\mathrm{Ct}$ : Conterminal nucleus

$\mathrm{Cu}$ : Cuneate nucleus

$\mathrm{ECu}$ : External cuneate nucleus

$\mathrm{Gr}$ : Gracile nucleus

IOD : Inferior olive, dorsal nucleus

IOM : Inferior olive, medial nucleus

IOPr : Inferior olive, principal nucleus

Li : $\quad$ Linear nucleus of the hindbrain

12n: Hypoglossal nerve

ctg : Central tegmental tract

cu : Cuneate fasciculus

dsc : Dorsal spinocerebellar tract

ia : Internal arcuate fibers

icp : Inferior cerebellar peduncle

$\mathrm{ml}$ : $\quad$ Medial lemniscus

py : Pyramidal tract

sol : $\quad$ Solitary tract

sp5 : $\quad$ Spinal trigeminal tract

LRt : Lateral reticular nucleus

vsc : Ventral spinocerebellar tract

LRtS5 : Lateral reticular nucleus, subtrigeminal part

MdD : Medullary reticular nucleus, dorsal part

MdV : Medullary reticular nucleus, ventral part

Sol : Solitary nucleus

Sp5 : Spinal trigeminal nucleus

\section{Plate 38}

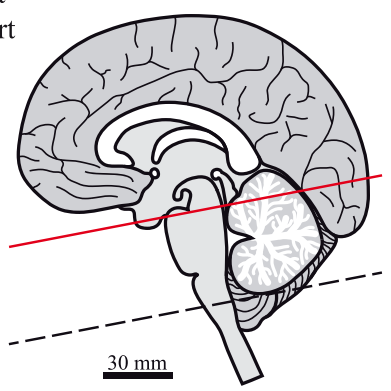




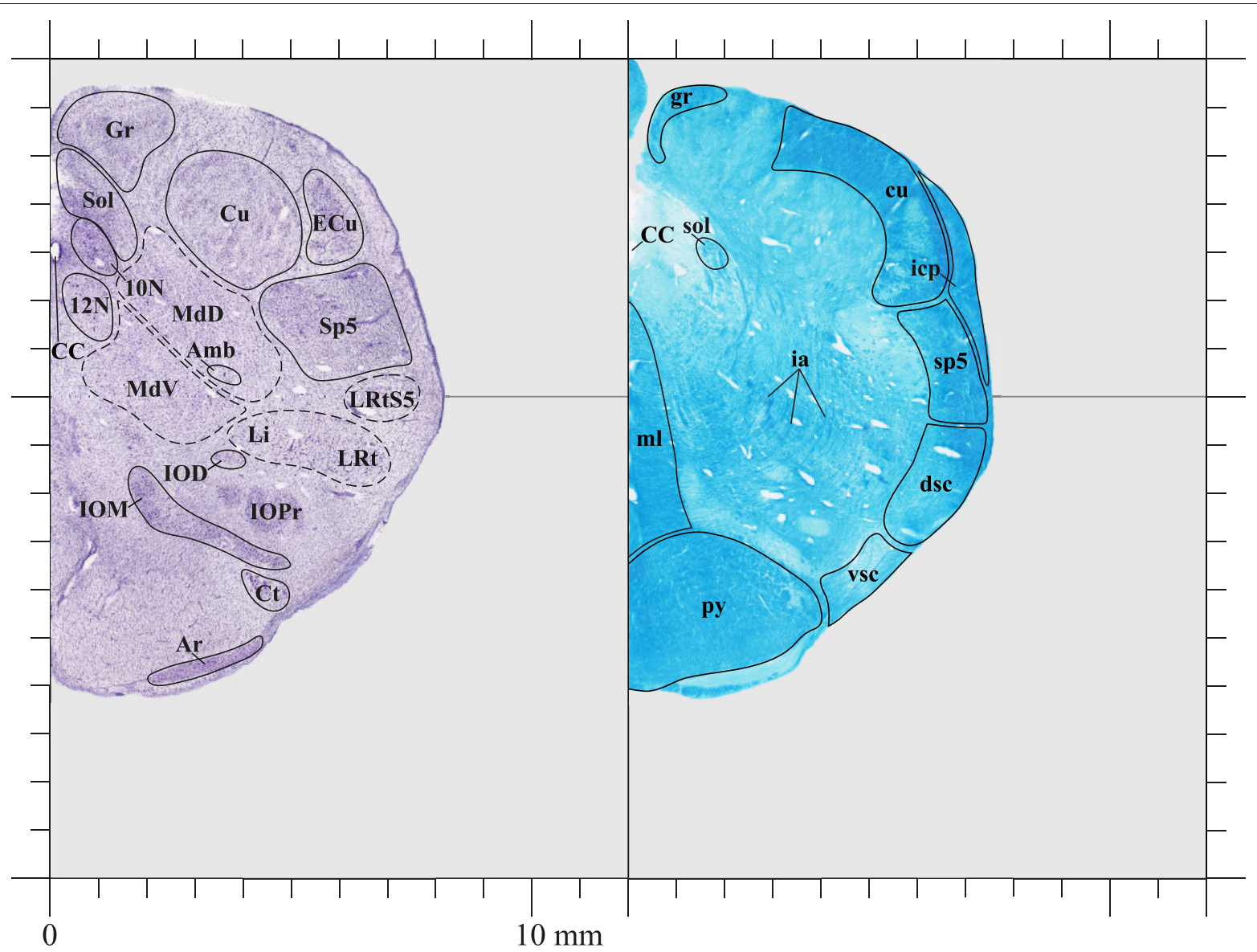

$10 \mathrm{~N}$ : Dorsal motor nucleus of the vagus nerve

12N : Hypoglossal nucleus

Amb: Ambiguus nucleus

Ar: Arcuate nucleus

CC : Central canal

$\mathrm{Ct}$ : Conterminal nucleus

$\mathrm{Cu}$ : Cuneate nucleus

$\mathrm{ECu}$ : External cuneate nucleus

Gr: Gracile nucleus

IOD : Inferior olive, dorsal nucleus

IOM : Inferior olive, medial nucleus

CC : Central canal

$\mathrm{cu}$ : Cuneate fasciculus

dsc : Dorsal spinocerebellar tract

gr : Gracile fasciculus

ia : Internal arcuate fibers

IOPr : Inferior olive, principal nucleus

icp : Inferior cerebellar peduncle

$\mathrm{ml}$ : $\quad$ Medial lemniscus

py : Pyramidal tract

sol : $\quad$ Solitary tract

sp5 : $\quad$ Spinal trigeminal tract

Li : Linear nucleus of the hindbrain

LRt : Lateral reticular nucleus

LRtS5 : Lateral reticular nucleus, subtrigeminal part

MdD : Medullary reticular nucleus, dorsal part

$\mathrm{MdV}$ : Medullary reticular nucleus, ventral part

Sol : $\quad$ Solitary nucleus

Sp5 : $\quad$ Spinal trigeminal nucleus

\section{Plate 39}

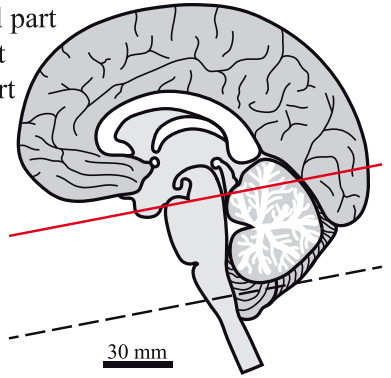




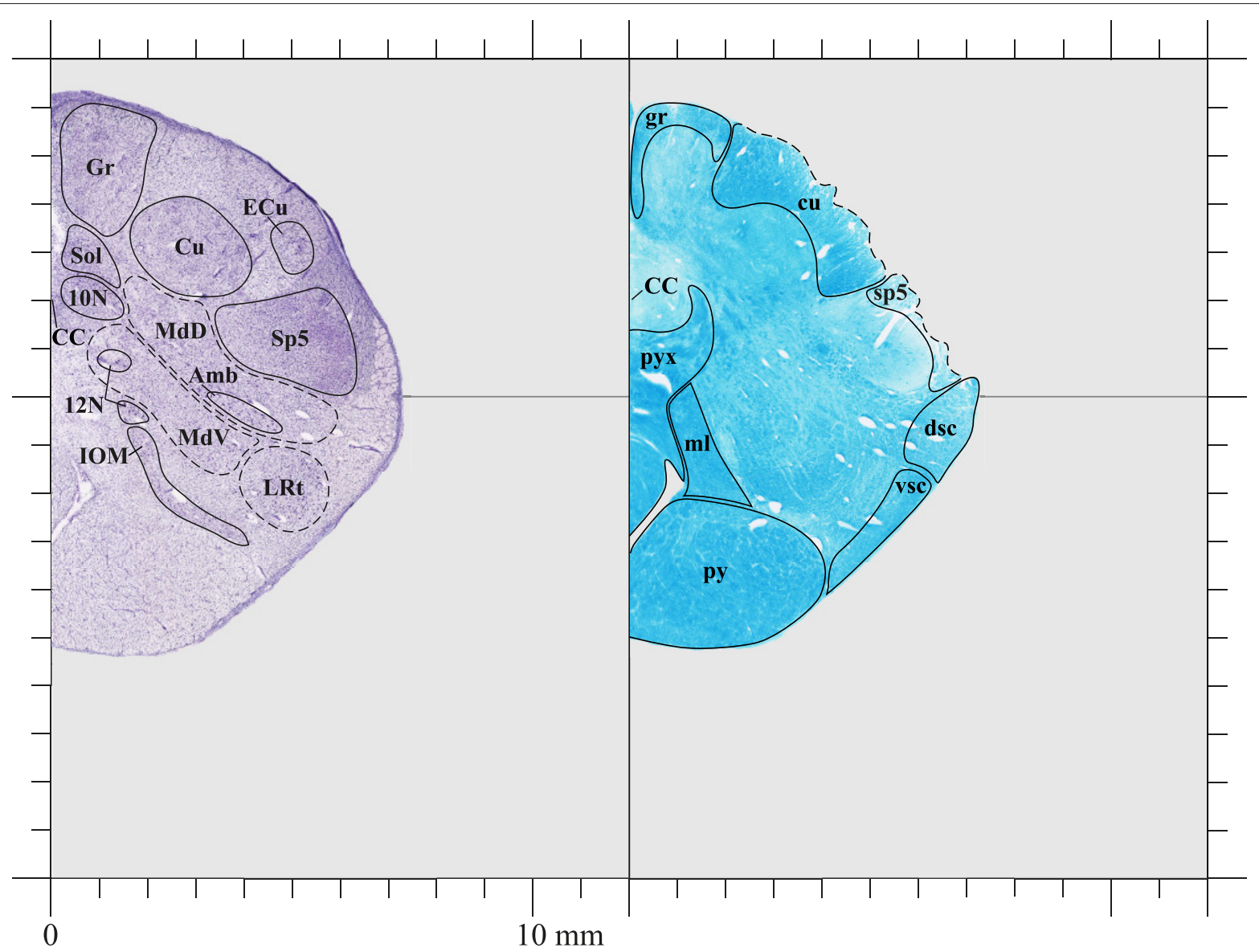

$10 \mathrm{~N}$ : Dorsal motor nucleus of the vagus nerve

12N : Hypoglossal nucleus

Amb: Ambiguus nucleus

CC : Central canal

$\mathrm{Cu}$ : Cuneate nucleus

$\mathrm{ECu}$ : External cuneate nucleus

$\mathrm{Gr}$ : Gracile nucleus

IOM : Inferior olive, medial nucleus

LRt : Lateral reticular nucleus

CC : Central canal

$\mathrm{cu}$ : Cuneate fasciculus

dsc : Dorsal spinocerebellar tract

gr : Gracile fasciculus

$\mathrm{ml}$ : $\quad$ Medial lemniscus

py : $\quad$ Pyramidal tract

pyx : Pyramidal decussation

sp5: $\quad$ Spinal trigeminal tract

MdD : Medullary reticular nucleus, dorsal part

vsc : Ventral spinocerebellar tract

MdV : Medullary reticular nucleus, ventral part

Sol : $\quad$ Solitary nucleus

Sp5 : Spinal trigeminal nucleus

\section{Plate 40}

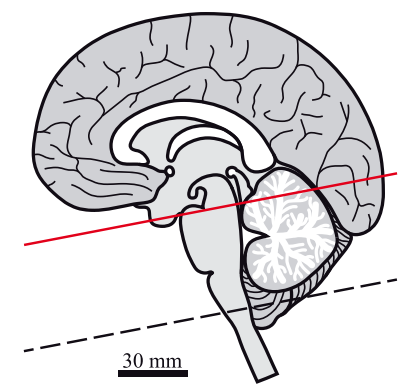




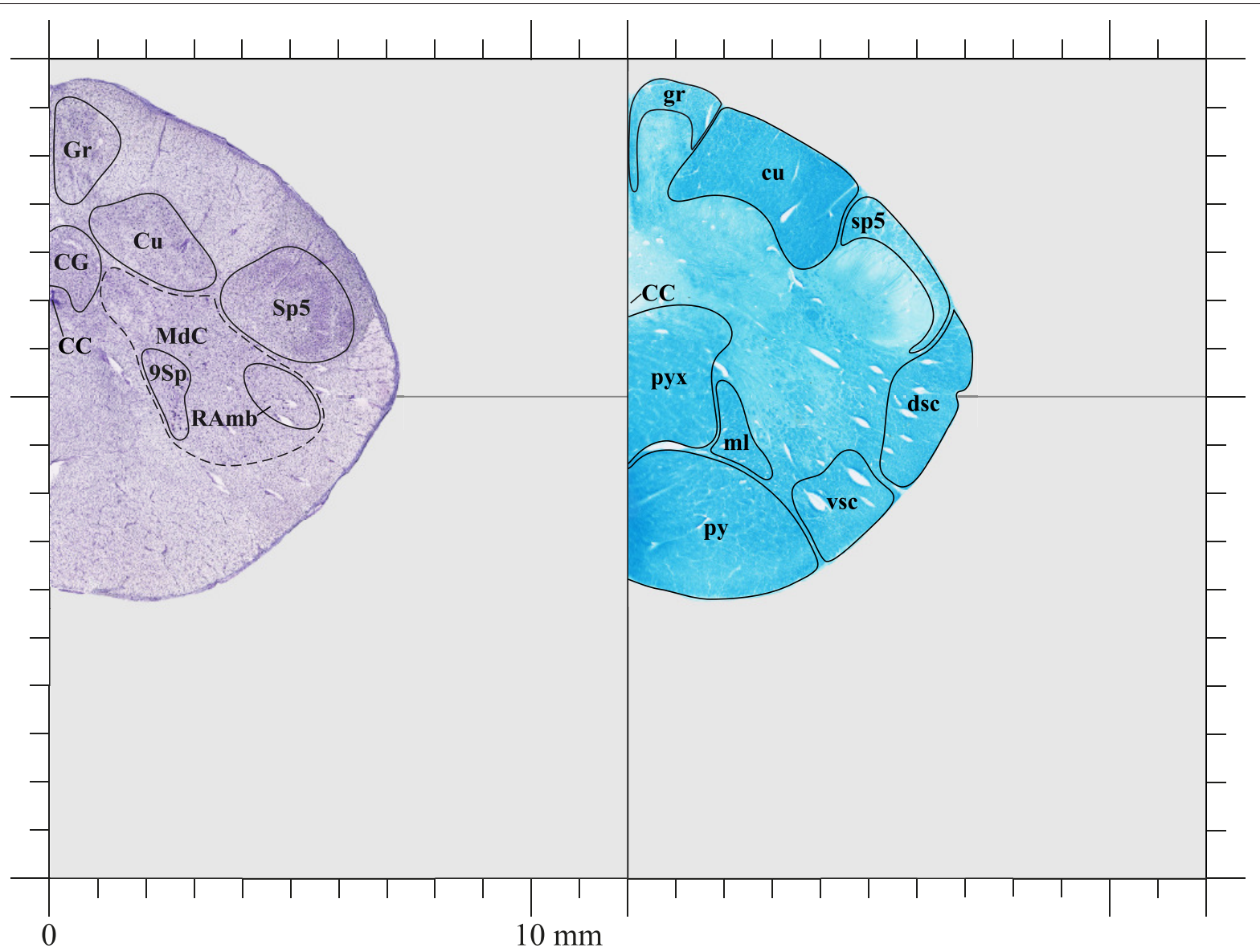

9Sp : $\quad$ Lamina 9 of the spinal gray

CC : Central canal

$\mathrm{CG}$ : Central gray

$\mathrm{Cu}$ : Cuneate nucleus

Gr: Gracile nucleus

MdC : Medullary reticular nucleus, central part

RAmb: Retroambiguus nucleus

Sp5 : $\quad$ Spinal trigeminal nucleus

CC : Central canal

$\mathrm{cu}$ : Cuneate fasciculus

dsc : Dorsal spinocerebellar tract

gr : Gracile fasciculus

$\mathrm{ml}$ : $\quad$ Medial lemniscus

py : Pyramidal tract

pyx : Pyramidal decussation

sp5: $\quad$ Spinal trigeminal tract

vsc : Ventral spinocerebellar tract

\section{Plate 41}

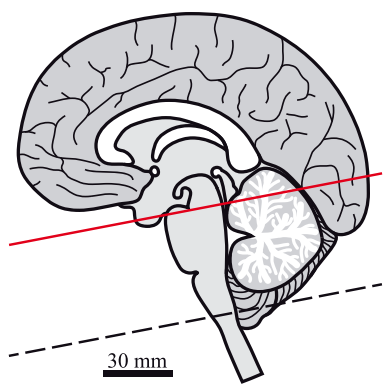




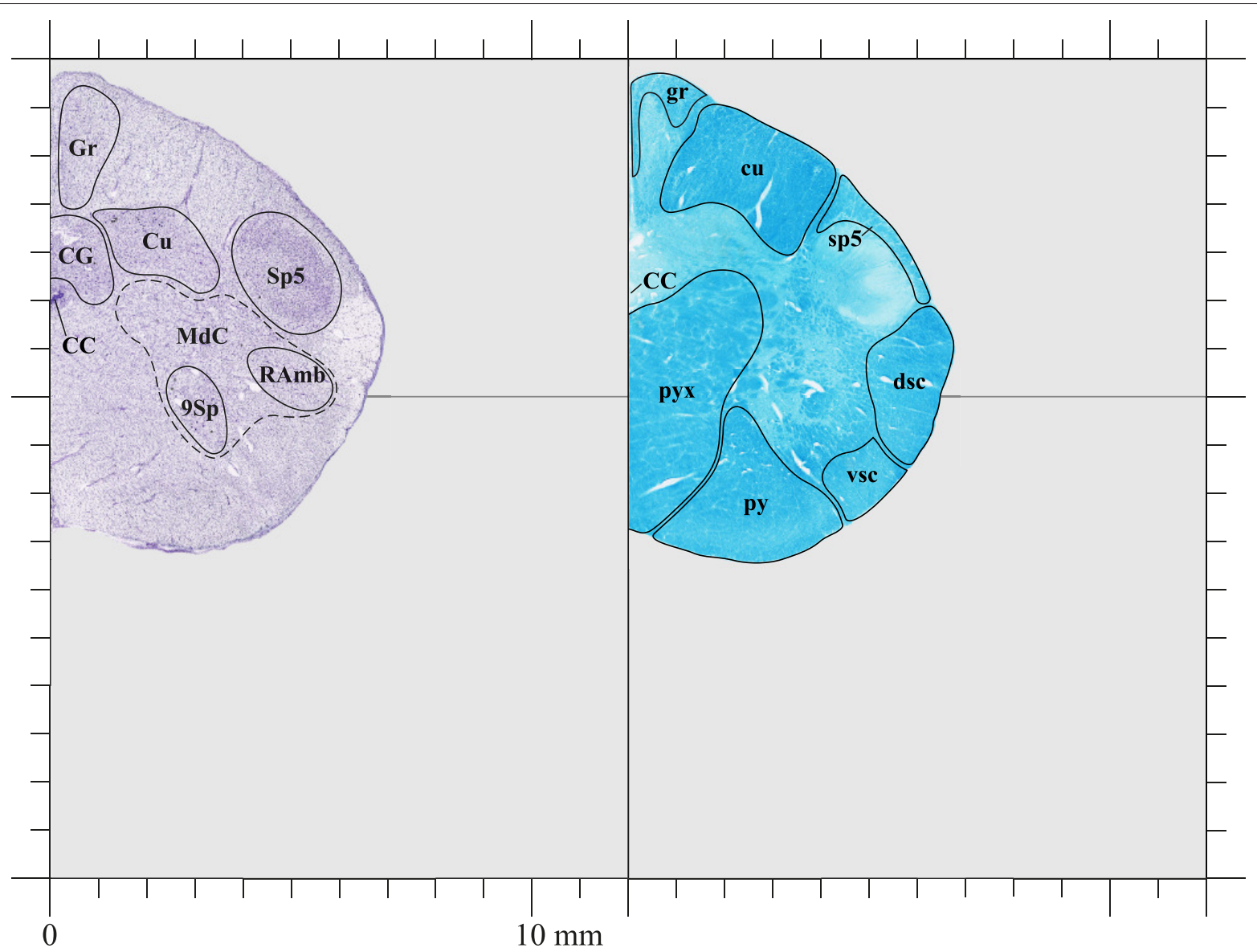

9Sp : $\quad$ Lamina 9 of the spinal gray

CC : Central canal

$\mathrm{CG}$ : Central gray

$\mathrm{Cu}$ : $\quad$ Cuneate nucleus

Gr: Gracile nucleus

MdC : Medullary reticular nucleus, central part

RAmb: Retroambiguus nucleus

Sp5 : $\quad$ Spinal trigeminal nucleus

CC : Central canal

$\mathrm{cu}$ : Cuneate fasciculus

dsc : Dorsal spinocerebellar tract

gr : Gracile fasciculus

py : $\quad$ Pyramidal tract

pyx : Pyramidal decussation

sp5 : $\quad$ Spinal trigeminal tract

vsc : Ventral spinocerebellar tract

\section{Plate 42}

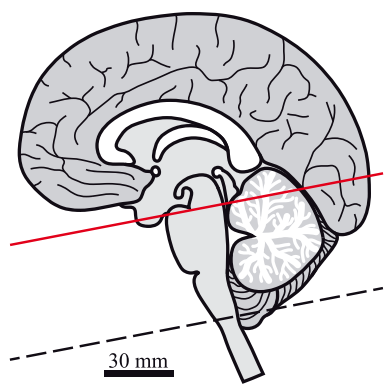




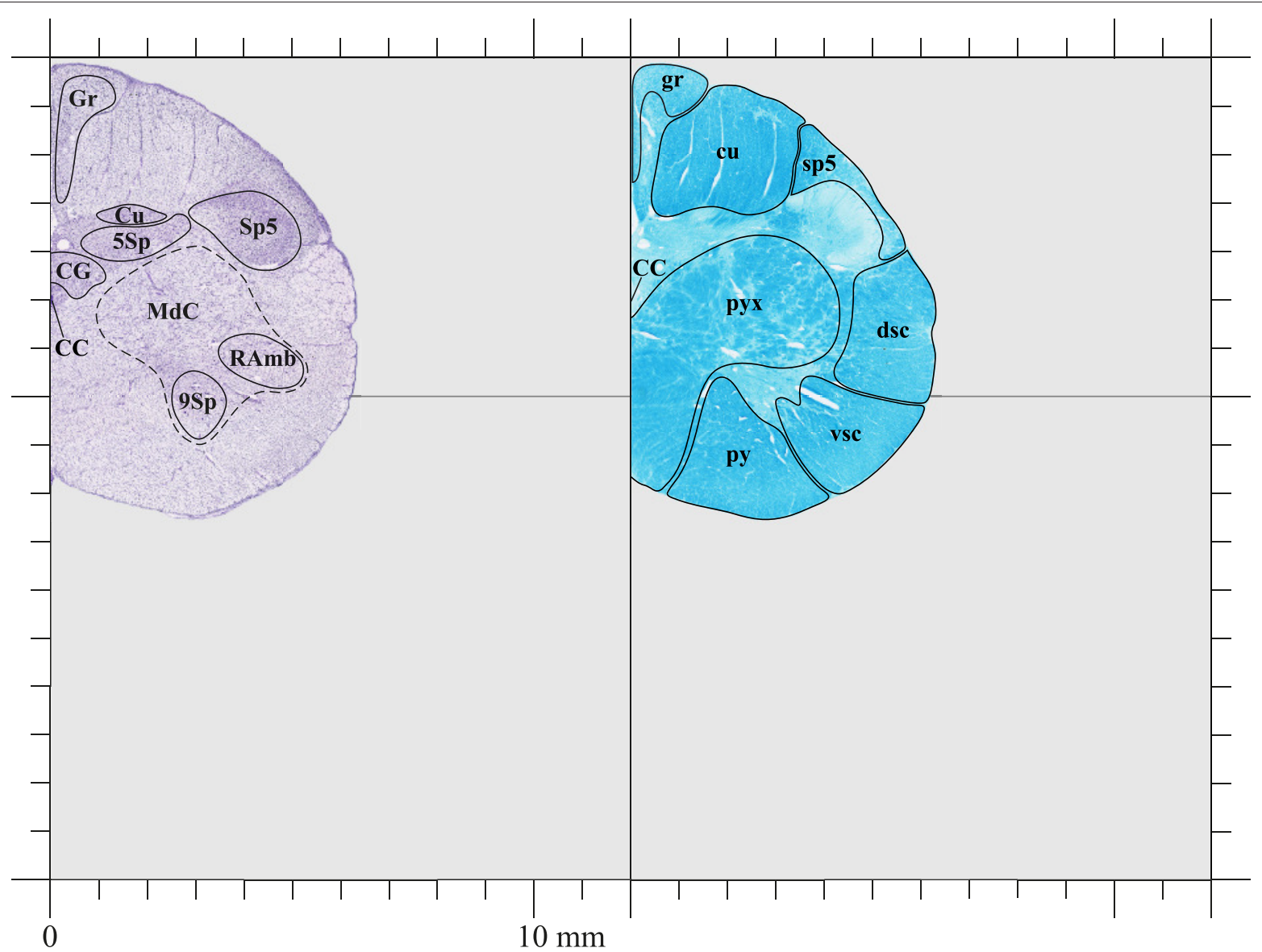

5Sp : Lamina 5 of the spinal gray

9Sp : Lamina 9 of the spinal gray

CC : Central canal

CC : Central canal

CG : Central gray

$\mathrm{Cu}$ : $\quad$ Cuneate nucleus

$\mathrm{Gr}$ : Gracile nucleus

MdC : Medullary reticular nucleus, central part

RAmb : Retroambiguus nucleus

cu : Cuneate fasciculus

dsc : Dorsal spinocerebellar tract

gr : Gracile fasciculus

py : Pyramidal tract

pyx : Pyramidal decussation

sp5 : $\quad$ Spinal trigeminal tract

Sp5 : $\quad$ Spinal trigeminal nucleus

vsc : Ventral spinocerebellar tract

\section{Plate 43}

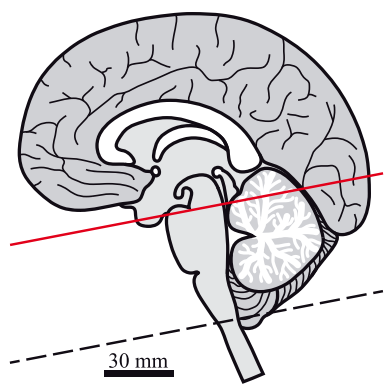




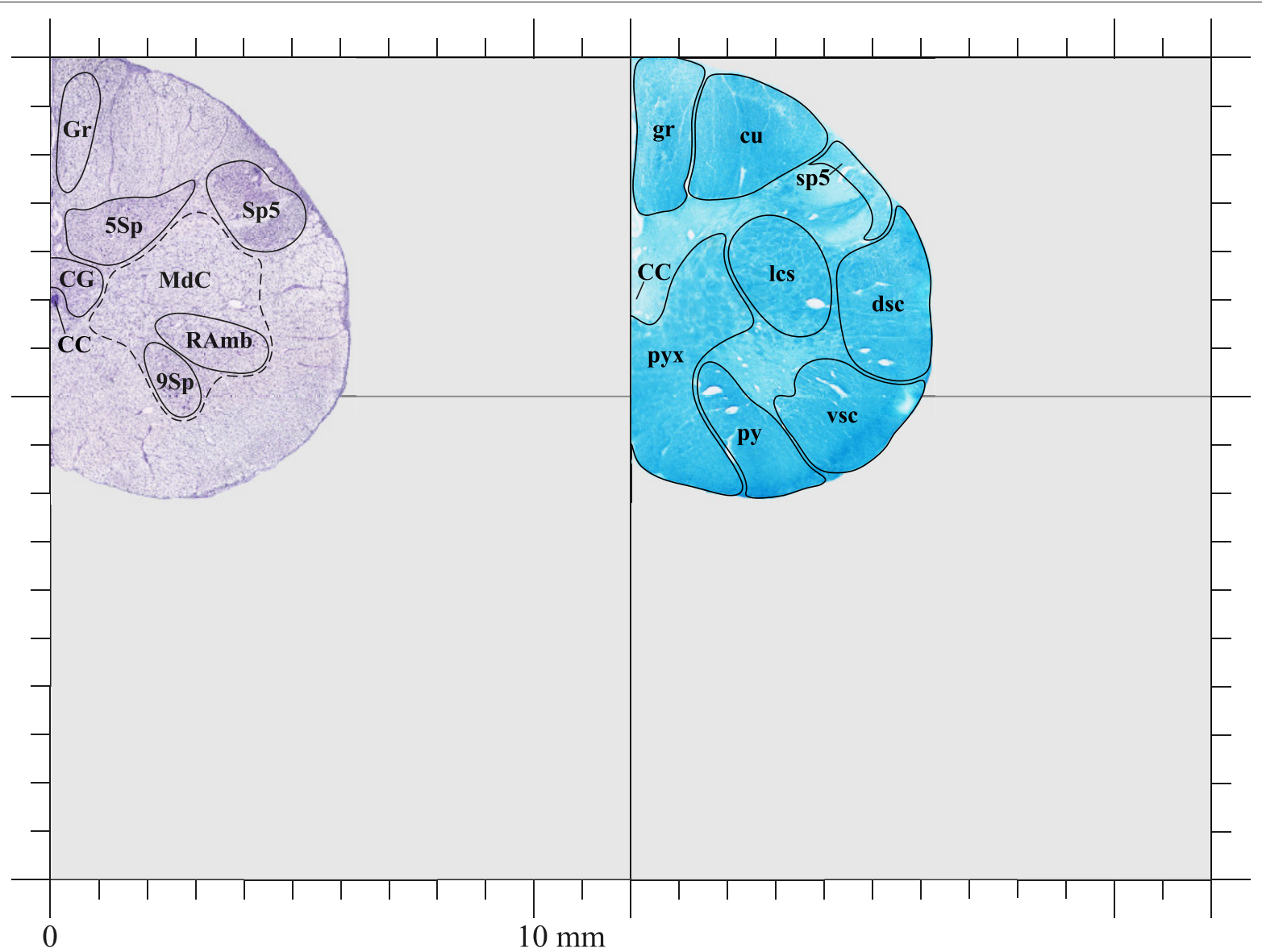

5Sp : Lamina 5 of the spinal gray

9Sp: Lamina 9 of the spinal gray

CC : Central canal

CC : Central canal

CG : Central gray

Gr: Gracile nucleus

MdC : Medullary reticular nucleus, central part

RAmb : Retroambiguus nucleus

Sp5 : $\quad$ Spinal trigeminal nucleus

$\mathrm{cu}$ : Cuneate fasciculus

dsc : Dorsal spinocerebellar tract

gr : Gracile fasciculus

lcs : Lateral corticospinal tract

py : Pyramidal tract

pyx : Pyramidal decussation

sp5: $\quad$ Spinal trigeminal tract

vsc : Ventral spinocerebellar tract

\section{Plate 44}

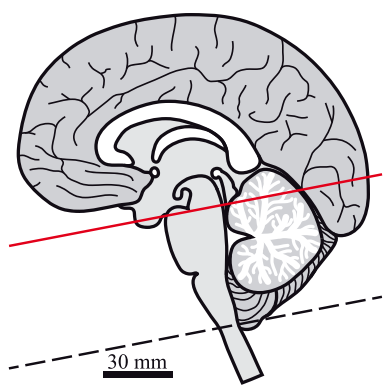




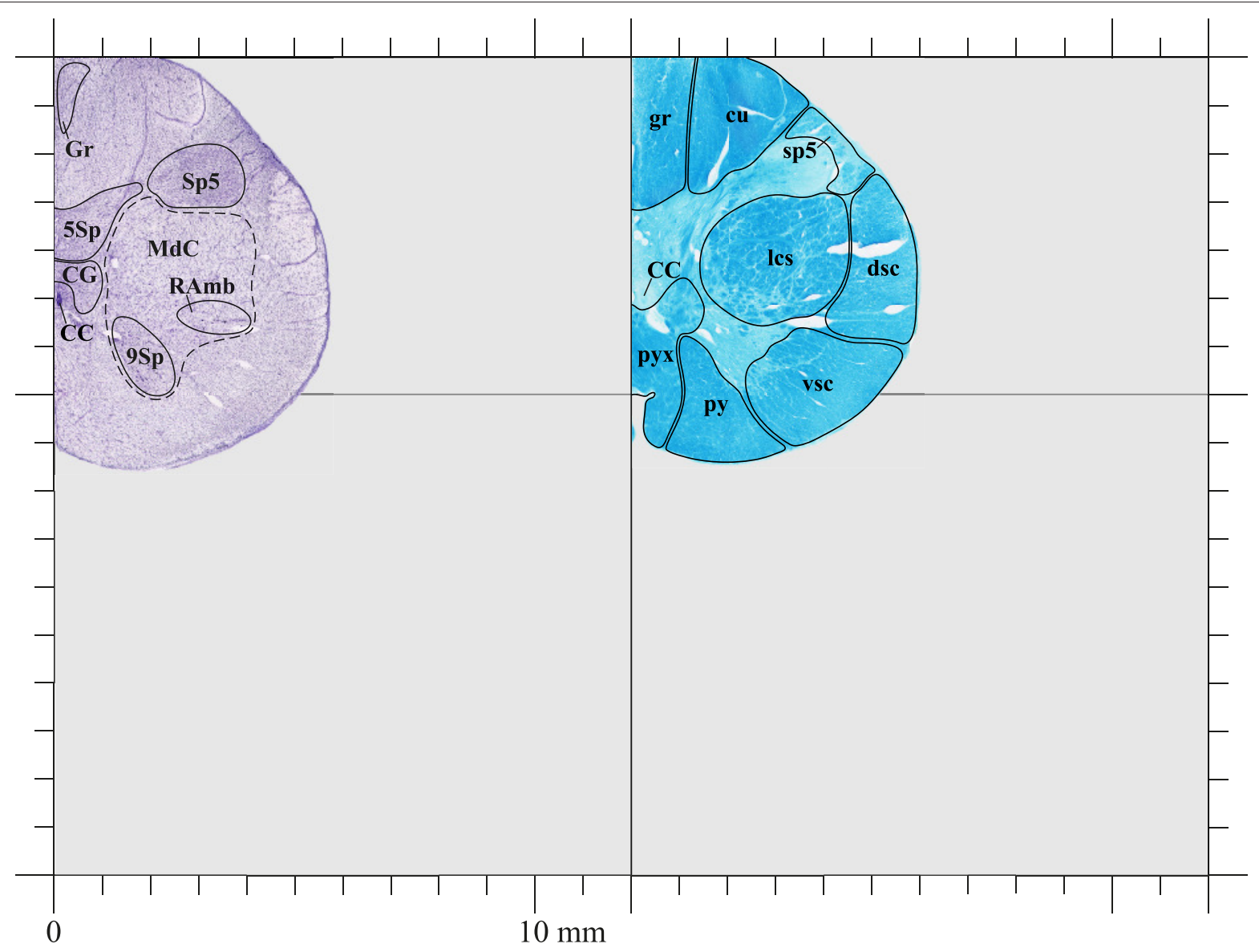

5Sp : Lamina 5 of the spinal gray

9Sp: Lamina 9 of the spinal gray

CC : Central canal

CC : Central canal

CG : Central gray

Gr: Gracile nucleus

MdC : Medullary reticular nucleus, central part

RAmb : Retroambiguus nucleus

Sp5 : $\quad$ Spinal trigeminal nucleus

cu : Cuneate fasciculus

dsc : Dorsal spinocerebellar tract

gr : Gracile fasciculus

lcs : Lateral corticospinal tract

py : Pyramidal tract

pyx : Pyramidal decussation

sp5: $\quad$ Spinal trigeminal tract

vsc : Ventral spinocerebellar tract

\section{Plate 45}

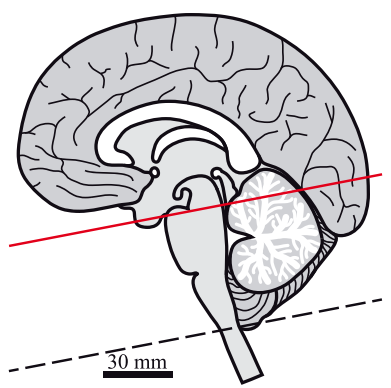

$-51.70 \mathrm{~mm}$ 


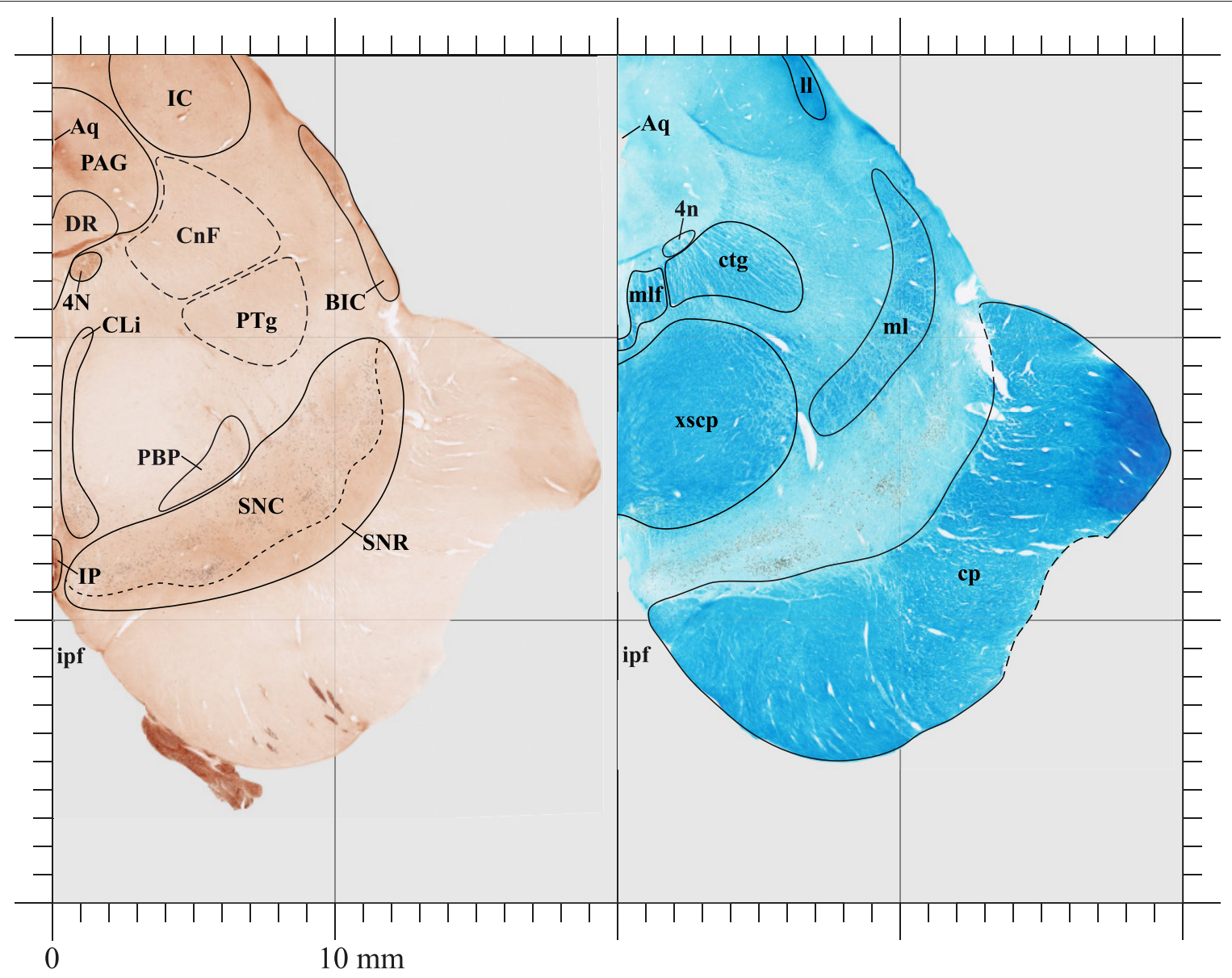

4N: $\quad$ Trochlear nucleus

Aq : Aqueduct

BIC : Nucleus of the brachium of the inferior colliculus

CLi : Caudal linear nucleus of the raphe

$\mathrm{CnF}$ : Cuneiform nucleus

DR : Dorsal raphe nucleus

IC : Inferior colliculus

IP : Interpeduncular nucleus

ipf : Interpeduncular fossa

PAG : Periaqueductal gray

PBP : Parabrachial pigmented nucleus of the ventral tegmental area

PTg : Pedunculotegmental nucleus

SNC : Substantia nigra, compact part

SNR : Substantia nigra, reticular part
Plate A

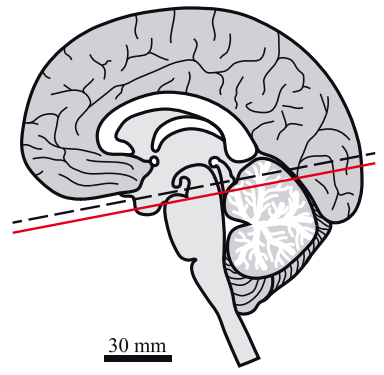

4n: Trochlear nerve

Aq : Aqueduct

cp : Cerebral peduncle

ctg : Central tegmental tract

ipf : Interpeduncular fossa

11: Lateral lemniscus

$\mathrm{ml}$ : $\quad$ Medial lemniscus

mlf : Medial longitudinal fasciculus

xscp : Decussation of the superior cerebellar peduncle 


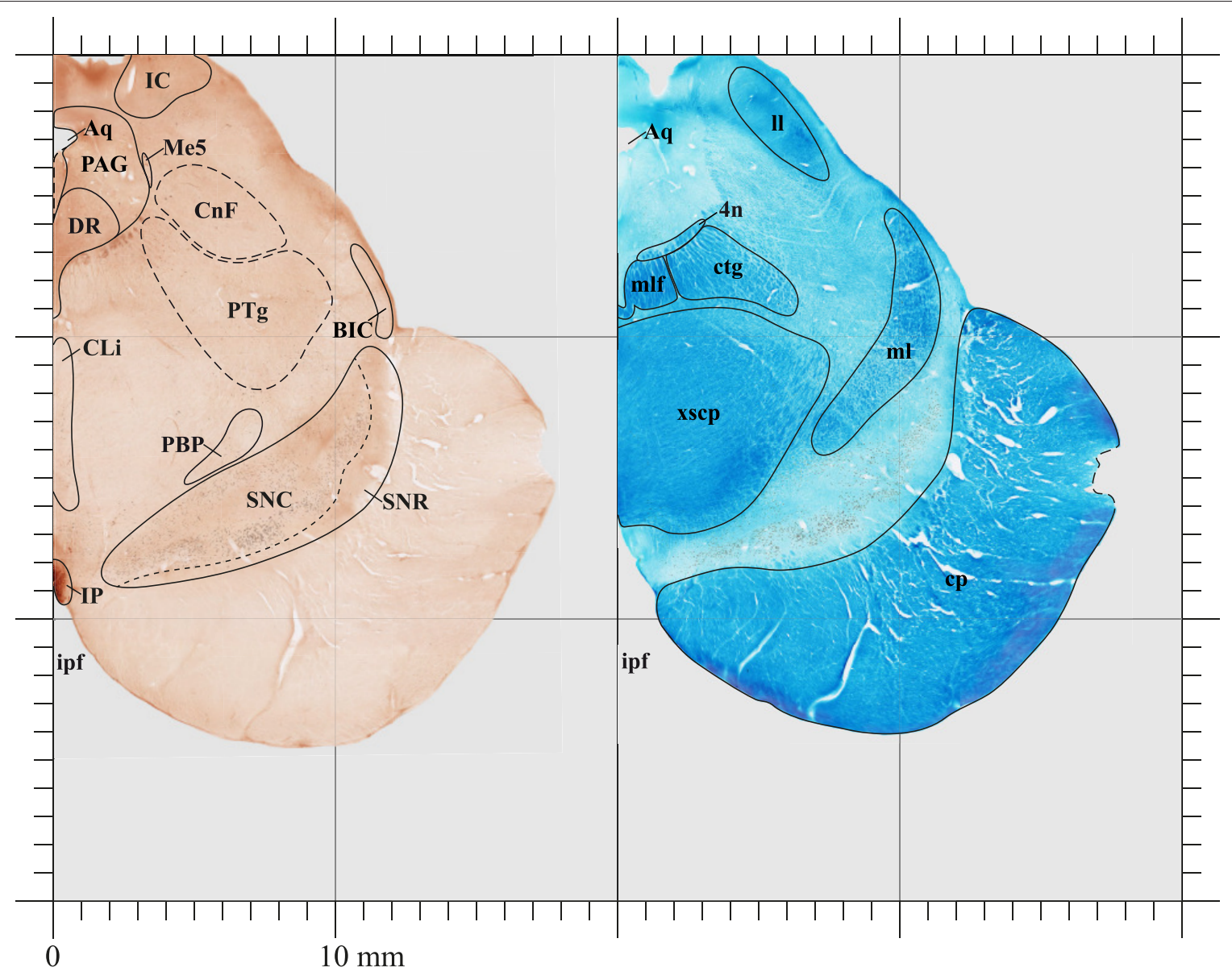

Aq : Aqueduct

BIC : Nucleus of the brachium of the inferior colliculus

CLi : Caudal linear nucleus of the raphe

$\mathrm{CnF}$ : Cuneiform nucleus

DR : Dorsal raphe nucleus

IC : Inferior colliculus

IP : Interpeduncular nucleus

ipf : Interpeduncular fossa

Me5: Mesencephalic trigeminal nucleus

PAG : Periaqueductal gray

PBP : Parabrachial pigmented nucleus of the ventral tegmental area

PTg: Pedunculotegmental nucleus

SNC : Substantia nigra, compact part

SNR : Substantia nigra, reticular part
Plate B

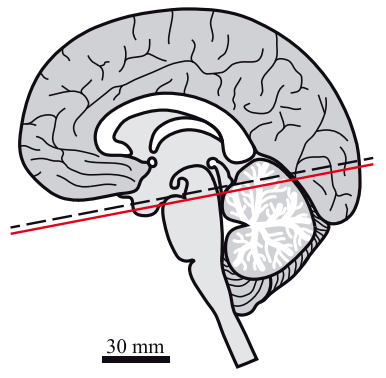

4n: Trochlear nerve

Aq : Aqueduct

cp : $\quad$ Cerebral peduncle

ctg : Central tegmental tract

ipf : Interpeduncular fossa

11: Lateral lemniscus

$\mathrm{ml}$ : $\quad$ Medial lemniscus

mlf: Medial longitudinal fasciculus

xscp : Decussation of the superior cerebellar peduncle 


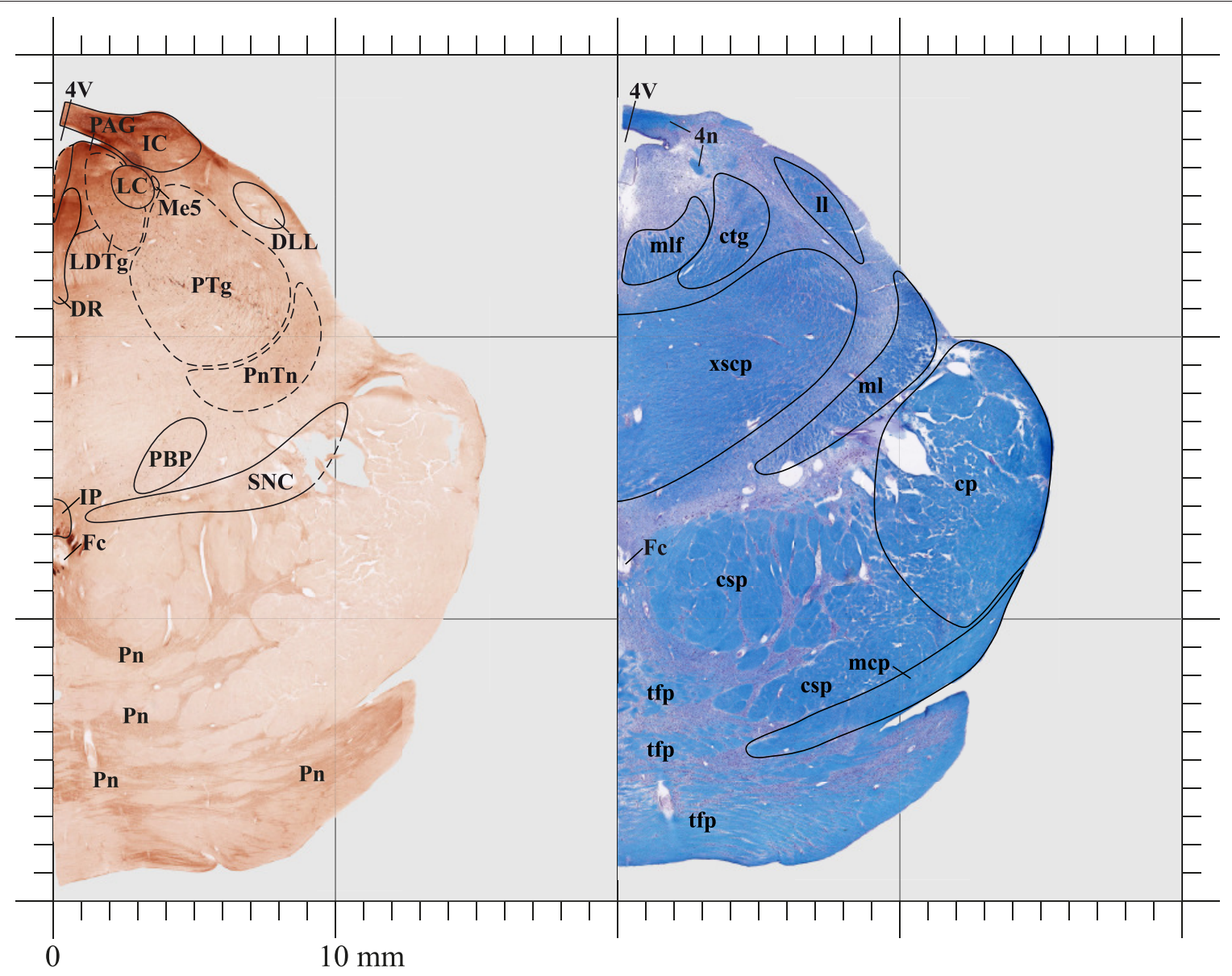

4V : Fourth ventricle

DLL: Dorsal nucleus of the lateral lemniscus

4n: Trochlear nerve

DR : Dorsal raphe nucleus

4V : $\quad$ Fourth ventricle

Fc : Foramen caecum

IC : Inferior colliculus

IP : Interpeduncular nucleus

LC : Locus coeruleus

LDTg : Laterodorsal tegmental nucleus

Me5: Mesencephalic trigeminal nucleus

PAG : Periaqueductal gray

PBP : Parabrachial pigmented nucleus of the ventral tegmental area

cp : Cerebral peduncle

csp : Corticospinal tract

ctg : Central tegmental tract

Fc : Foramen caecum

11: Lateral lemniscus

mcp : Middle cerebellar peduncle

$\mathrm{ml}$ : Medial lemniscus

mlf: Medial longitudinal fasciculus

tfp : $\quad$ Transverse fibers of the pons

Pn : $\quad$ Pontine nuclei

xscp : Decussation of the superior cerebellar peduncle

PnTn : Pontine reticular nucleus, tegmental part

PTg : Pedunculotegmental nucleus

SNC : Substantia nigra, compact part

\section{Plate C}

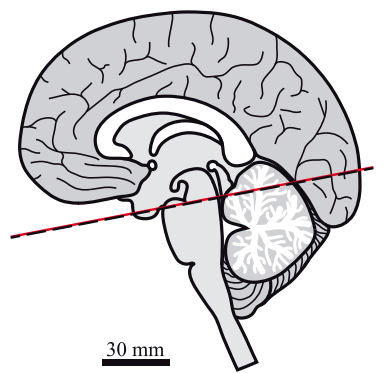

$-0.40 \mathrm{~mm}$ 


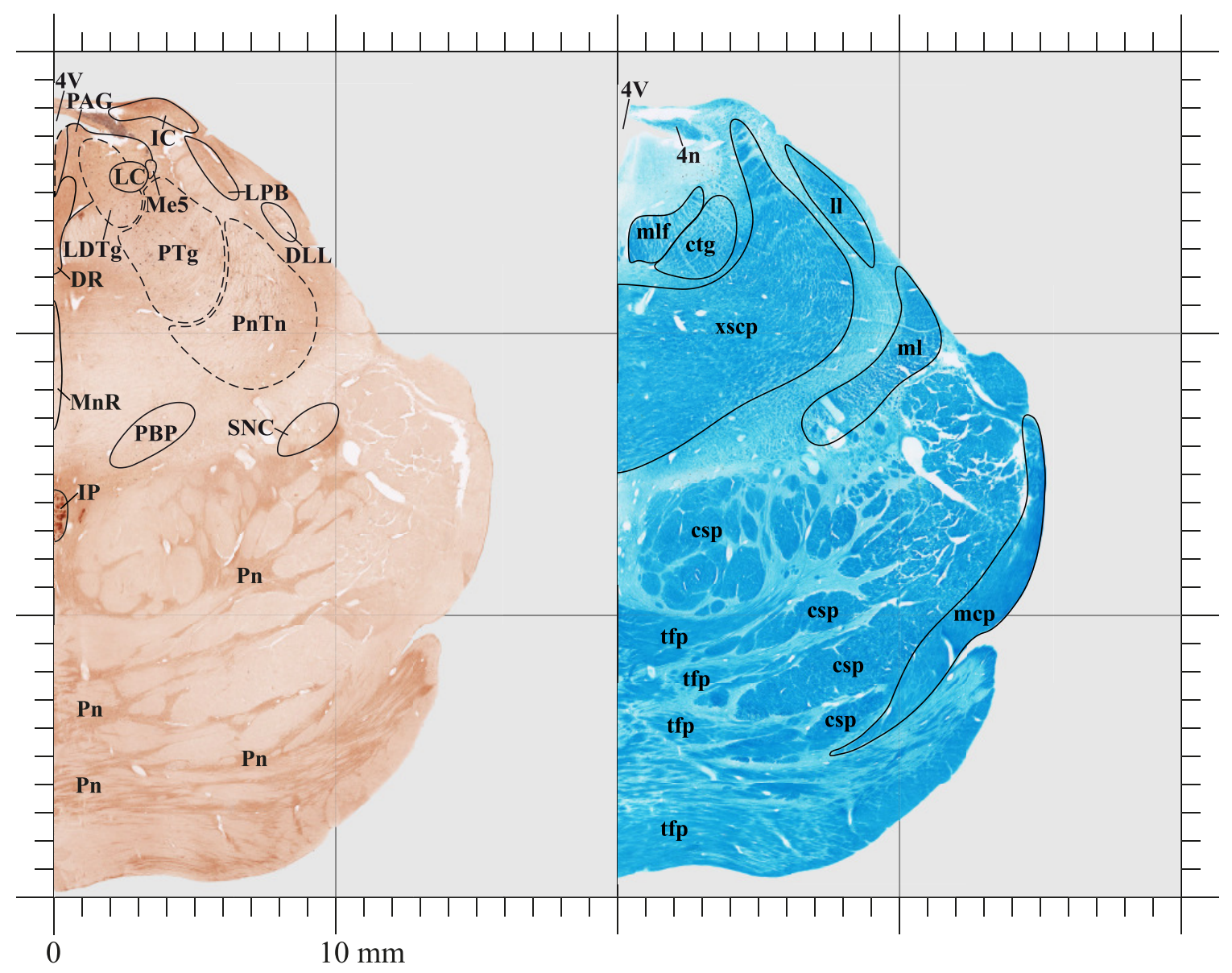

4V: $\quad$ Fourth ventricle

DLL : Dorsal nucleus of the lateral lemniscus

DR : Dorsal raphe nucleus

IC : Inferior colliculus

IP : Interpeduncular nucleus

LC : Locus coeruleus

LDTg : Laterodorsal tegmental nucleus

LPB : Lateral parabrachial nucleus

Me5: Mesencephalic trigeminal nucleus

MnR : Median raphe nucleus

PAG : Periaqueductal gray

PBP : Parabrachial pigmented nucleus of the ventral tegmental area

Pn : $\quad$ Pontine nuclei

PnTn : Pontine reticular nucleus, tegmental part

PTg: Pedunculotegmental nucleus

SNC : Substantia nigra, compact part
Plate D

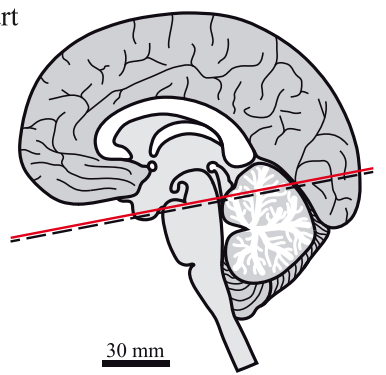

4n: Trochlear nerve

4V: $\quad$ Fourth ventricle

csp : Corticospinal tract

ctg : Central tegmental tract

11: $\quad$ Lateral lemniscus

mcp : Middle cerebellar peduncle

$\mathrm{ml}$ : $\quad$ Medial lemniscus

mlf : Medial longitudinal fasciculus

tfp : Transverse fibers of the pons

xscp : Decussation of the superior cerebellar peduncle 


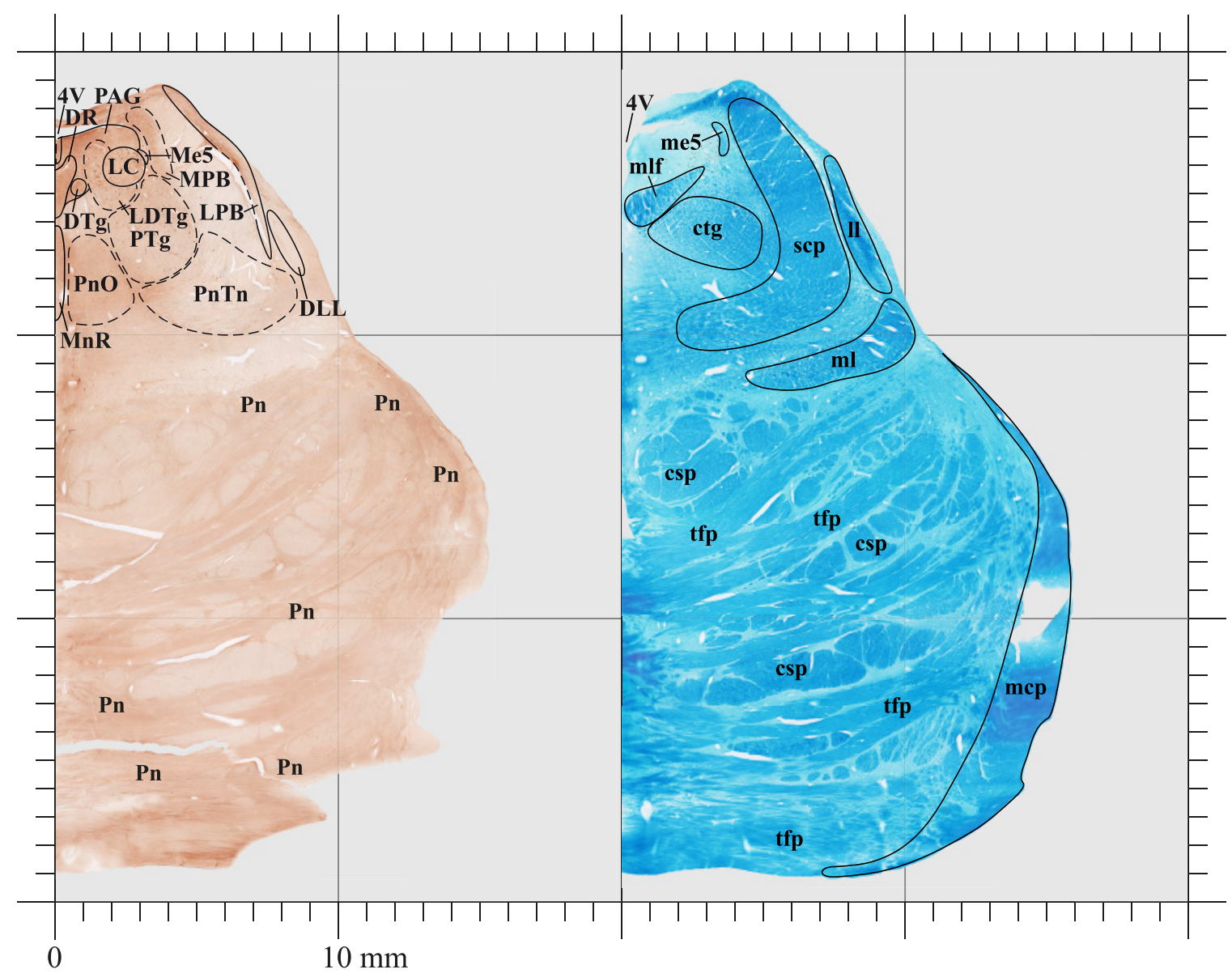

4V: $\quad$ Fourth ventricle

DLL : Dorsal nucleus of the lateral lemniscus

DR : Dorsal raphe nucleus

DTg : Dorsal tegmental nucleus

LC : Locus coeruleus

LDTg : Laterodorsal tegmental nucleus

LPB : Lateral parabrachial nucleus

Me5: Mesencephalic trigeminal nucleus

MnR : Median raphe nucleus

MPB : Medial parabrachial nucleus

PAG : Periaqueductal gray

Pn : $\quad$ Pontine nuclei

PnO : Pontine reticular nucleus, oral part

PnTn: Pontine reticular nucleus, tegmental part

PTg : Pedunculotegmental nucleus
Plate E
4V: $\quad$ Fourth ventricle

csp : Corticospinal tract

ctg : Central tegmental tract

11: Lateral lemniscus

mcp : Middle cerebellar peduncle

me5 : Mesencephalic trigeminal tract

$\mathrm{ml}$ : $\quad$ Medial lemniscus

mlf: Medial longitudinal fasciculus

scp : $\quad$ Superior cerebellar peduncle

tfp : $\quad$ Transverse fibers of the pons

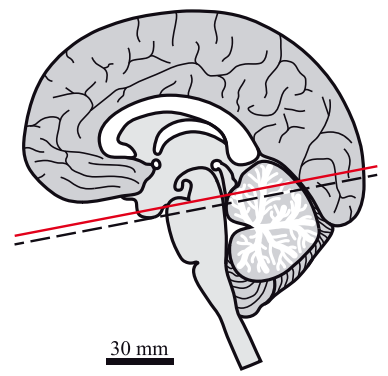

$-3.77 \mathrm{~mm}$ 


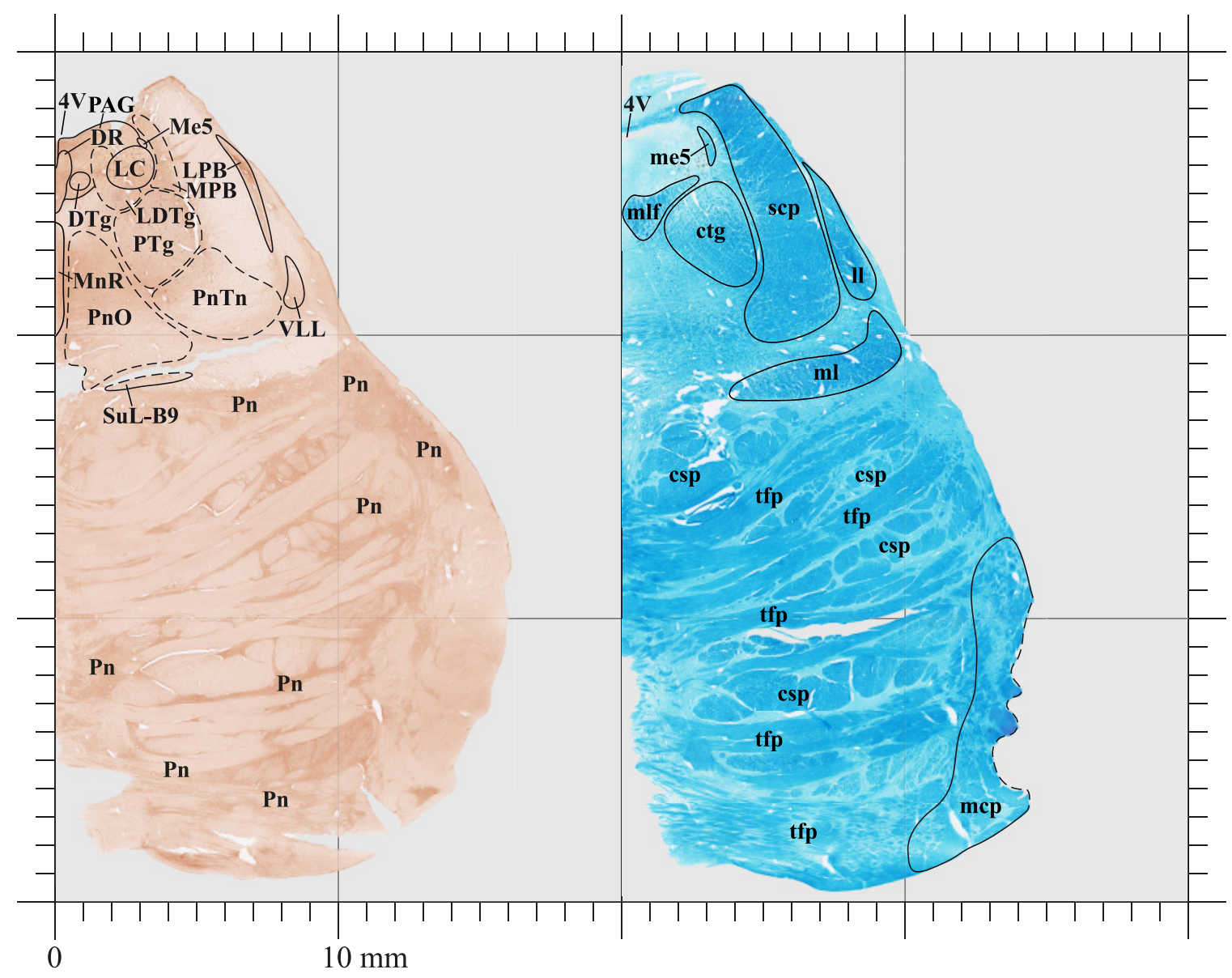

4V : $\quad$ Fourth ventricle

DR: Dorsal raphe nucleus

DTg : Dorsal tegmental nucleus

LC : Locus coeruleus

LDTg: Laterodorsal tegmental nucleus

LPB : Lateral parabrachial nucleus

Me5 : Mesencephalic trigeminal nucleus

MnR : Median raphe nucleus

MPB : Medial parabrachial nucleus

PAG : Periaqueductal gray

Pn : $\quad$ Pontine nuclei

$\mathrm{PnO}$ : Pontine reticular nucleus, oral part

PnTn : Pontine reticular nucleus, tegmental part

PTg: Pedunculotegmental nucleus

SuL-B9 : Supralemniscal nucleus - B9 serotonin cells

VLL: Ventral nucleus of the lateral lemniscus

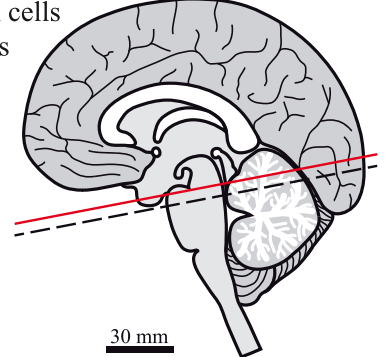

\section{Plate F}

4V: $\quad$ Fourth ventricle

csp : Corticospinal tract

ctg : Central tegmental tract

11: Lateral lemniscus

mcp : Middle cerebellar peduncle

me5 : Mesencephalic trigeminal tract

$\mathrm{ml}$ : $\quad$ Medial lemniscus

mlf: Medial longitudinal fasciculus

scp : $\quad$ Superior cerebellar peduncle

tfp : $\quad$ Transverse fibers of the pons 


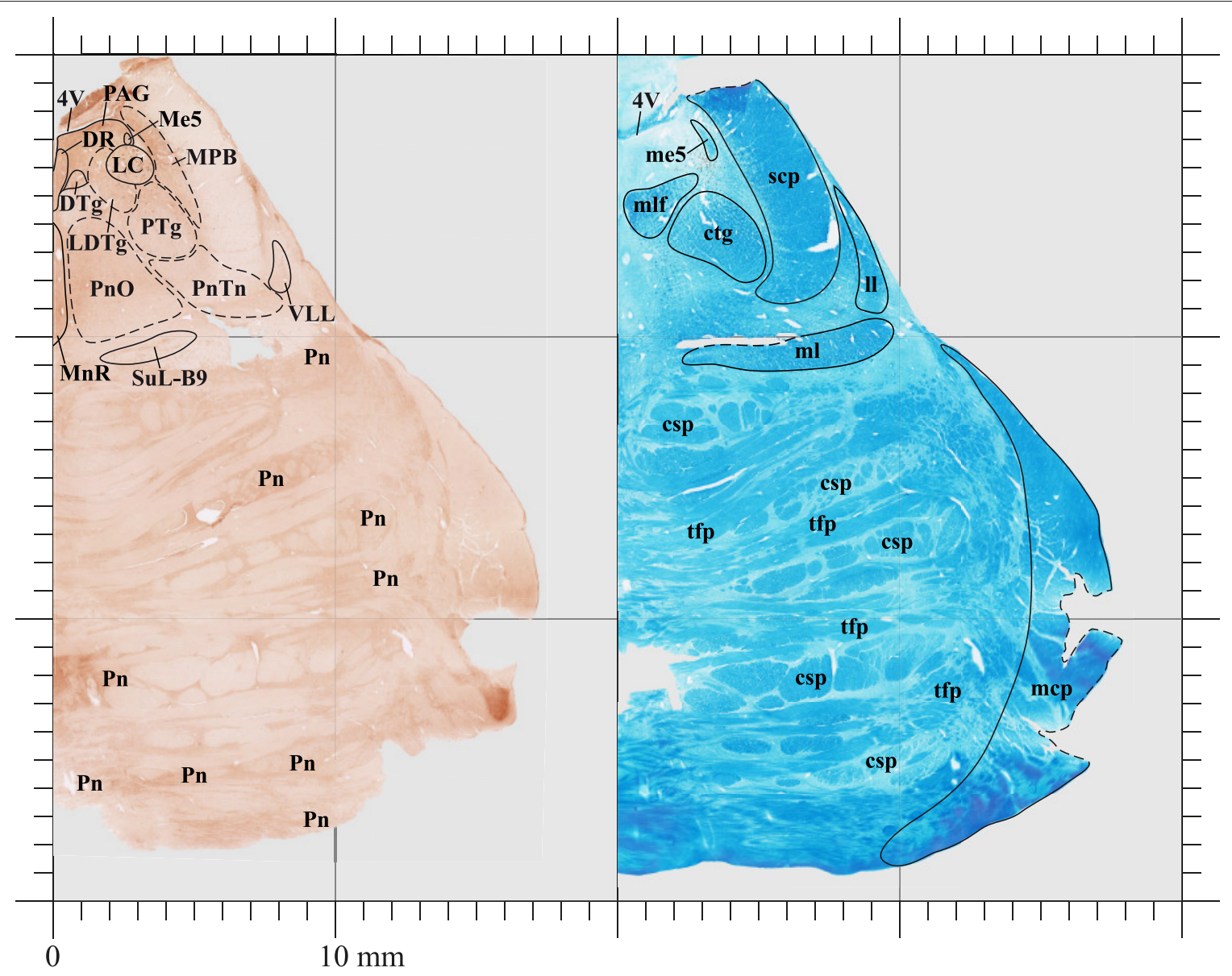

4V : Fourth ventricle

DR: Dorsal raphe nucleus

DTg : Dorsal tegmental nucleus

LC : Locus coeruleus

LDTg : Laterodorsal tegmental nucleus

Me5: Mesencephalic trigeminal nucleus

MnR: Median raphe nucleus

MPB : Medial parabrachial nucleus

PAG : Periaqueductal gray

Pn : $\quad$ Pontine nuclei

PnO : Pontine reticular nucleus, oral part

PnTn : Pontine reticular nucleus, tegmental part

PTg: Pedunculotegmental nucleus

SuL-B9 : Supralemniscal nucleus - B9 serotonin cells

VLL: Ventral nucleus of the lateral lemniscus

\section{Plate G}

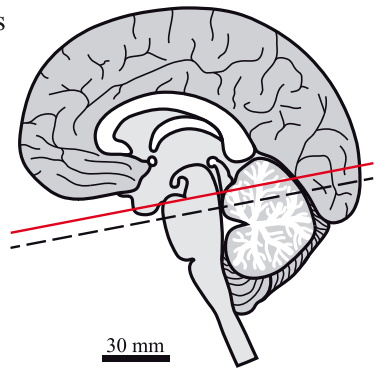

4V: $\quad$ Fourth ventricle

csp : Corticospinal tract

ctg : Central tegmental tract

11: Lateral lemniscus

mcp : Middle cerebellar peduncle

me5 : Mesencephalic trigeminal tract

$\mathrm{ml}$ : $\quad$ Medial lemniscus

mlf: Medial longitudinal fasciculus

scp : $\quad$ Superior cerebellar peduncle

tfp : $\quad$ Transverse fibers of the pons 


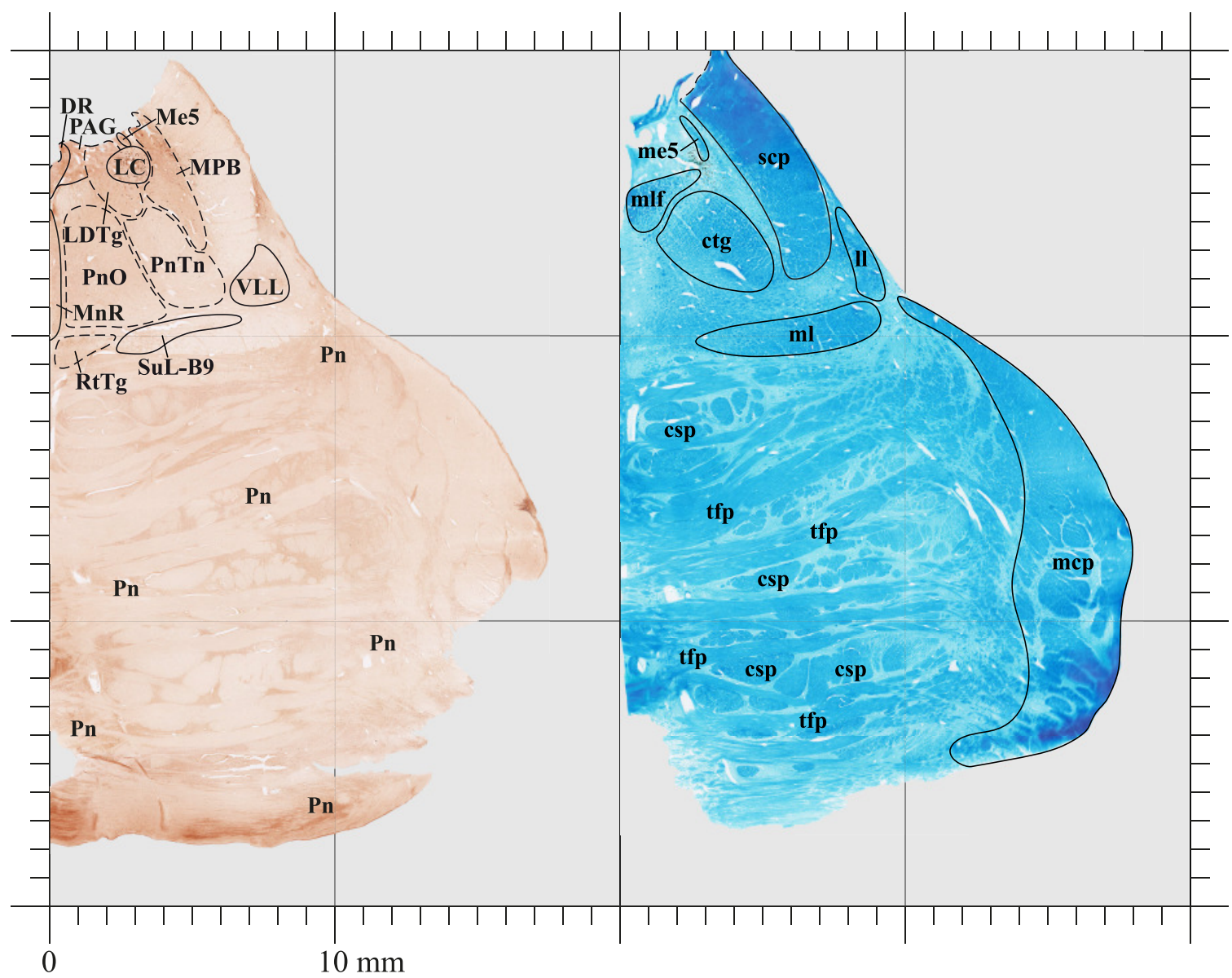

DR : Dorsal raphe nucleus

csp : Corticospinal tract

LC : Locus coeruleus

ctg : Central tegmental tract

LDTg: Laterodorsal tegmental nucleus

Me5: Mesencephalic trigeminal nucleus

11: Lateral lemniscus

MnR: Median raphe nucleus

mcp : Middle cerebellar peduncle

MPB : Medial parabrachial nucleus

me5: Mesencephalic trigeminal tract

PAG : Periaqueductal gray

Pn : $\quad$ Pontine nuclei

$\mathrm{ml}$ : Medial lemniscus

$\mathrm{PnO}$ : $\quad$ Pontine reticular nucleus, oral part

mlf: Medial longitudinal fasciculus

scp : $\quad$ Superior cerebellar peduncle

PnTn : Pontine reticular nucleus, tegmental part

tfp : $\quad$ Transverse fibers of the pons

RtTg: Reticulotegmental nucleus

SuL-B9 : Supralemniscal nucleus - B9 serotonin cells

VLL : Ventral nucleus of the lateral lemniscus

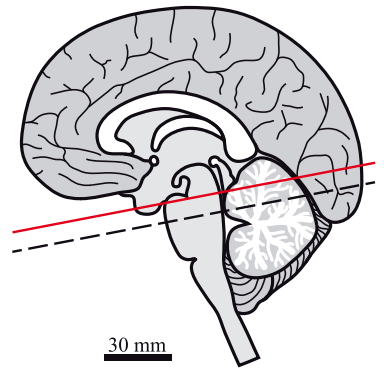




\section{DATA AVAILABILITY STATEMENT}

The original contributions presented in the study are included in the article/Supplementary Material, further inquiries can be directed to the corresponding author.

\section{ETHICS STATEMENT}

The studies involving human participants were reviewed and approved by Ethics Committee at Universite Laval. The patients/participants provided their written informed consent to participate in this study.

\section{AUTHOR CONTRIBUTIONS}

MT and ÉP provided post-mortem human brain tissue. LG and MP were in charge of MRI and proceeded with brain dissection. MT, LG, and MP perfused and extracted the brain. LG cut the brainstem and stained sections with Cresyl Violet and Luxol Fast Blue. VC stained sections with ChAT and acquired and edited images. SS, AP, and MP were in charge of brainstem nuclei and fiber tracts segmentation. VC, AP, and MP wrote the

\section{REFERENCES}

Afshar, F., Watkins, E. S., and Yap, J. C. (1978). Stereotaxic Atlas of the Human Brainstem and Cerebellar Nuclei. New York, NY: Raven Press.

Bianciardi, M., Toschi, N., Edlow, B. L., Eichner, C., Setsompop, K., Polimeni, J. R., et al. (2015). Toward an in vivo neuroimaging template of human brainstem nuclei of the ascending arousal, autonomic, and motor systems. Brain Connect. 5, 597-607. doi: 10.1089/brain.2015.0347

Deistung, A., Schafer, A., Schweser, F., Biedermann, U., Gullmar, D., Trampel, R., et al. (2013). High-resolution MR imaging of the human brainstem in vivo at 7 tesla. Front. Hum. Neurosci. 7:710. doi: 10.3389/fnhum.2013.00710

Ding, S. L., Royall, J. J., Sunkin, S. M., Ng, L., Facer, B. A., Lesnar, P., et al. (2016). Comprehensive cellular-resolution atlas of the adult human brain. J. Comp. Neurol. 524, 3127-3481. doi: 10.1002/cne.24080

Erbagci, H., Keser, M., Kervancioglu, S., and Kizilkan, N. (2012). Estimation of the brain stem volume by stereological method on magnetic resonance imaging. Surg. Radiol. Anat. 34, 819-824. doi: 10.1007/s00276-012-0966-3

Ferraye, M. U., Debu, B., and Pollak, P. (2010). Deep brain stimulation and gait disorders in Parkinson disease. Rev. Neurol. 166, 178-187. doi: 10.1016/j.neurol.2009.07.018

Goetz, L., Bhattacharjee, M., Ferraye, M. U., Fraix, V., Maineri, C., Nosko, D., et al. (2019). Deep brain stimulation of the pedunculopontine nucleus area in Parkinson disease: MRI-based anatomoclinical correlations and optimal target. Neurosurgery 84, 506-518. doi: 10.1093/neuros/nyy151

Insola, A., Valeriani, M., and Mazzone, P. (2012). Targeting the pedunculopontine nucleus: a new neurophysiological method based on somatosensory evoked potentials to calculate the distance of the deep brain stimulation lead from the Obex. Neurosurgery 71, 96-103. doi: 10.1227/NEU.0b013e318249c726

Jacobsohn, L. (1909). Über die Kerne des Menschlichen Hirnstamms (Medulla oblongata, Pons, und Pedunculus cerebri). Berlin: Verlag der Konigl Akademie der Wissenschaften.

Kolliker, A. (1896). Handbuch der Gewebelehre des Menschen. Leipzig: Engelmann.

Naidich, T. P., Duvernoy, H. M., Delman, B. M., Sorensen, A. G., Kollias, S. S., and Haacke, E. M. (2007). Duvernoy's Atlas of the Human Brain Stem and Cerebellum. Wien: Springer Verlag.

Olszewski, J., and Baxter, D. (1954). Cytoarchitecture of the Human Brain Stem. Basel: J.B. Lippincott Company. manuscript. All authors contributed to the article and approved the submitted version.

\section{FUNDING}

This work was supported by research grants from the Quebec Bio-Imaging Network (QBIN) and the Natural Sciences and Engineering Research Council of Canada (NSERC) to MP who benefited from a senior career award from the Fonds de Recherche du Québec-Santé (FRQ-S). VC was the recipient of MSc and BSc fellowships from FRQ-S and NSERC.

\section{ACKNOWLEDGMENTS}

The authors are very grateful for brain donation. The authors thank Marie-Josée Wallman and Sylvain Courcy (IRM Québec) for technical assistance.

\section{SUPPLEMENTARY MATERIAL}

The Supplementary Material for this article can be found online: https://www.dropbox.com/sh/ln9zqv4dkygol2q/ AAC80leqF0bYcI8ZLWERCIRXa?dl=0

Parent, A. (1996). Carpenter's Human Neuroanatomy. Baltimore, MD: Williams and Wilkins.

Paxinos, G., Furlong, T. M., and Watson, C. (2020). Human Brainstem: Cytoarchitecture, Chemoarchitecture, Myeloarchitecture, Revised Edition. San Diego, CA: Academic Press.

Paxinos, G., and Huang, X. F. (1995). Atlas of the Human Brainstem. San Diego, CA: Academic Press.

Paxinos, G., and Watson, C. (1982). The Rat Brain in Stereotaxic Coordinates Sydney, NSW: Academic Press.

Riley, H. A. (1943). An Atlas of the Basal Ganglia, Brain Stem and Spinal Cord. New York, NY: Hafner Publishing Company.

Rushmore, R. J., Wilson-Braun, P., Papadimitriou, G., Ng, I., Rathi, Y., Zhang, F., et al. (2020). 3D Exploration of the brainstem in 50micron resolution MRI. Front. Neuroanat. 14:40. doi: 10.3389/fnana.2020. 00040

Schaltenbrand, G., and Wahren, W. (1977). Atlas for Stereotaxy of the Human Brain. Chicago, IL: Thieme.

Spetzler, R. F., Kalani, M. Y. S., Nakaji, P., and Yagmurlu, K. (2017). Color Atlas of Brainstem Surgery. New York, NY: Thieme.

Talairach, J., David, M., Tournoux, P., Corredor, H., and Kvasina, T. (1957). Atlas d'anatomie stéréotaxique. Repérage radiologique indirect des noyaux gris centraux des régions mésencephalo-sousoptique et hypothalamique de l'homme. Paris: Masson and Cie.

Talairach, J., and Szikla, G. (1967). Atlas of Stereotaxic Anatomy of the Telencephalon. Anatomo-Radiological Studies. Paris: Masson and Cie.

Talairach, J., and Tournoux, P. (1988). Co-Planar Stereotactic Atlas of the Human Brain. 3-Dimensional Proportional System: An Approach to Cerebral Imaging. Stuttgart: Georg Thieme Verlag.

Tang, Y., Sun, W., Toga, A. W., Ringman, J. M., and Shi, Y. (2018). A probabilistic atlas of human brainstem pathways based on connectome imaging data. Neuroimage 169, 227-239. doi: 10.1016/j.neuroimage.2017. 12.042

Terminology, F. C. O. A. (1998). Terminologia Anatomica: International Anatomical Terminology. New York, NY: Thieme Stuttgart.

Thevathasan, W., Coyne, T. J., Hyam, J. A., Kerr, G., Jenkinson, N., Aziz, T. Z., et al. (2011). Pedunculopontine nucleus stimulation improves gait 
freezing in Parkinson disease. Neurosurgery 69, 1248-1253. discussion 1254. doi: 10.1227/NEU.0b013e31822b6f71

Thevathasan, W., Pogosyan, A., Hyam, J. A., Jenkinson, N., Foltynie, T., Limousin, P., et al. (2012). Alpha oscillations in the pedunculopontine nucleus correlate with gait performance in parkinsonism. Brain 135, 148-160. doi: 10.1093/brain/awr315

Watson, C., Bartholomaeus, C., and Puelles, L. (2019). Time for radical changes in brain stem nomenclature-applying the lessons from developmental gene patterns. Front. Neuroanat. 13:10. doi: 10.3389/fnana.2019.00010

Willis, T. (1664). Cerebri Anatome: Cui Accessit Nervorum Descriptio et Usus. London: Martyn and Allestry.

Ziehen, G. T. (1903). Makroskopische und mikroskopische Anatomie des Gehirn. Jena: Fisher.

Zrinzo, L., Zrinzo, L. V., Tisch, S., Limousin, P. D., Yousry, T. A., Afshar, F., et al. (2008). Stereotactic localization of the human pedunculopontine nucleus: atlas-based coordinates and validation of a magnetic resonance imaging protocol for direct localization. Brain 131, 1588-1598. doi: 10.1093/brain/awn075
Conflict of Interest: The authors declare that the research was conducted in the absence of any commercial or financial relationships that could be construed as a potential conflict of interest.

Publisher's Note: All claims expressed in this article are solely those of the authors and do not necessarily represent those of their affiliated organizations, or those of the publisher, the editors and the reviewers. Any product that may be evaluated in this article, or claim that may be made by its manufacturer, is not guaranteed or endorsed by the publisher.

Copyright $\odot 2021$ Coulombe, Saikali, Goetz, Takech, Philippe, Parent and Parent. This is an open-access article distributed under the terms of the Creative Commons Attribution License (CC BY). The use, distribution or reproduction in other forums is permitted, provided the original author(s) and the copyright owner(s) are credited and that the original publication in this journal is cited, in accordance with accepted academic practice. No use, distribution or reproduction is permitted which does not comply with these terms. 